\title{
Permutation Spreading Technique Employing Spatial Modulation For MIMO-CDMA Systems
}

\author{
Nordine Quadar
}

Thesis submitted in partial fulfillment of the requirements

For the Master of Applied Science degree in

Electrical and Computer Engineering

School of Electrical Engineering and Computer Science

Faculty of Engineering

University of Ottawa

(c) Nordine Quadar, Ottawa, Canada, 2017 
In the name of Allah, the Most Gracious and the Most Merciful 


\begin{abstract}
Spatial Modulation (SM) is a spatial multiplexing technique designed for MIMO systems where only one transmit antenna is used at each time. It is considered to be an attractive choice for future wireless communication systems as it reduces Inter-Channel Interference (ICI) while maintaining high energy efficiency. It can achieve this goal by mapping block of data bits into constellation points in the spatial and signal domain. Combining this innovative method with multiple access techniques could improve the system performance and enhance the data rate. In Code Division Multiple Access (CDMA) method employing parity bit permutation spreading, the bit error rate (BER) performance could be improved by using the parity bits to select the spreading sequence to use at each signaling interval. In this thesis, a new system model based on SM and CDMA employing parity bit permutation spreading is proposed and investigated. The proposed system takes advantage of the benefits of both techniques.

In this system, in addition to use the parity bits to select the spreading sequences, same concept is used to select the combination of antennas to activate at each time instant. By doing so, a reduction of power consumption, Inter-Channel and Inter Symbol Interference effect can be achieved while keeping a certain diversity order compared to SM. Multiuser scenario is also discussed in order to investigate the multiple access interference (MAI) effects in synchronous transmission. In such case, the receiver estimates the desired user's information by considering the other users' signal as additional noise.

Simulation results of the proposed MIMO-CDMA system employing permutation spreading show, for single user and multiuser, a significant improvement of the BER performance in low signal to noise ratio (SNR) when SM is implemented.
\end{abstract}




\section{Acknowledgements}

First and foremost, I would like to thank Allah Almighty for his blessing and giving me the power to accomplish this milestone.

I would like to express my deepest gratitude and sincere appreciation to my supervisor Dr. Claude D'Amours for his support, guidance, valuable advices and encouragement throughout this research. Without his help, this thesis would not have been achievable. I feel very privileged having such a mentor.

Last but not least, I would like to thank my beloved wife Manal for her love, patience and continued support. Special thanks go to my mother for her prayers, blessings and unconditional love. I am also grateful to all my family members for their support. 
To the loving memory of my father, To my soon-to-be-born baby. 


\section{Table of Contents}

List of Tables $\quad$ ix

List of Figures $\quad$ X

List of Abbreviations $\quad$ xiii

List of Symbols $\quad$ xvi

1 Introduction $\quad 1$

1.1 Motivation and background ...................... 1

1.2 Thesis scope . . . . . . . . . . . . . . . . . . . 4

1.3 Thesis objectives . . . . . . . . . . . . . . . . . . 5

1.4 Scientific methods employed . . . . . . . . . . . . . . . . 6

1.5 Thesis contributions ......................... 6

1.6 Thesis organisation . . . . . . . . . . . . . . . . . 7

2 Permutation Spreading For MIMO-CDMA System $\quad 9$

2.1 Introduction . . . . . . . . . . . . . . . . . . 9

2.2 System Model . . . . . . . . . . . . . . . . . . . 10

2.2.1 Transmitter Model . . . . . . . . . . . . . . . . . . . . . 10 
$2.2 .2 \quad$ Receiver Model . . . . . . . . . . . . . . . . . . . . . . . . . . 14

2.3 Theoretical analysis of the BER performance . . . . . . . . . . . . 19

2.3.1 BER analysis for MIMO-CDMA system with parity bit selected

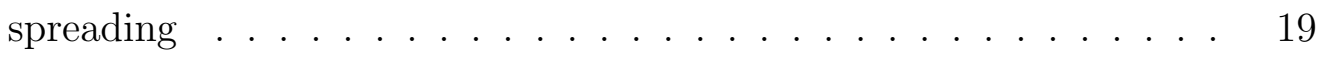

2.3.2 BER analysis for MIMO-CDMA system with Permutation Spreading 20

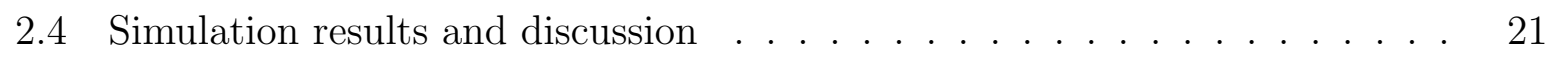

2.4 .1 Discussion . . . . . . . . . . . . . . . . . . 25

3 Spatial Modulation $\quad 26$

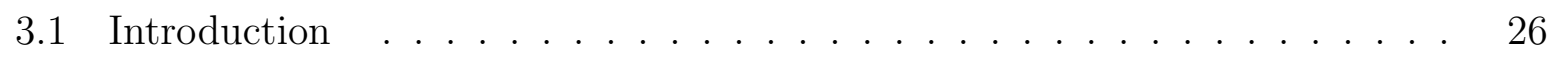

3.2 Spatial Modulation $(\mathrm{SM})$ system model . . . . . . . . . . . . . . . 28

3.2.1 Transmitter Model . . . . . . . . . . . . . . . . . . . . . . . . 28

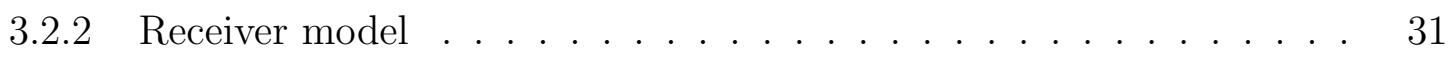

3.3 Generalized Spatial Modulation (GSM) system model . . . . . . . . . . . . 33

3.3 .1 Transmitter Model . . . . . . . . . . . . . . . . . . . 33

$3.3 .2 \quad$ Receiver Model . . . . . . . . . . . . . . . . . . . . . 35

3.4 Receiver complexity . . . . . . . . . . . . . . . . . . . 36

3.5 Simulation results and discussion $\ldots \ldots \ldots \ldots$

3.5 .1 Discussion . . . . . . . . . . . . . . . . . . . . 41

4 Single User Permutation Spreading Employing Spatial Modulation for MIMO-CDMA system $\quad 42$

4.1 Introduction . . . . . . . . . . . . . . . . . . . . . . 42

4.2 System Model . . . . . . . . . . . . . . . . . . . . . . . . . . 43

4.2 .1 Transmitter Model . . . . . . . . . . . . . . . . . . . 43

$4.2 .2 \quad$ Receiver Model . . . . . . . . . . . . . . . . . . 47 
4.3 Theoretical analysis of the BER performance . . . . . . . . . . . . 52

4.3 .1 MISO-CDMA system . . . . . . . . . . . . . . . . 52

4.3.2 MIMO-CDMA system .................. 56

4.4 Simulation results and discussion . . . . . . . . . . . . . 57

4.4.1 Theoretical and Simulation results of MIMO/CDMA with SM design 57

4.4.2 Comparison of simulation results . . . . . . . . . . . 58

4.4 Discussion ......................... 63

5 Multiuser Permutation Spreading Employing Spatial Modulation For $\begin{array}{ll}\text { Synchronous MIMO-CDMA systems } & 64\end{array}$

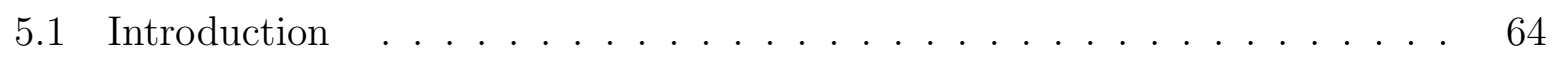

5.2 System Model . . . . . . . . . . . . . . . . . 65

5.2.1 Transmitter Model . . . . . . . . . . . . . . . 65

5.2.2 Receiver Model . . . . . . . . . . . . . . . . . . . 66

5.3 Simulation results and discussion . . . . . . . . . . . . . . 69

5.3.1 Simulation results comparison . . . . . . . . . . . 69

5.3 .2 Discussion ......................... 71

6 Conclusion and suggestion for future work $\quad 73$

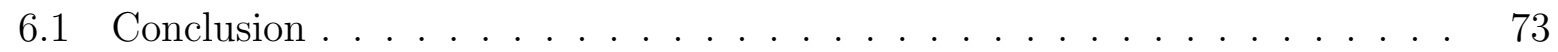

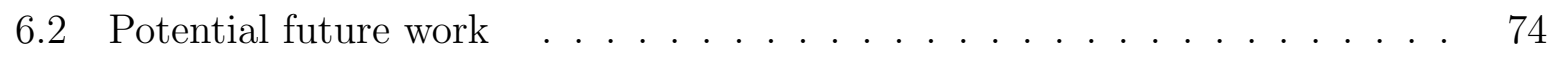

$\begin{array}{ll}\text { References } & 76\end{array}$ 


\section{List of Tables}

2.1 T-Design Permutations Spreading for MIMO-CDMA system with 4 transmit

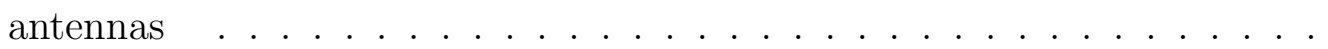

2.2 STBC-Design Permutations Spreading for MIMO-CDMA system with 4 transmit antennas . . . . . . . . . . . . . . . . . . 13

2.3 STBC-Design Permutations Spreading transmit table for 4 transmit antennas 15

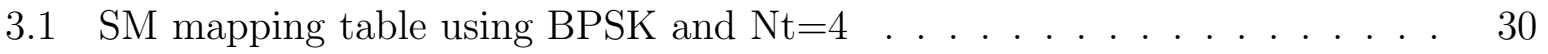

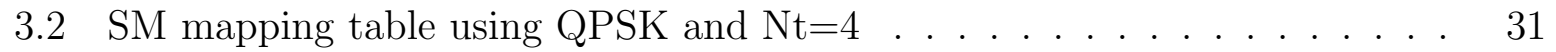

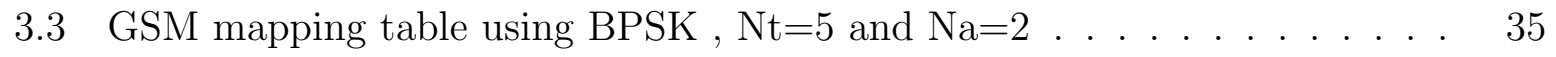

3.4 VGSM mapping table using $\mathrm{Nt}=4 \ldots . . \ldots$. . . . . . . . . . 36

4.1 Permutation Spreading for MIMO-CDMA system with 4 transmit antennas Using STBC design . . . . . . . . . . . . . . . . . . . 45

4.2 SM mapping table for MIMO system with four transmit antennas . . . . . 46

4.3 Permutation Spreading transmit table for 4x4 MIMO-CDMA system using $\mathrm{SM} \ldots \ldots \ldots \ldots \ldots \ldots \ldots \ldots$

4.4 Received vector for MISO system with four transmit antennas and one re-

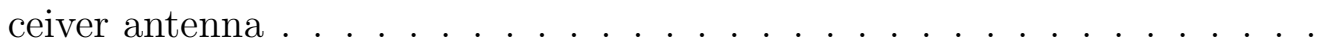




\section{List of Figures}

2.1 Transmitter of MIMO-CDMA with parity bit selected and permutation

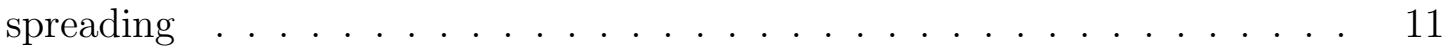

2.2 Receiver of MIMO-CDMA with parity bit selected and permutation spreading 16

2.3 BER performance of MISO-CDMA System with parity bit selected spreading 22

2.4 BER performance of MIMO-CDMA System with parity bit selected spreading 22

2.5 BER performance of MISO-CDMA System with STBC permutation spreading 23

2.6 BER performance of MIMO-CDMA System with STBC permutation spread-

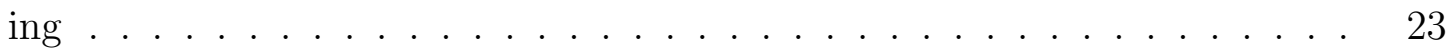

2.7 BER performance comparison of MISO-CDMA System using the three tech-

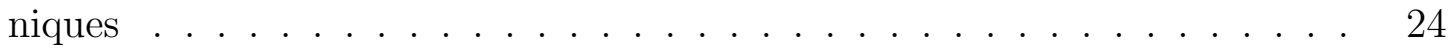

2.8 BER performance comparison of MIMO-CDMA System using the three

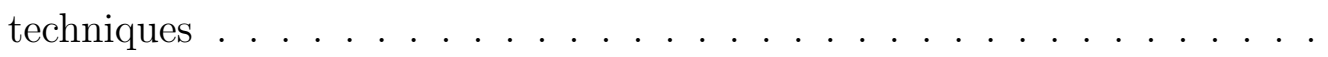

3.1 SM constellation diagram . . . . . . . . . . . . . . . . . 27

3.2 Working concepts of (a) SMX, (b) OSTBC, (c) SM and (d) GSSK . . . . . 29

3.3 Spatial Modulation system model . . . . . . . . . . . . . . . . 30

3.4 Generalized Spatial Modulation system model . . . . . . . . . . . . . . . . 34

3.5 Comparison of receiver complexity between SM and GSM using ML detection 37

3.6 Comparison of SM performances, where $N_{t}=128$ for $\eta=8 b i t s, N_{t}=32$ for $\eta=6$ bits, $N_{t}=8$ for $\eta=4 b i t s$, using BPSK modulation and $N_{r}=4 \ldots$ 
3.7 BER performance of SM, GSM and VGSM where $\eta=4 b i t s, N_{r}=4$ and using BPSK modulation. . . . . . . . . . . . . . . 39

3.8 BER performance of SM, GSM and VGSM where $\eta=6$ bits, $N_{r}=4$ and using BPSK modulation. . . . . . . . . . . . . . . . . . 40

3.9 BER performance of SM, GSM and VGSM where $\eta=$ 8bits, $N_{r}=4$ and using BPSK modulation. . . . . . . . . . . . . . . . . . 40

4.1 The Transmitter of MIMO-CDMA with permutation spreading using SM . 44

4.2 Transmitter for a 4x4 MIMO-CDMA with permutation spreading using SM 46

4.3 Receiver of MIMO/CDMA with permutation spreading using SM . . . . 49

4.4 Theoretical BER performance of 4x1 MISO-CDMA System using Permutation Spreading with conventional STBC design vs SM design . . . . . . . . 59

4.5 Theoretical BER performance of 4x4 MIMO-CDMA System using Permutation Spreading with conventional STBC design vs SM design . . . . . . . 59

4.6 BER performance of MIMO-CDMA System with Permutation Spreading using SM design . . . . . . . . . . . . . . . . . . 60

4.7 BER performance of 4x1 MISO-CDMA System using Permutation Spreading with conventional STBC design vs SM design . . . . . . . . . . . 61

4.8 BER performance of 2x2 MIMO-CDMA System using Permutation Spreading with conventional STBC design vs SM design . . . . . . . . . . . 61

4.9 BER performance of 4x4 MIMO-CDMA System using Permutation Spreading with conventional STBC design vs SM design . . . . . . . . . . 62

4.10 BER performance of MIMO-CDMA System using Permutation Spreading with SM design vs Conventional SM techniques . . . . . . . . . . 62

5.1 Multiuser transmitter of MIMO-CDMA with permutation spreading using $\mathrm{SM} \ldots \ldots \ldots \ldots \ldots \ldots$

5.2 Multiuser receiver of MIMO/CDMA with permutation spreading using SM 68 
5.3 Comparison of BER Performance of 4x4 MISO-CDMA System using SM Permutation Spreading design . . . . . . . . . . . . . . . 71

5.4 Comparison of BER performance of $4 \times 4$ MISO-CDMA System using SM Permutation Spreading design vs STBC design . . . . . . . . . . 72 


\section{List of Abbreviations}

\begin{tabular}{ll} 
AWGN & Additive White Gaussian Noise \\
3GPP & Third Generation Partnership Project \\
BER & Bit Error Rate \\
BLAST & Bell Labs Layered Space Time \\
BPSK & Binary Phase Shift Keying \\
BS & Base Station \\
CDMA & Code Division Multi Access \\
CSI & Channel State Information \\
DS-CDMA & Direct Sequence Code Division Multiple Access \\
DS-SS & Direct-Spread Spread Spectrum \\
FBE-SM & Fractional Bit Encoded Spatial Modulation \\
GPRS & General Packet Radio Service \\
Gbps & Gigabit per second \\
GSM & Generalized Spatial Modulation \\
GSSK & General Space Shift Keying \\
ICI & Inter-Channel Interference \\
IOT & Internet Of Things \\
ISI & Inter-symbol Interference \\
LDS & Low Density Spreading \\
LPMA & Lattice Partition Multiple Access \\
& \\
\hline
\end{tabular}


LLR Log Likelihood Ratio

LTE-A Long-Term Evolution Advanced

MAI Multi-Access Interference

MIMO Multiple-Input Multiple-Output

MISO Multiple-Input Single-Output

ML Maximum Likelihood

MRC Maximal Ratio Combiner

MLD Maximum Likelihood Detection

MUD Multiuser Detection

MUST Multiuser Superposition Transmission

NOMA Non-Orthogonal Multiple Access

OFDM Orthogonal Frequency Division Multiplexing

OMA Orthogonal Multiple Access

OSTBC Orthogonal Space Time Block Coding

pdf Probability Density Function

PDMA Pattern Division Multiple Access

PN Pseudo-Noise

QAM Quadrature Amplitude Modulation

QoS Quality Of Service

QPSK Quadrature Phase Shift Keying

RF Radio-Frequency

SCMA Sparse Code Multiple Access

SD Sphere Decoding

SM Spatial Modulation

SMX Spatial Multiplexing

SNR Signal To Noise Ratio

SS Spread Spectrum

SSK Space Shift Keying

STBC Space Time Block Code

TDMA Time Domain Multiple Access 
UMTS Universal Mobile Telecommunication Service

VGSM Variable Generalized Spatial Modulation

VLS Visible Light Communication

WCDMA Wideband CDMA 


\section{List of Symbols}

$N_{t} \quad$ Number of transmit antennas

$N_{r} \quad$ Number of received antennas

$N_{c u} \quad$ Number of channels

$N_{m} \quad$ Number of transmitted symbols

$s \quad$ Transmitted symbol

$\boldsymbol{Y} \quad$ Received matrix

n Complex Additive White Noise (AWGN)

$\boldsymbol{H}$ Channel gain vector

$\boldsymbol{S} \quad$ Transmitted signal matrix

. Estimated symbol

$p \quad$ Antenna index

\|.\| Absolute-value norm

$\boldsymbol{y}_{\boldsymbol{i}} \quad$ Received vector at the $i t h$ received antenna

$\sigma_{n}^{2} \quad$ Noise variance

$N_{a} \quad$ Number of antenna to activate

(.) The binomial operation

Ł.」 Floor operation

$\rho \quad$ Number of antenna combinations

$\eta \quad$ Spectral efficiency

C Computational Complexity

$R \quad$ complexity ratio 
$w_{i} \quad$ Spreading waveform used by the $i t h$ transmit antenna

$c_{i} \quad$ ith spreading waveform

$M_{i} \quad$ ith coset

$L \quad$ Number of messages per coset

$K \quad$ Number of cosets

$m_{j} \quad$ Bit stream transmitted from the $j t h$ antenna

$E_{b} \quad$ Received energy per bit

$\alpha_{j i} \quad$ Complex channel gain between the $i t h$ transmit antenna and the $j t h$ received antenna

$b_{i}^{(k)} \quad$ The $i t h$ message bits from the $k t h$ message vector

$r_{l}^{k} \quad$ Output of the $l t h$ matched filter at the receive antenna

$U$ Decision variable

$r_{x} \quad$ Received signal

$E_{0} \quad$ Noise spectral density

$M \quad$ Number of cosets

d Euclidean distance

$\hat{\gamma} \quad$ Average received SNR per bit

$d^{q}[i] \quad$ Data bit streams

$Q \quad$ Number of users $q$

$w_{i}^{q} \quad$ Spreading sequences used by the $i t h$ transmit antenna of user $q$

$T \quad$ Bit duration

$p_{n}^{q} \quad$ Pseudo-noise generated code of the $q t h$ user

$x \quad$ Average received energy per bit 


\section{Chapter 1}

\section{Introduction}

\subsection{Motivation and background}

These days, wireless communications is prevalent everywhere in the world and much of our business and entertainment activities are dependent on wireless communication services. The demand for these services continues to grow at a rapid pace and service providers are expected to be able to accommodate a large increase in the number of users with increased data rate requirements. With the development of the Internet of things (IoT), Ericsson has announced that more than 50 billion devices will be connected to the network by 2020 [1]. Therefore, future wireless network should be able to face the tremendous increase of the traffic.

Looking past, wireless communication technologies have witnessed four generations of technology evolution. The first generation system $(1 \mathrm{G})$ was introduced in 1980s and was designed to support voice transmission using analog cellular technology. 1G was replaced by the second generation $(2 \mathrm{G})$ because it had many limitations such as a poor voice quality, low transmission speed and no encryption. The $2 \mathrm{G}$ systems, also known as the Global System for Mobile communications (GSM), started to expand all over the world in 1991. Contrary to $1 \mathrm{G}$, the second generation is designed based on digital cellular technologies, which helped to enhance the spectral efficiency of the communication system. Text messaging service was first introduced in GSM and a later release allowed low speed data 
transmission using General Packet Radio Service (GPRS). The increasing number of 2G users caused an increasing demand for high-speed data access. To face this issue, the third generation $(3 \mathrm{G})$ came to life in 2001 with a service that can support data rate up to $2 \mathrm{Mbps}$ [2]. The evolution of 3G for CDMA (Code Division Multiple Access) led to CDMA2000, which is an improved version of CDMA used in $2 \mathrm{G}$. The second widely used 3G technology is the WCDMA (Wideband CDMA), also called UMTS (Universal Mobile Telecommunication Service). Although the $3 \mathrm{G}$ systems provide high-speed data service, the increasing number of connected devices and the diversity of content to transmit have required higher transmission speed and date rate. Therefore, new generation technology was needed in order to align with the increasing demand. Forth generation $(4 \mathrm{G})$, also called long-term evolution advanced (LTE-A), was launched in 2011 with a main goal to increase the transmission speed by 10-bold compare to $3 \mathrm{G}$ and use channel bandwidth up to 20Mhz [3]. 4G was able to achieve this goal by using multiple-input multiple-output (MIMO) technology and advanced orthogonal multiple access (OMA) techniques such as orthogonal frequency division multiplexing (OFDM). Moreover, $4 \mathrm{G}$ can achieve a data rate up to $1 \mathrm{Gbps}$ for low mobility applications and up to 100Mbps for high mobility applications [3] [4]. As mentioned before, in the coming years the traffic of wireless communication will be enormously increasing and the boundary of the $4 \mathrm{G}$ is approaching. Therefore, a lot of active researches are shifting the focus towards the fifth generation $(5 \mathrm{G})$.

The 5G systems are expected to solve problems such as poor quality of service (QoS), bad interconnectivity and poor coverage [5]. Although the 5G still a concept, its standardisation is expected to be ready by 2020. Compared to 4G, 5G systems will be designed in order to achieve a system capacity 1000 times higher and to increase the average throughput, spectral efficiency, data rate and the power efficiency by 10-bold [5]. Moreover, 5G systems should insure a system latency that is below $1 \mathrm{~ms}$ and be able to provide a data bit rate up to $10 \mathrm{Gbps}[6]$. A lot of research papers have been started to investigate the possibility of adapting the existing technologies or suggesting new ones. For example in [6], the author discusses some promising technologies for cellular architecture that can be employed for 5G systems such as femtocells, macrocells, relays and small sells. Furthermore, several recent papers have discussed some new technologies that can be deployed in $5 \mathrm{G}$ 
systems in order to achieve the expected requirements such as visible light communication (VLS), mm-wave communication, massive MIMO technology and spatial modulation (SM) technology [6] [7] [8].

According to many researches, MIMO systems are able to achieve high data rate without any increase in the transmit power or the utilization [8], [9]. However, MIMO systems will require more power amplifiers, RF chains, filters etc., which will decrease the power efficiency of the system. That is why designing a MIMO system that have a fair tradeoff between the spectral efficiency and the power efficiency is greatly needed. SM technique has proven to be one of potential technologies that can achiever this challenging task. Its basic idea is to activate one transmit antennas at each signaling interval and use the antenna index as additional modulation scheme [10]. Taking the advantage of the single-RF chain design and using the transmit antenna array, SM has shown in some recent studies that it can outperform many conventional MIMO systems with less active transmit antennas [11], $[12]$.

Another key factor that could enable future networks to achieve the best spectrum utilization is designing innovative modulation schemes. Traditionally, Orthogonal Multiple Access (OMA) techniques have been used to increase the number of users that can share the same spectrum. These OMA techniques use the orthogonal characteristic to avoid the Multiple-Access Interference (MAI), which can be reached in different domains such as frequency, time, space and code domains. Motivated by this concept, three techniques such as Direct Sequence Code Division Multiple Access (DS-CDMA), Orthogonal Frequency Domain Division (OFDM) and Time Domain Multiple Access (TDMA) have been developed and used in many standards. Based on these techniques, recent researches have proposed modified version of OMA that can be employed in future networks. These techniques include Sparse Code Multiple Access (SCMA) [13], Low Density Spreading (LDS) [14], Pattern Division Multiple Access (PDMA) [15] and Lattice Partition Multiple Access (LPMA) [16]. A recent Multiple-access technique, called Non-Orthogonal Multiple Access (NOMA), shows potential performance compared to the conventional OMA [17]. Unlike OMA where the orthogonality is the key to separate users of the same spectrum, 
NOMA encourages using non-orthogonal separation. For instance, in CDMA it is required to use different spreading codes that are orthogonal, whereas in NOMA users are allowed to use the same spreading codes. This new technique has been proposed in the $3^{\text {rd }}$ generation partnership project (3GPP) LTE standard under the name of Multiuser Superposition Transmission (MUST) [18]. Exploiting all these concepts can lead to achieve the goal and design new methods that can enhance the system performance.

\subsection{Thesis scope}

As discussed in [23], some of the main goals of the future wireless communication generations are to increase data rates and decrease system latency while keeping low power consumption. Thus, modern modulation techniques must be able to comply with these requirements. As previously discussed, SM-MIMO systems can achieve the fixed goal based on two concepts:

- Reducing the number of transmit antennas in order to decrease the power consumption by using less RF chains.

- Increasing data rates by providing additional modulation scheme.

CDMA systems were designed based on Direct-Spread Spread Spectrum (DS-SS) where each user is assigned with a unique spreading waveform called chip. This signature is used to spread the user's transmit signal over a bandwidth that is larger than its original bandwidth. At the receiver, the unique signature is used to separates users' signals. If the used spreading sequences are not orthogonal, the system will suffer from the MAI effect, which will decrease the system performance. Inspired by this concept, a modified version that uses parity bits to select the spreading waveforms is introduced in [22]. This methods shows that a coding gain can be achieved by combining coding with spreading code selection.

Combining the SM with Multiple-access techniques could improve their performance. Several works, e.g., [19], [20], studied the possibility of employing SM design with OFDM 
and SC-FDMA, but few papers has investigate the performance of SM when CDMA is used [21]. Therefore, this study focuses on designing a new SM based on parity bit CDMA technique.

\subsection{Thesis objectives}

This thesis aims to investigate the possibility of combining the SM-MIMO technique with the CDMA version introduced in [22]. As discussed in the previous sections, power and spectral efficiency are the key success of the future communication networks. SM uses the single-RF chain design to decrease the number of active transmit antennas and therefore increase the power efficiency. Moreover, it increases the data rate by using the transmit antenna array as an additional modulation scheme. In the other hand, the CDMA parity bit selection method adds a significant coding gain to the system and increases the spectral efficiency. Motivated by these two techniques, this thesis proposes an adapted version of SM-MIMO for CDMA parity bit selection system. Although, these two methods belong to two different families; however, they share the same concept of using an index variable as an additional parameter to convey the data bits. The proposed method should provide all benefits of SM-MIMO and the CDMA parity bit selection methods.

The objective of this study is to propose a modified version of the SM-MIMO technique that can be implemented using CDMA. Moreover, the thesis evaluates the bit error rate (BER) performance of the new technique under frequency-nonselective Rayleigh fading channels. The proposed system should be able to increase the BER performance compared to the conventional STBC parity bit permutation spreading design. Also, it should increase the power efficiency by proposing an optimal number of transmit antennas to activate in order to ensure a certain diversity order while reducing the number of RF-chains. Additionally, this thesis should study the MAI effect in synchronous transmission for multiuser scenarios and verify that the new design outperform the parity bit selection method of the MIMO-CDMA discussed in [22] when SM is implemented. Inspired from NOMA technique, the proposed system suggest that all users use the same spreading codes to transmit their 
data bit where quasi-orthogonal codes are used to separate the users' signals. By doing so, there will be no need to increase the length of chips used when more users are added to the same spectrum.

\subsection{Scientific methods employed}

The main purpose of this work is to provide a new design of the SM-MIMO system that can be implemented with the parity bit selection CDMA method. A mathematical model has been derived for the proposed system in order to evaluate the BER performance. The proposed system model has been tested via computer simulations under fading channels to confirm the mathematical derived model. The simulation results are compared with the theoretical results and the conventional methods in order to evaluate the overall improvement of the new method. Moreover, the system has been tested in multiuser scenarios and compared with the parity bit selection CDMA technique.

\subsection{Thesis contributions}

The key contributions of this thesis is the design of a SM-MIMO system based on the CDMA permutation spreading technique. The proposed system provides the benefits of the SM method and the permutation spreading method. It helps to increases the system performance in terms of spectral and power efficiency by reducing the number of transmit antenna to activate and using the permutation spreading method to provide additional coding gain.

The following are the key contributions of this thesis:

- Proposal of a modified version of the MIMO-CDMA spreading permutation technique when SM method is used.

- Proposal of new design strategy for the transmit antenna selection in SM-MIMO system. The design strategy is based on Space Time Block Code (STBC) permutation 
discussed in [24].

- The theoretical analysis of the BER performance of the proposed system in a single user scenario and over frequency non-selective fading channels.

- Simulations comparison and performance evaluation of the new method and the conventional methods.

- Investigation of MAI effect on the BER performance for synchronous transmission. In this scenario, all users are assumed to use the same spreading sequences and signature codes, also called pseudo-noise (PN), are used to separate the users' signals.

\subsection{Thesis organisation}

The remainder of this thesis is organised as follows:

- Chapter 2 introduces the basic idea of two parity bit selection methods for MIMOCDMA systems: parity bit selected spreading and permutation spreading techniques. In this chapter, different design strategies such as STBC design and T-design are explained. Simulations are performed to evaluate the BER performance for all the discussed techniques and compared with the theoretical expressions.

- Chapter 3 explains the concept of the SM design and how the spatial positions of transmit antennas can be used as additional modulation scheme. The chapter also discusses the generalized idea of Spatial Modulation (GNSM) where more than one transmit antennas can be activated to send same data at each signaling interval. Finally, the evaluation of the performance is done through simulation results for different scenarios.

- Chapter 4 introduces the proposed technique and shows how the SM design could be combined with permutation spreading method in order to increase the system performance. Moreover, an analytical expression of the BER is derived for the proposed technique under frequency non-selective Rayleigh fading channels with independent 
gains and perfect knowledge of the channel state information (CSI) at the receiver. Finally, a comparison between the proposed technique and the conventional STBC and SM design is presented and discussed.

- Chapter 5 discussed the multiusers scenario of the proposed system. In this chapter, the effect of MAI are discussed when all users use the same spreading sequences and scrambling codes are used to separate users from each other. Finally, simulation results are presented and discussed.

- Chapter 6 provides a brief summary of the thesis and addresses some potential subjects for future research. 


\section{Chapter 2}

\section{Permutation Spreading For MIMO-CDMA System}

\section{$2.1 \quad$ Introduction}

The MIMO-CDMA permutation spreading technique [22] is an extension of the parity bit selected spreading technique that was first introduced by C. D'Amours in [24]. In the later method [24], instead of appending the parity bits of a systematic $(n, k)$ linear block code to the end of the information sequence, they are used to choose a spreading waveform from a set of $2^{n-k}$ mutually orthogonal sequences assigned to the user. Simulation results show better BER performance of this technique compared to the conventional Direct Sequence Spread Spectrum (DS-SS) methods [24].

The application of the parity bit selected spreading technique to the MIMO-CDMA systems is introduced in [22] where two new methods are proposed. For the conventional MIMO-CDMA system the spreading sequences are fixed regardless the data to be transmitted. The authors then propose two methods where the spreading codes dependent on the data being transmitted. The first proposed method is MIMO-CDMA employing parity bit selected spreading, the parity bits are used to select the spreading sequence to be assigned during one signaling interval to all transmit antennas from a set of mutually orthogonal waveforms. In the second method proposed in [22] is the permutation spreading where the 
parity bits are used to select one of $N_{t}$ different spreading sequences from a set of mutually orthogonal spreading sequences; and each antenna uses one of the selected sequences during one signaling interval.

Many methods exist for designing the spreading permutation sequences. In [22] a Tdesign is used where the rule of designing the spreading sequences is that if an antenna $\mathrm{j}$ uses a spreading sequence in one permutation, it cannot use the same spreading sequence in any other permutations. The spreading sequence can be reused in a different permutation but by a different transmit antenna. In [25] another design technique based on space-time block code (STBC) matrices is proposed. This method shows a slight BER performance improvement compared to T-design.

\subsection{System Model}

\subsubsection{Transmitter Model}

The transmitter block diagram of a MIMO-CDMA system using parity bit selected and permutation spreading is shown in Figure 2.1. In this model, the information bits are first segmented into message blocks of $k$ bits, where $k=\log _{2}(M) * N_{t}$ for M-ary signal mapping. The message blocks are then converted into $N_{t}$ parallel streams. During one signaling interval, the message bits are modulated using binary phase shift keying (BPSK) and at the same time fed into the spreading sequence selector. Based on the input data, the spreading sequences to be used on each transmit antenna $\left(w_{1}(t), w_{2}(t), \ldots, w_{N_{t}}(t)\right)$, are selected from $N$ set of mutually orthogonal sequences $\left(c_{1}(t), c_{2}(t), \ldots, c_{N}(t)\right)$, where $N \geqslant N_{t}$. The chosen spreading sequences will be then multiplied with the message symbols $\left(s_{1}, s_{2}, \ldots, s_{N_{t}}\right)$ before transmission.

The basic idea of the design strategy is to divide the message vector into different cosets $\left(M_{1}, M_{2}, \ldots, M_{K}\right)$ that will provide a maximum Euclidian distance between messages in each coset. The number of cosets $(K)$ depends on the number of antennas $\left(N_{t}\right)$ and the number of messages in each coset $(L): K=2^{N_{t}} / L$. 


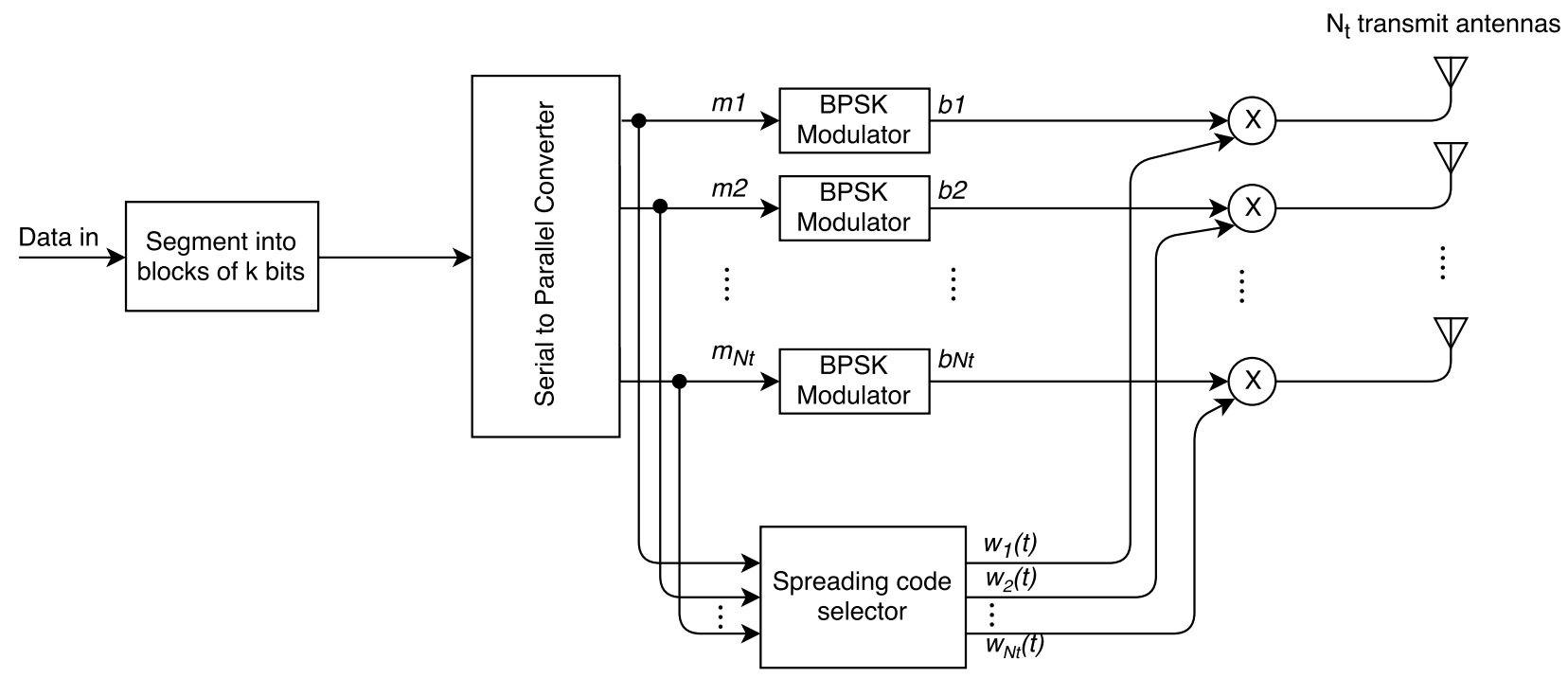

Figure 2.1: Transmitter of MIMO-CDMA with parity bit selected and permutation spreading

If the parity bit selected spreading method is used, each coset will be assigned one spreading waveform that will be used by all transmit antennas. In the system presented in [22], 4 transmit antennas are used with 2 messages per coset. In this case, 8 cosets are generated: $M_{1}=\{0000 ; 1111\}, M_{2}=\{0001 ; 1110\}, M_{3}=\{0010 ; 1101\}, M_{4}=\{0011 ; 1100\}$, $M_{5}=\{0100 ; 1011\}, M_{6}=\{0101 ; 1010\}, M_{7}=\{0110 ; 1001\}$ and $M_{8}=\{0111 ; 1000\}$. For instance, if a message vector to transmit is $\left[\begin{array}{llll}0 & 1 & 1 & 0\end{array}\right]$, all transmit antennas will use the spreading waveform associated with coset $M_{7}$. Thus, 8 mutually orthogonal waveforms are needed.

In the case of MIMO-CDMA systems using permutation spreading, each coset has a unique permutation spreading waveforms. Each permutation uses $N_{t}$ of the $N$ mutually orthogonal spreading sequences in such a way that all permutations have the minimum possible waveforms in common. Moreover, a transmit antenna $j$ can use the same waveform only once in all permutations. The first permutation design was introduced in [22] and is based on T-design [26]. Tables 2.1 shows the spreading permutations where $w_{j}(t)$ refers to the spreading waveform assigned to antenna $\mathrm{j}$ and $c_{i}(t)$ is the $i t h$ spreading code. In this system, 4 transmit antennas are used with 2 messages per coset. Hence, 8 orthogonal wave- 
forms are available. Each permutation of a specific coset shares one spreading waveform with two other permutations and two spreading waveforms with five other permutations.

The main advantage of this design is that it creates dependence between parallel data stream by the use of different spreading patterns and without losing orthogonality between streams.

\begin{tabular}{|c|c|c|c|c|c|}
\hline Coset & Message Vector & $\boldsymbol{w}_{\mathbf{1}}(\boldsymbol{t})$ & $\boldsymbol{w}_{\mathbf{2}}(\boldsymbol{t})$ & $\boldsymbol{w}_{\mathbf{3}}(\boldsymbol{t})$ & $\boldsymbol{w}_{\mathbf{4}}(\boldsymbol{t})$ \\
\hline$M_{1}$ & 0000 & $c_{1}(t)$ & $c_{3}(t)$ & $c_{5}(t)$ & $c_{7}(t)$ \\
\hline$M_{2}$ & 1111 & $c_{8}(t)$ & $c_{1}(t)$ & $c_{4}(t)$ & $c_{5}(t)$ \\
\hline$M_{3}$ & 1110 & $c_{2}(t)$ & $c_{4}(t)$ & $c_{3}(t)$ & $c_{8}(t)$ \\
\hline$M_{4}$ & 0001 & $c_{5}(t)$ & $c_{2}(t)$ & $c_{6}(t)$ & $c_{3}(t)$ \\
\hline$M_{5}$ & 0011 & $c_{6}(t)$ & $c_{7}(t)$ & $c_{1}(t)$ & $c_{4}(t)$ \\
\hline$M_{6}$ & 1100 & $c_{3}(t)$ & $c_{6}(t)$ & $c_{8}(t)$ & $c_{1}(t)$ \\
\hline$M_{7}$ & 10100 & $c_{7}(t)$ & $c_{8}(t)$ & $c_{2}(t)$ & $c_{6}(t)$ \\
\hline$M_{8}$ & 1010 & $c_{4}(t)$ & $c_{5}(t)$ & $c_{7}(t)$ & $c_{2}(t)$ \\
\hline
\end{tabular}

Table 2.1: T-Design Permutations Spreading for MIMO-CDMA system with 4 transmit antennas

Another permutation design is discussed in [25] which is based on the Alamouti's orthogonal space-time block code (STBC) matrix. [25] shows that this strategy, in addition to keeping the orthogonality between message vectors, can achieve the same diversity order as STBC. For a system with 4 transmit antenna 8 orthogonal waveforms are needed, and thus the permutation table is generated based on the full rate ${ }^{1} 8 \mathrm{x} 8 \mathrm{STBC}$ matrix 2.1 given

\footnotetext{
${ }^{1}$ Code rate is equal to 1.
} 
in $[27]$.

$$
\left(\begin{array}{rrrrrrrr}
s_{1} & s_{2} & s_{3} & s_{4} & s_{5} & s_{6} & s_{7} & s_{8} \\
-s_{2} & s_{1} & s_{4} & -s_{3} & s_{6} & -s_{5} & -s_{8} & s_{7} \\
-s_{3} & s_{4} & s_{1} & s_{2} & s_{7} & s_{8} & -s_{5} & -s_{6} \\
-s_{4} & s_{3} & s_{2} & s_{1} & s_{8} & -s_{7} & s_{6} & -s_{5} \\
-s_{5} & s_{6} & s_{7} & -s_{8} & s_{1} & s_{2} & s_{3} & s_{4} \\
-s_{6} & s_{5} & s_{8} & s_{7} & -s_{2} & s_{1} & -s_{4} & s_{3} \\
-s_{7} & s_{8} & s_{5} & -s_{6} & -s_{3} & s_{4} & s_{1} & -s_{2} \\
-s_{8} & s_{7} & s_{6} & s_{5} & -s_{4} & -s_{3} & s_{2} & s_{1}
\end{array}\right)
$$

The spreading permutation table for 4 transmit antennas is given as follows ${ }^{2}$ :

\begin{tabular}{|c|c|c|c|c|c|}
\hline Coset & Message Vector & $\boldsymbol{w}_{\mathbf{1}}(\boldsymbol{t})$ & $\boldsymbol{w}_{\mathbf{2}}(\boldsymbol{t})$ & $\boldsymbol{w}_{\mathbf{3}}(\boldsymbol{t})$ & $\boldsymbol{w}_{\mathbf{4}}(\boldsymbol{t})$ \\
\hline$M_{1}$ & 0000 & $c_{1}(t)$ & $c_{5}(t)$ & $c_{8}(t)$ & $c_{6}(t)$ \\
\hline$M_{2}$ & 0001 & $c_{2}(t)$ & $c_{6}(t)$ & $c_{7}(t)$ & $c_{5}(t)$ \\
\hline$M_{3}$ & 00110 & $c_{3}(t)$ & $c_{7}(t)$ & $c_{6}(t)$ & $c_{8}(t)$ \\
\hline$M_{4}$ & 1101 & $c_{4}(t)$ & $c_{8}(t)$ & $c_{5}(t)$ & $c_{7}(t)$ \\
\hline$M_{5}$ & 0011 & $c_{5}(t)$ & $c_{1}(t)$ & $c_{4}(t)$ & $c_{2}(t)$ \\
\hline$M_{6}$ & 1100 & $c_{6}(t)$ & $c_{2}(t)$ & $c_{3}(t)$ & $c_{1}(t)$ \\
\hline$M_{7}$ & 0100 & $c_{7}(t)$ & $c_{3}(t)$ & $c_{2}(t)$ & $c_{4}(t)$ \\
\hline$M_{8}$ & 1011 & $c_{8}(t)$ & $c_{4}(t)$ & $c_{1}(t)$ & $c_{3}(t)$ \\
\hline
\end{tabular}

Table 2.2: STBC-Design Permutations Spreading for MIMO-CDMA system with 4 transmit antennas

A more specific transmit signal table can be generated using the above concept. For

${ }^{2}$ Only 4 column from the $8 \times 8$ STBC matrix are needed to generate the permutations table. In [25] column 1, 5, 8 and 6 are used. Other combination may be used as all columns in the STBC matrix are orthogonal to each other. 
example let us consider a MIMO-CDMA system with 4 transmit antennas using BPSK modulation. If the message vector to be transmitted is $m=\left[\begin{array}{llll}0 & 0 & 0 & 0\end{array}\right]$ from coset $M_{1}$, then the transmit signal from antenna 1, 2,3 and 4 are given by:

$$
\begin{aligned}
& m_{1} * w_{1}(t)=-c_{1}(t) \\
& m_{2} * w_{2}(t)=-c_{5}(t) \\
& m_{3} * w_{3}(t)=-c_{8}(t) \\
& m_{4} * w_{4}(t)=-c_{6}(t)
\end{aligned}
$$

where $m_{j}$ is the bit transmitted from the $j t h$ transmit antenna; and the $w_{j}(t)$ is the spreading waveform assigned to antenna $j$.

Now, if the message vector to be transmitted is $m=\left[\begin{array}{llll}1 & 1 & 1 & 1\end{array}\right]$ from the same coset $M_{1}$, then the transmit signal from antenna 1,2, 3 and 4 are given by:

$$
\begin{aligned}
& m_{1} * w_{1}(t)=c_{1}(t) \\
& m_{2} * w_{2}(t)=c_{5}(t) \\
& m_{3} * w_{3}(t)=c_{8}(t) \\
& m_{4} * w_{4}(t)=c_{6}(t)
\end{aligned}
$$

The full transmit signals table is given as follows:

\subsubsection{Receiver Model}

Figure 2.2 shows the receiver block diagram of a MIMO-CDMA system using parity bit selected and permutation spreading. Each receiver antenna is connected to a bank of matched filters that corresponds to one of the $N$ spreading waveform $\left\{c_{1}(t), c_{2}(t) \ldots, c_{N}(t)\right\}$. The estimated transmitted bits are determined using the outputs of the matched filters as decision variables and Maximum Likelihood (ML) as detection method [22]. Although the receiver model looks to be similar for both techniques, the decision device is different.

\section{Decision variable for MISO-CDMA system with Permutation Spreading}

To determine the decision variable let us first consider a MISO-CDMA system with parity bit selected spreading sequences using BPSK modulation with $N_{t}$ transmit antennas 


\begin{tabular}{|c|c|c|c|c|c|}
\hline Coset & Message Vector & $\boldsymbol{w}_{\mathbf{1}}(\boldsymbol{t})$ & $\boldsymbol{w}_{\mathbf{2}}(\boldsymbol{t})$ & $\boldsymbol{w}_{\mathbf{3}}(\boldsymbol{t})$ & $\boldsymbol{w}_{\mathbf{4}}(\boldsymbol{t})$ \\
\hline \multirow{3}{*}{$M_{1}$} & 0000 & $c_{1}(t)$ & $c_{5}(t)$ & $c_{8}(t)$ & $c_{6}(t)$ \\
\cline { 2 - 6 } & 1111 & $-c_{1}(t)$ & $-c_{5}(t)$ & $-c_{8}(t)$ & $-c_{6}(t)$ \\
\hline \multirow{3}{*}{$M_{2}$} & 0001 & $-c_{2}(t)$ & $-c_{6}(t)$ & $-c_{7}(t)$ & $c_{5}(t)$ \\
\cline { 2 - 6 } & 1110 & $c_{2}(t)$ & $c_{6}(t)$ & $c_{7}(t)$ & $-c_{5}(t)$ \\
\hline \multirow{3}{*}{$M_{3}$} & 0010 & $-c_{3}(t)$ & $-c_{7}(t)$ & $c_{6}(t)$ & $-c_{8}(t)$ \\
\cline { 2 - 6 } & 1101 & $c_{3}(t)$ & $c_{7}(t)$ & $-c_{6}(t)$ & $c_{8}(t)$ \\
\hline \multirow{3}{*}{$M_{4}$} & 0011 & $-c_{4}(t)$ & $-c_{8}(t)$ & $c_{5}(t)$ & $c_{7}(t)$ \\
\cline { 2 - 6 } & 1100 & $c_{4}(t)$ & $c_{8}(t)$ & $-c_{5}(t)$ & $-c_{7}(t)$ \\
\hline \multirow{3}{*}{$M_{5}$} & 0100 & $-c_{5}(t)$ & $c_{1}(t)$ & $-c_{4}(t)$ & $-c_{2}(t)$ \\
\cline { 2 - 6 } & 1011 & $c_{5}(t)$ & $-c_{1}(t)$ & $c_{4}(t)$ & $c_{2}(t)$ \\
\hline \multirow{3}{*}{$M_{6}$} & 0101 & $-c_{6}(t)$ & $c_{2}(t)$ & $-c_{3}(t)$ & $c_{1}(t)$ \\
\cline { 2 - 6 } & 1010 & $c_{6}(t)$ & $-c_{2}(t)$ & $c_{3}(t)$ & $-c_{1}(t)$ \\
\hline \multirow{2}{*}{$M_{7}$} & 0110 & $-c_{7}(t)$ & $c_{3}(t)$ & $c_{2}(t)$ & $-c_{4}(t)$ \\
\cline { 2 - 6 } & 1001 & $c_{7}(t)$ & $-c_{3}(t)$ & $-c_{2}(t)$ & $c_{4}(t)$ \\
\hline \multirow{2}{*}{$M_{8}$} & 0111 & $-c_{8}(t)$ & $c_{4}(t)$ & $c_{1}(t)$ & $c_{3}(t)$ \\
\cline { 2 - 6 } & 1000 & $c_{8}(t)$ & $-c_{4}(t)$ & $-c_{1}(t)$ & $-c_{3}(t)$ \\
\hline
\end{tabular}

Table 2.3: STBC-Design Permutations Spreading transmit table for 4 transmit antennas

and 1 receive antenna. The received signal from a message vector $(k)$, at each signalling interval, is the sum of all bits multiplied by corresponding waveform and the channels gain and it can be expressed as:

$$
r_{x_{\text {parity bit }}^{(k)}}^{\left(N_{t}\right.}=\sum_{i=1}^{N_{E}} \sqrt{E_{b}} b_{i}^{(k)} \alpha_{1 i}^{(k)} w^{(k)}+n^{(k)}
$$

with $E_{b}$ is the received energy per bit; $\alpha_{1 i}^{(k)}$ is the complex channel gain between the $i t h$ transmit antenna and the received antenna; $b_{i}^{(k)}$ is the message bits transmitted over the $i t h$ transmit antenna from the $k t h$ message vector; $w^{(k)}$ is the spreading sequence corresponding to message vector $k ; n^{(k)}$ is the receiver noise when receiving the $k t h$ message.

The output of the lth matched filter at the receive antenna during one signaling interval is: 


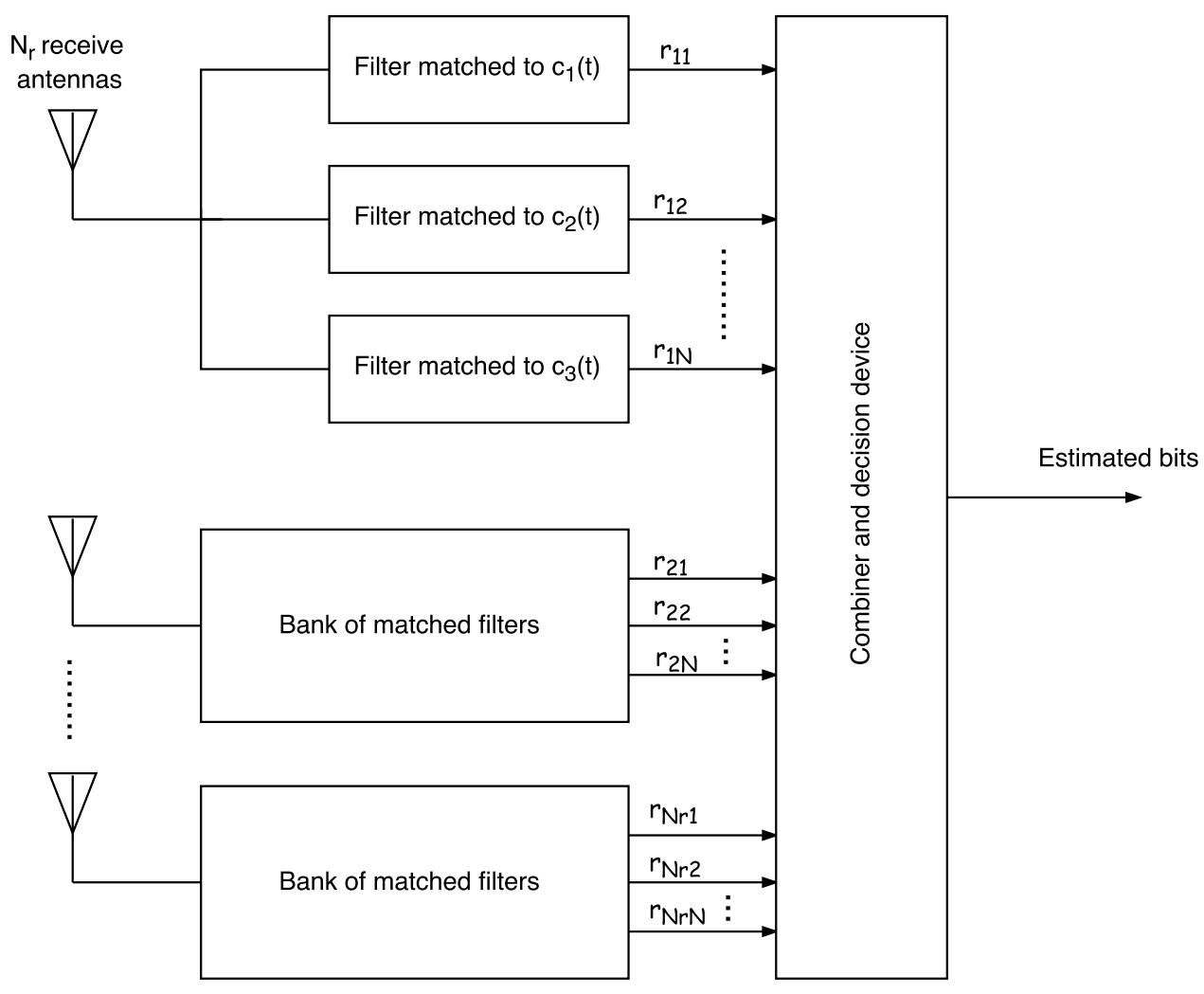

Figure 2.2: Receiver of MIMO-CDMA with parity bit selected and permutation spreading

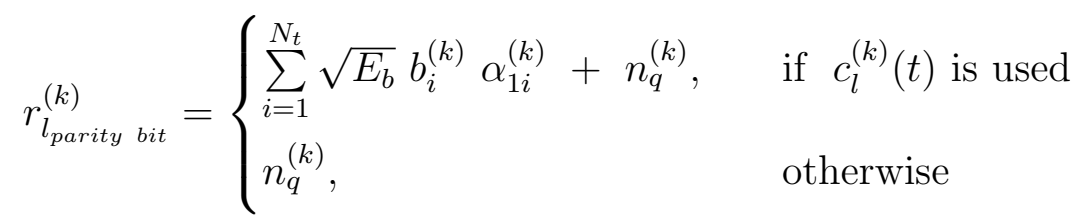

where $t \in[0, T], T$ is the bit duration and $n_{l}$ is the sampled noise after the lth matched filter, which is expressed as:

$$
n_{l}^{(k)}=\frac{1}{T} \int_{T} n_{k}^{(k)} c_{l}^{k}(t) d t
$$

ML detection is used in order to estimate data bits when the $k$ th message vector is transmitted; which is done by finding the spreading sequence that provides the minimum Euclidian distance between the received message vector and all possible message vectors. 


$$
U=\min _{j=1}^{M}\left\|r_{x_{\text {parity } b i t}^{(k)}}^{\left(N_{t}\right.}-\sum_{i=1}^{E_{b}} b_{i}^{(k)} \alpha_{1 i}^{(k)} c_{i}^{(k)}\right\|^{2}
$$

where $\mathrm{M}$ is the total number of cosets.

Similarly, for MISO-CDMA system with permutation spreading the received message vector can be expressed as:

$$
r_{x_{p e r m}}^{(k)}=\sum_{i=1}^{N_{t}} \sqrt{E_{b}} b_{i}^{(k)} \alpha_{1 i} w_{i}^{(k)}+n^{(k)}
$$

with $w_{i}^{(k)}$ is the spreading sequence assigned to the $i t h$ antenna when the $k t h$ message vector is transmitted. The output of the $l t h$ matched filter at the receive antenna during one time instant is:

$$
r_{l_{\text {perm }}}^{(k)}= \begin{cases}\sum_{i=1}^{N_{t}} \sqrt{E_{b}} b_{i}^{(k)} \alpha_{1 i}^{(k)}+n_{q}^{(k)}, & \text { if } w_{i}^{(k)}(t)=c_{i}^{(k)}(t) \\ n_{q}^{(k)}, & \text { otherwise }\end{cases}
$$

In this case, the decision variable is expressed as follows:

$$
U=\min _{j=1}^{M}\left\|r_{x_{p e r m}}^{(k)}-\sum_{i=1}^{N_{t}} \sqrt{E_{b}} b_{i}^{(k)} \alpha_{1 i}^{(k)} w_{i}^{(k)}\right\|^{2}
$$

\section{Decision variable for MIMO-CDMA system with Permutation Spreading}

We notice that the decision variable is similar for both parity bit selected and permutations spreading, except in the spreading sequence selection phase. For this reason, only MIMO-CDMA system with permutation spreading sequences using BPSK modulation with $N_{t}$ transmit antennas and $N_{r}$ receive antennas is considered in this section. The 
output of the $l t h$ matched filter at the $j t h$ receive antenna during one signalling interval is:

$$
r_{j l}^{(k)}= \begin{cases}\sum_{i=1}^{N_{t}} \sqrt{\frac{E_{b}}{N_{r}}} b_{i}^{(k)} \alpha_{i j}^{(k)}+n_{j q}^{(k)}, & \text { if } w_{i}^{(k)}(t)=c_{i}^{(k)}(t) \\ n_{j q}^{(k)}, & \text { otherwise }\end{cases}
$$

with $E_{b}$ is the received energy per bit; $\alpha_{i j}$ is the complex channel gain between the $i t h$ transmit antenna and the $j$ th received antenna; $b_{i}^{(k)}$ is the $i t h$ message bits in the $k t h$ message vector; $w_{i}$ is the spreading sequence corresponding to message vector $k ; n_{j l}^{(k)}$ is the sampled noise after the $l t h$ matched at the $j t h$ receive antenna.

The received vector is expressed as follows:

$$
\boldsymbol{r}_{\boldsymbol{x}}=\left[r_{11}, r_{12}, \ldots, r_{1 N}, r_{21}, r_{22}, \ldots, r_{2 N}, \ldots, r_{N_{r} 1}, r_{N_{r} 2}, \ldots, r_{N_{r} N}\right]^{T}=\boldsymbol{u}_{\boldsymbol{b}}+\boldsymbol{n}
$$

where $\boldsymbol{u}_{\boldsymbol{b}}$ is the $1 \mathrm{x} N . N_{r}$ received data matrix that depends on the transmitted data vector $\boldsymbol{b}=\left[b 1, b 2, \ldots, b_{N_{t}}\right]$. For instance if the message to be transmitted, using the STBCdesign based on Table 2.3, is $\boldsymbol{m}=\left[\begin{array}{llll}1 & 1 & 1 & 0\end{array}\right]$, then $b=\left[\begin{array}{llll}1 & 1 & 1 & -1\end{array}\right]$ and

$$
\boldsymbol{u}_{\boldsymbol{b}}=\left[0, \alpha_{11}, 0,0,-\alpha_{14}, \alpha_{12}, \alpha_{13}, 0, \ldots, 0, \alpha_{N_{r} 1}, 0,0,-\alpha_{N_{r} 4}, \alpha_{N-r 2}, \alpha_{N-r 3}, 0\right]^{T}
$$

$\boldsymbol{n}$ is the $1 \mathrm{x} N . N_{r}$ noise matrix $\boldsymbol{n}=\left[n_{11}, \ldots, n_{1 N}, n_{21}, \ldots, n_{2 N}, \ldots, n_{N r 1}, . ., n_{N_{r} N}\right]^{T}$.

ML detection is used to estimate the data bits when the $k t h$ message vector is transmitted. The expression of the decision variable is as follows:

$$
U=\min _{j=1}\left\|\boldsymbol{r}_{\boldsymbol{x}}-\boldsymbol{u}_{\boldsymbol{b}}\right\|^{2}
$$

where $\boldsymbol{u}_{\boldsymbol{b}}$ is the vector made up of all possible received vector with the absence of the noise. 


\subsection{Theoretical analysis of the BER performance}

\subsubsection{BER analysis for MIMO-CDMA system with parity bit selected spreading}

In [28] an analytical expression is shown for the union bound of the BER for a MIMOCDMA system employing parity bit selected spreading. The BER is determined by integrating the probability density function (pdf) of the energy per bit to noise power density ratio $\left(E_{b} / N_{0}\right)$ multiplied by a Q-function. The pdf of $E_{b} / N_{0}$ is shown, in [28], to be a chi-square distribution with $2 L$ degree of freedom for a MIMO system with $N_{t}$ transmit antenna and $N_{r}$ receive antenna. The integration result has the form of the following expression [29]:

$$
p(L, x)=\left(\frac{1-\mu}{2}\right)^{L} \quad \sum_{k=0}^{L-1}\left(\begin{array}{c}
L-1+k \\
k
\end{array}\right)\left(\frac{1+\mu}{2}\right)^{k}
$$

where

$$
\mu=\sqrt{\frac{x}{x+1}}
$$

Study in [28] shows that the BER of a MIMO system employing parity bit selected spreading sequence can be determined by, first, finding the squared Euclidian distance, between constellation points, then computing the union bound of the BER. This holds true under the following assumptions:

- Channels are assumed to be frequency-nonselective.

- Channel gains are independent, slowly varying and are known at the receiver.

- Maximum likelihood detection is used.

- Four transmit and received antennas and eight spreading waveforms are used. 
Under these conditions, the union bound of the probability of bit for MIMO-CDMA system employing parity bit selected spreading sequence is given by:

$$
P_{b}=p_{1}+4 p_{2}+3 p_{3}
$$

with

$$
\begin{gathered}
p_{1}=p\left(N_{r}, \frac{4}{N_{r}} \frac{E_{b}}{N_{0}}\right) \\
p_{2}=p\left(2 N_{r}, \frac{1}{N_{r}} \frac{E_{b}}{N_{0}}\right) \\
p_{3}=\frac{81}{16} p\left(4, \frac{3}{8} \frac{E_{b}}{N_{0}}\right)+\frac{1}{16} p\left(4, \frac{1}{8} \frac{E_{b}}{N_{0}}\right)-\frac{81}{8} p\left(3, \frac{3}{8} \frac{E_{b}}{N_{0}}\right)+\frac{3}{8} p\left(3, \frac{1}{8} \frac{E_{b}}{N_{o}}\right) \\
+\frac{405}{32} p\left(2, \frac{3}{8} \frac{E_{b}}{N_{o}}\right)+\frac{405}{32} p\left(1, \frac{3}{8} \frac{E_{b}}{N_{o}}\right)+\frac{45}{32} p\left(2, \frac{1}{8} \frac{E_{b}}{N_{o}}\right)-\frac{135}{32} p\left(1, \frac{1}{8} \frac{E_{b}}{N_{o}}\right)
\end{gathered}
$$

\subsubsection{BER analysis for MIMO-CDMA system with Permutation Spreading}

Same analysis of [28] is done in [22] to determine the union bound of the probability of bit for a CDMA-MIMO system employing permutation spreading sequences based on STBC design. The channels are assumed to be frequency non-selective with independent gains. The bit error probability is determined by, first, finding the squared Euclidian distance, between message vectors, then computing the union bound on the BER:

$$
P_{b}=p_{\text {same }}+\left(\frac{M-2}{2}\right) p_{\text {diff }}
$$

where $M$ is the number of message vectors and $p_{\text {same }}$ is the probability of error when the receiver detects the correct spreading sequence but not the same data vector. $p_{\text {same }}$ is given as follows:

$$
p_{\text {same }}=p\left(N_{t} N_{r}, \frac{1}{N_{r}} \frac{E_{b}}{N_{0}}\right)
$$


$p_{\text {diff }}$ is the probability of error when the receiver detects the incorrect spreading sequence. $p_{\text {diff }}$ is given as follows:

$$
p_{\text {diff }}=p\left(N_{t} N_{r}, \frac{1}{2 N_{r}} \frac{E_{b}}{N_{0}}\right)
$$

with $p(L, x)$ is computed using equation 2.14 .

\subsection{Simulation results and discussion}

Performance of the bit error rate (BER) of the three techniques discussed in this chapter is presented in this section. All simulations are performed using Matlab where the following assumption are considered:

- Orthogonal spreading codes are generated using Hadamard codes with length of 8 bits per chip sequence.

- All channel gains are considered to be independent, frequency non-selective and slowly varying complex Gaussian random variables with zero means and unit variance.

- Perfect channel state information (CSI) is considered to be available at the receiver with zero power penalty.

- BPSK modulation is used for all discussed methods and data bits are generated such a way to ensure at least 100 errors counted.

The simulations are performed considering two scenarios. In the first one a MISOCDMA system with two and four transmit antennas is considered. In the second scenario, a MIMO-CDMA system with two and four transmit antennas and two and four received antenna is considered. Simulation results are shown in Figures 2.3 and 2.4 for Parity Bit Selected spreading and in Figures 2.5 and 2.6 for permutation spreading method. A comparison of the BER performance between the three techniques is shown in Figures 2.7 and 2.8 . 


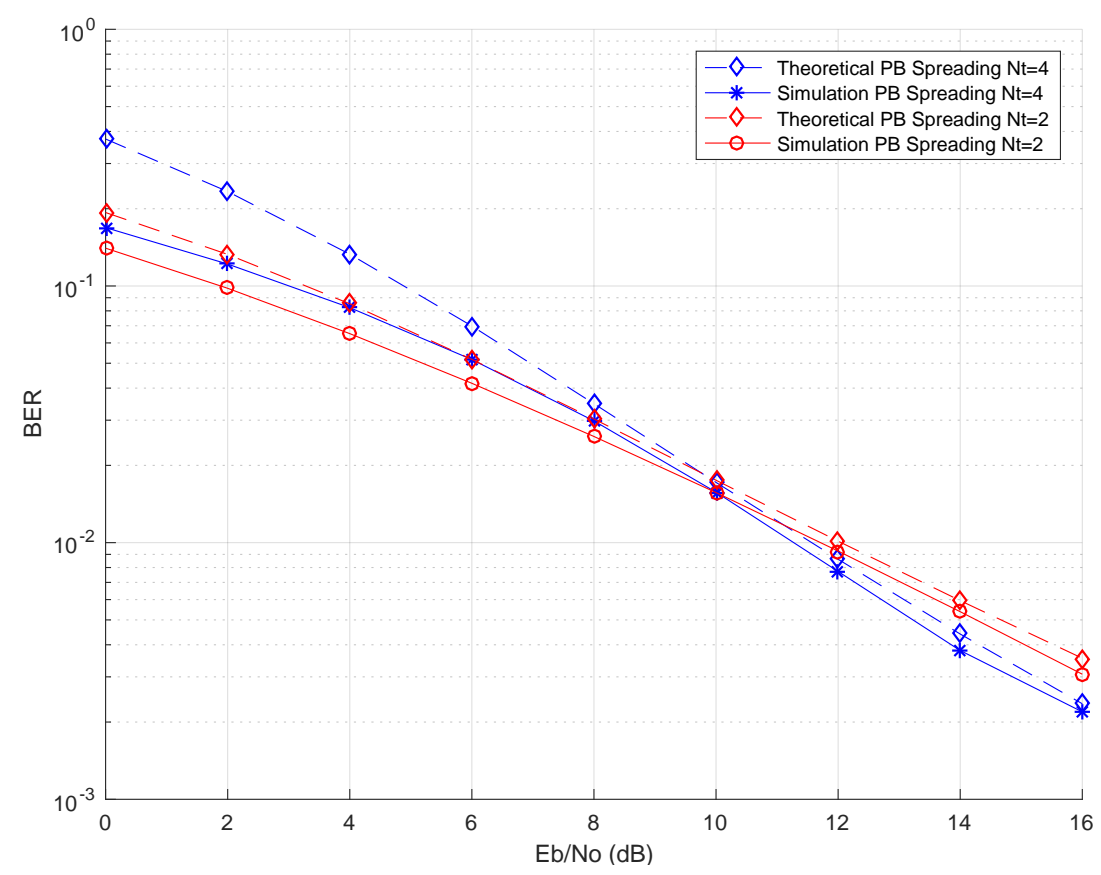

Figure 2.3: BER performance of MISO-CDMA System with parity bit selected spreading

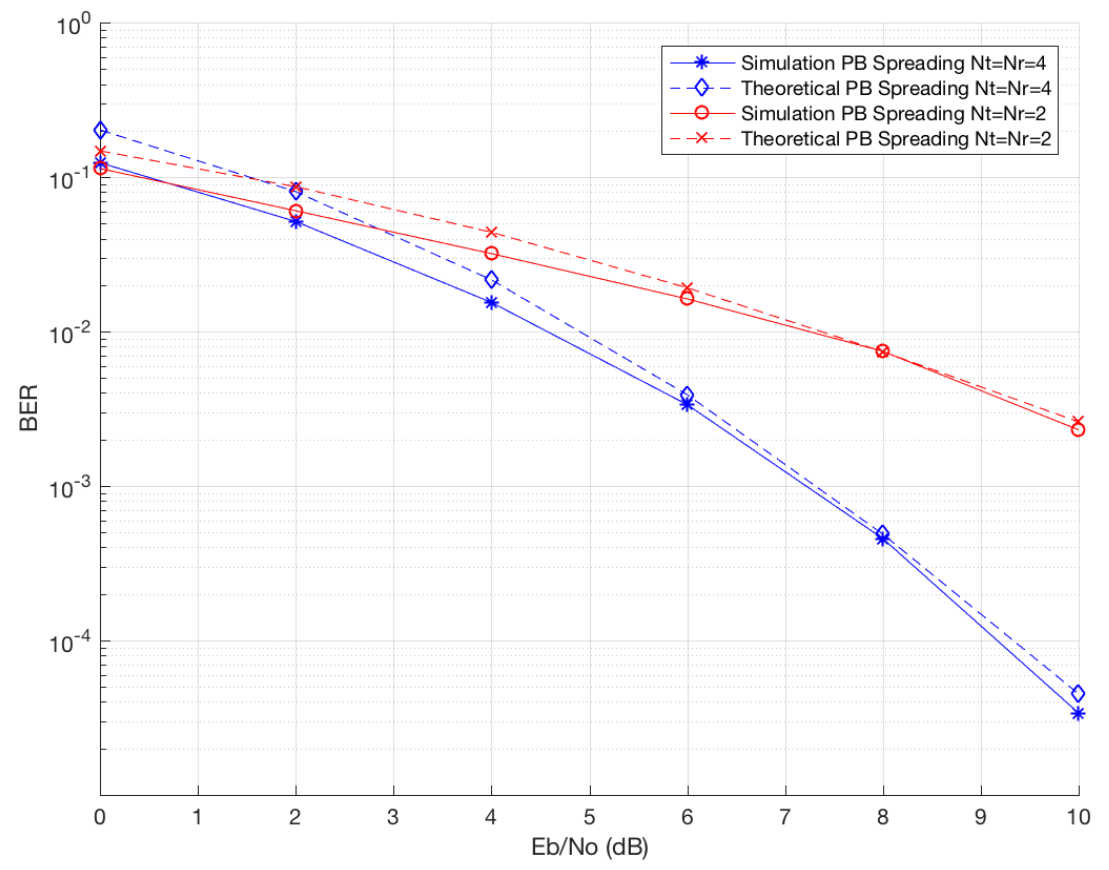

Figure 2.4: BER performance of MIMO-CDMA System with parity bit selected spreading 


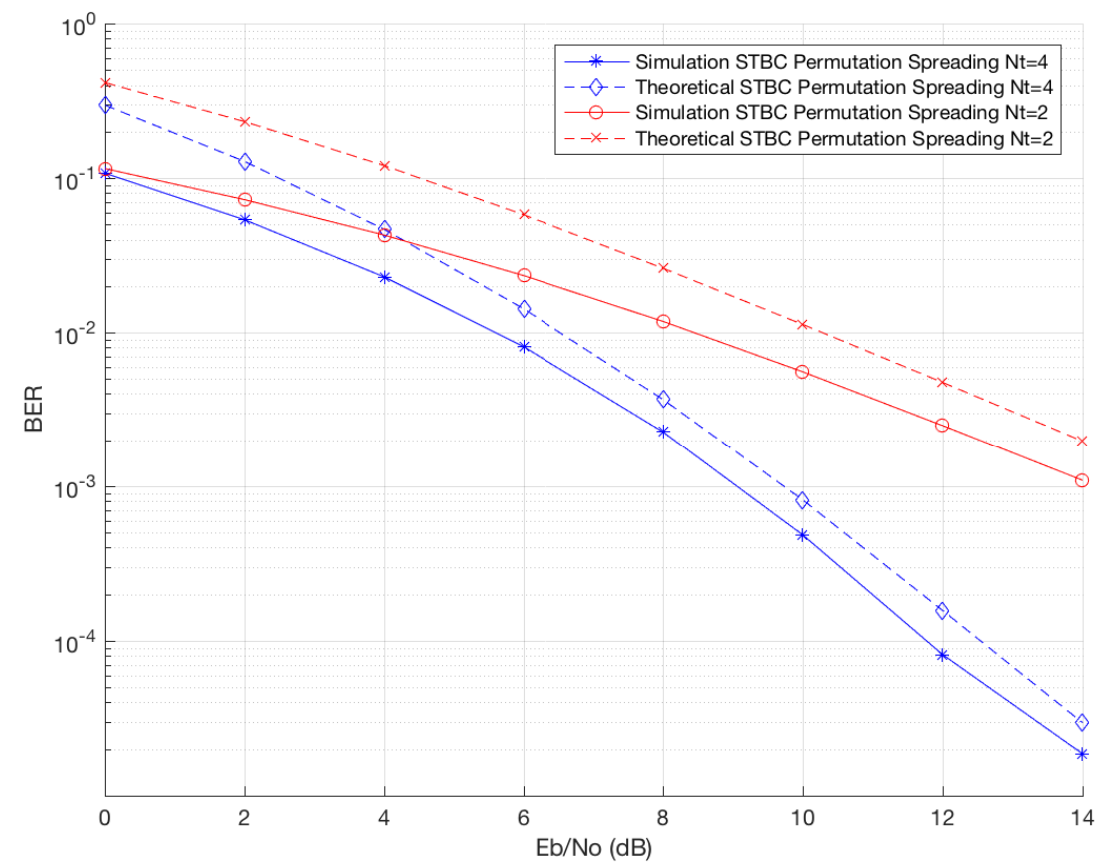

Figure 2.5: BER performance of MISO-CDMA System with STBC permutation spreading

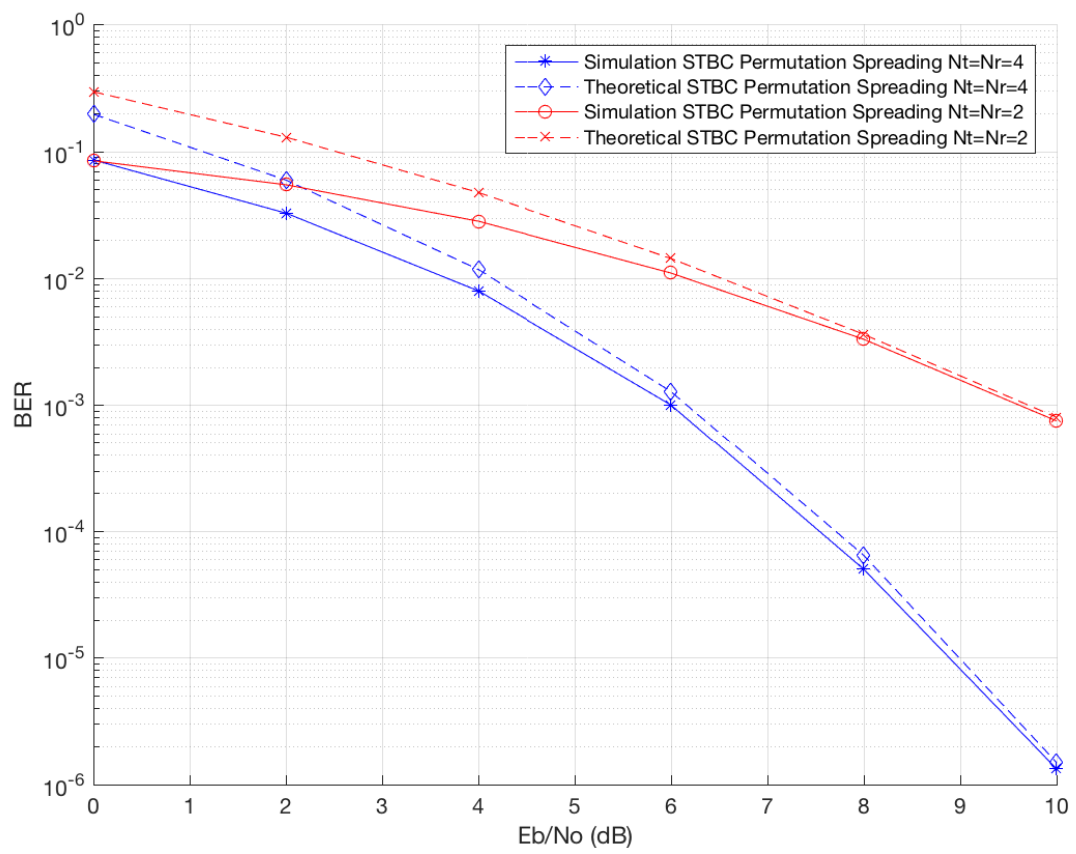

Figure 2.6: BER performance of MIMO-CDMA System with STBC permutation spreading 


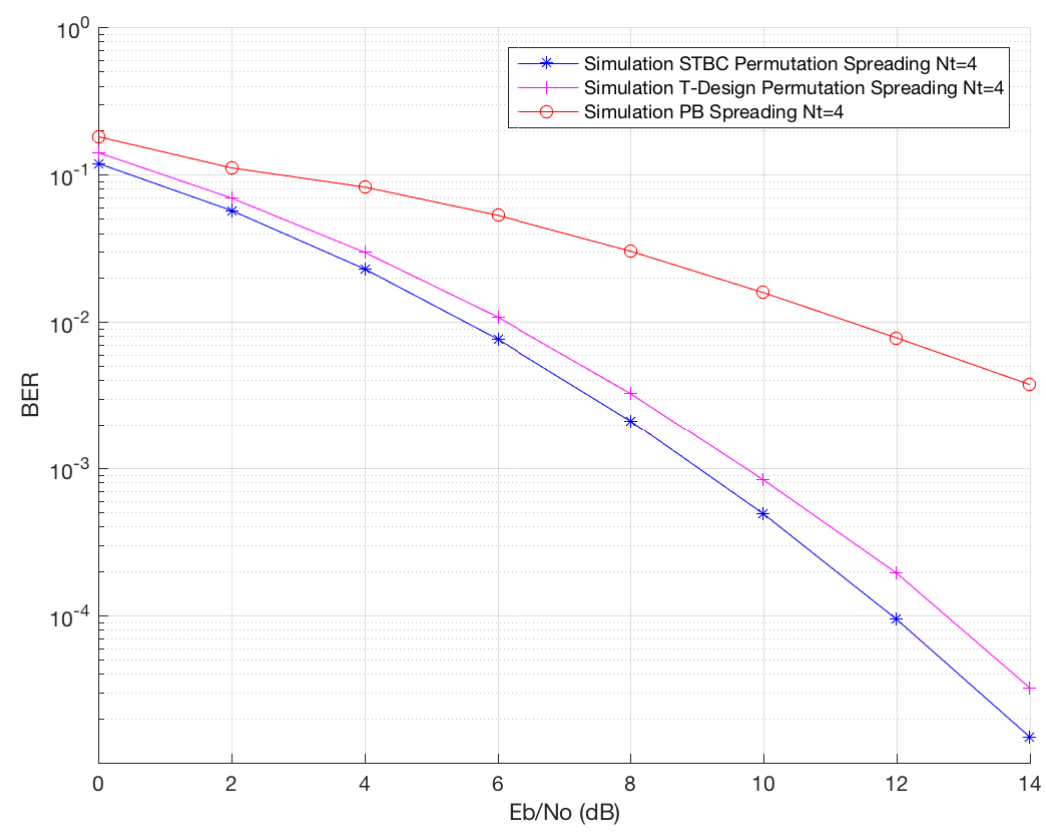

Figure 2.7: BER performance comparison of MISO-CDMA System using the three techniques

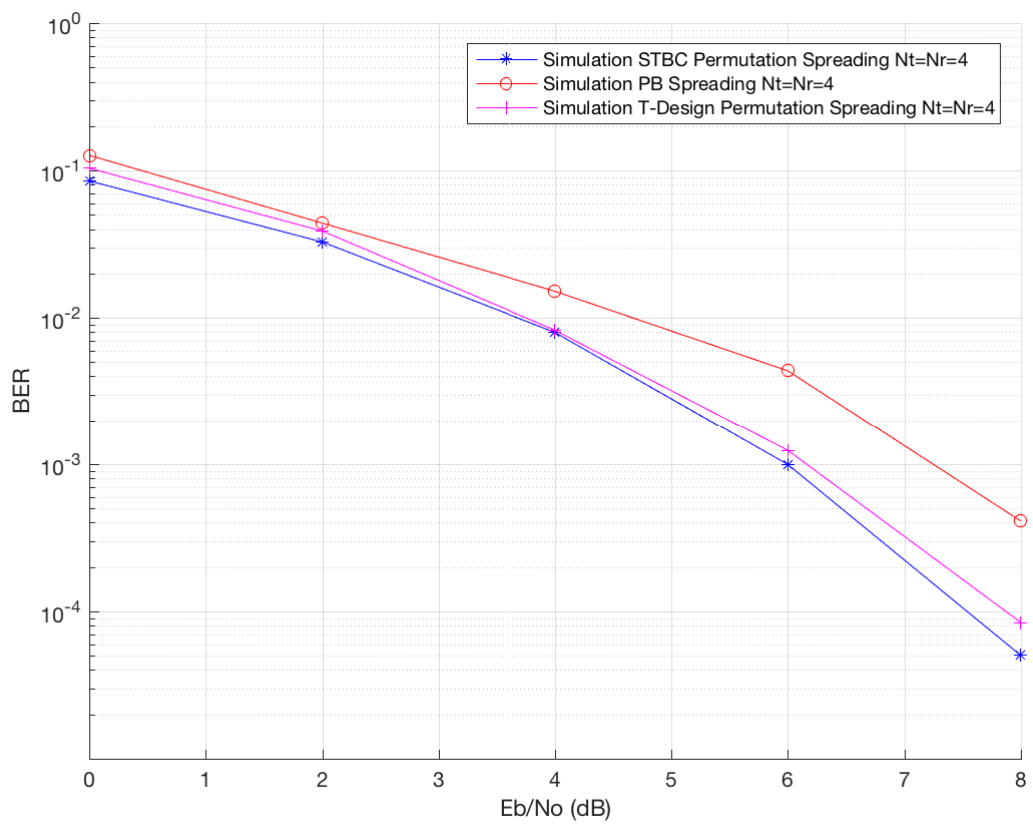

Figure 2.8: BER performance comparison of MIMO-CDMA System using the three techniques 


\subsubsection{Discussion}

It can be seen from Figures 2.7 and 2.8 that permutation methods provide better BER performance compared to the parity bit selected spreading technique. This is due to the difference in the decision variables of each method. In the case of the parity bit selected spreading, only one decision variable per antenna contains the sum of the transmitted signals plus noise; all the other decision variables contain only noise. However, in permutation spreading all decision variables contain one signal component plus noise or only noise. Moreover, the permutation spreading using STBC design provides slightly improvement in the bit error rate (BER) performance comparing to T-design without any increase of the system complexity. This is due to the fact that the STBC design adds certain code symmetry, which adds some degree of freedom in the Euclidean distance between constellation points [25]. Finally, We can notice that the union bound estimation is close to the

simulation results and it is tight as the SNR increases. This is due because the union bound estimation doesn't include the probability of intersection events which tends towards zero as the SNR increases. 


\section{Chapter 3}

\section{Spatial Modulation}

\subsection{Introduction}

Spatial modulation (SM) is a spatial multiplexing transmission technique designed for MIMO wireless systems. It was first proposed in [30] with the main goal to increase the spectral efficiency and reduce the inter-channel interference (ICI) effect. This is achieved by combining the spatial position of transmit antennas and conventional digital modulation schemes. The basic idea behind the Spatial Modulation is to activate only one transmit antenna at each signaling interval. The position (index) of the activated transmit antenna depends on the incoming data bits and, therefore, this can be used as an additional modulation scheme.

As shown in Figure 3.1 [32], the incoming data bits are divided into two groups. The first group is mapped into a constellation symbol in the signal domain using conventional modulation schemes, and the second group is mapped into a constellation symbol in the spatial domain using the antenna index [31].

One additional benefit of Spatial Modulation (SM) is the reduction of the cost and complexity of MIMO systems, as only one radio frequency (RF) chain is needed at the transmitter. This is because only one transmit antenna is activated at each time instant.

It is shown in [30] [31] that Spatial Modulation (SM) can achieve a spectral efficiency of 


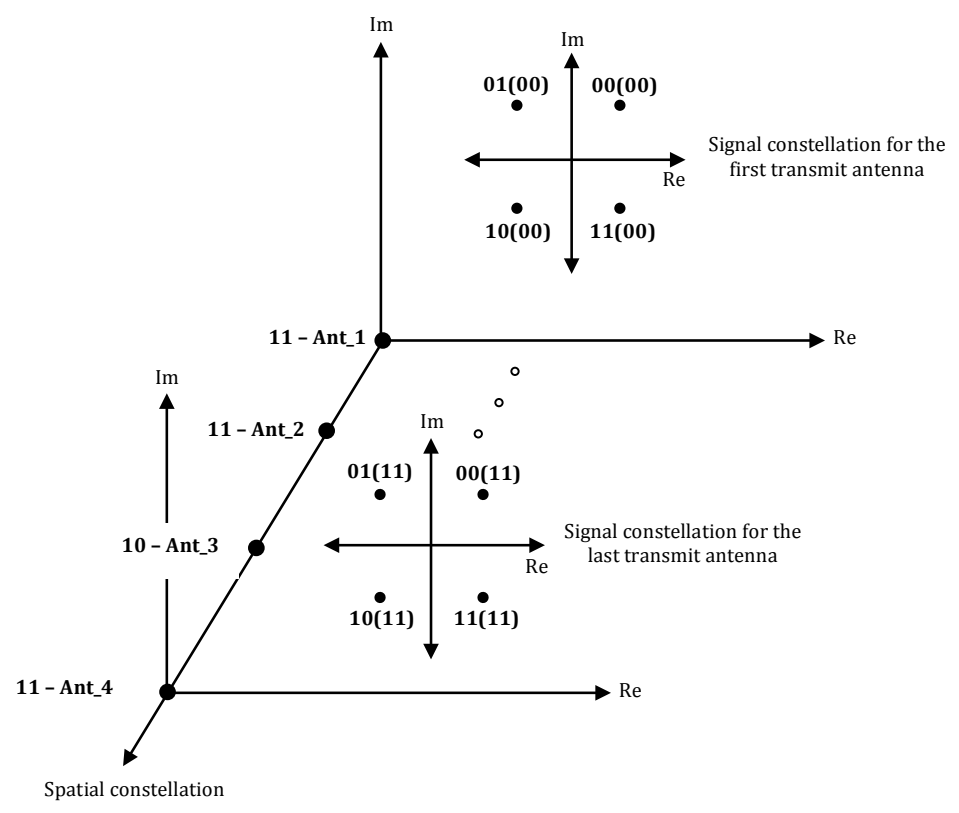

Figure 3.1: SM constellation diagram

$\log _{2}\left(N_{t}\right)$, where $N_{t}$ is the number of transmit antennas, compared to V-BLAST. However, this logarithmic increase will require a large number of available antennas ${ }^{1}$. In order to overcome this limitation, an extended technique called Generalized Spatial Modulation (GSM) is proposed in [33] where more than one transmit antenna is activated at each signaling interval. In GSM, data bits are mapped into different combinations of transmit antennas at each time instant. Furthermore, GSM technique provides additional spatial diversity gain, which improves the reliability of the communication channel.

Motivated by the above goal, various versions of Spatial Modulation (SM) were proposed. In [34] a method based on Space Shift Keying (SSK) termed as General SSK (GSSK) is presented where more than one antenna is activated at each signaling interval and all data bits are mapped into the activated antennas. Hence, no signal constellation is used. Simulations in [34] showed that GSSK requires less number of transmit antennas to achieve the same performance as SM. Another technique called Fractional Bit Encoded Spatial Modulation (FBE-SM) is proposed in [35] where the theory of modulus conversion is used. In this case, an arbitrary number of transmit antennas can be used. One of the

\footnotetext{
${ }^{1}$ number of transmit antenna should be a power of two.
} 
main drawbacks of this method is the error propagation. As discussed in [33], GSM is proven to provide the same performance, with less required transmit antennas, as SM, FBE-SM and GSSK. In this section, only the conventional SM and GSM methods are discussed.

Figure 3.2 illustrates the difference between the concept of spatial modulation (SM), Orthogonal Space Time Block Coding (OSTBC) and Spatial Multiplexing (SMX) [9].

It can be seen from Figure 3.2 that, in the case of SMX, the two symbols S1 and S2 are transmitted at the same time instance using both antennas with a rate of $N_{t} *$ $\log _{2}(M)$, where $\mathrm{M}$ is the modulation order and $N_{t}$ is the number of transmit antennas. For OSTBC, the two symbols are first encoded using Alamouti algorithm, then transmitted in two signaling interval and using both transmit antennas. The data rate for OSTBC is $N_{m} / N_{c u} * \log _{2}(M)$, where $N_{c u}$ is the number of channel use and $N_{m}$ is the number of transmitted symbols. In the other hand, SM uses one symbol (S2) to select the transmit antenna to activate and transmit the second symbol (S1) using this transmit antenna. The data rate in this case is $\log _{2}\left(N_{t}\right)+\log _{2}(M)$.

\subsection{Spatial Modulation (SM) system model}

The system model of the Spatial Modulation (SM) is shown in Figure 3.3 below where 4 transmit antennas and 4 received antennas are used [36]. In this system, BPSK is considered for illustration purpose. Different constellation schemes and number of antennas can, also, be used as described in details in [30].

\subsubsection{Transmitter Model}

At the transmitter side, the incoming information bits $\boldsymbol{q}(n)$ are, first, divided into subgroups of length $m=\log _{2}\left(N_{t}\right)+\log _{2}(M)$, where M is the QAM constellation order and $N_{t}$ is the number of transmit antennas. The matrix $\boldsymbol{x}(n)$ is generated by grouping, in columns, the subgroups of the incoming bits $\boldsymbol{q}(n)$. The next step is the spatial modulation mapping 

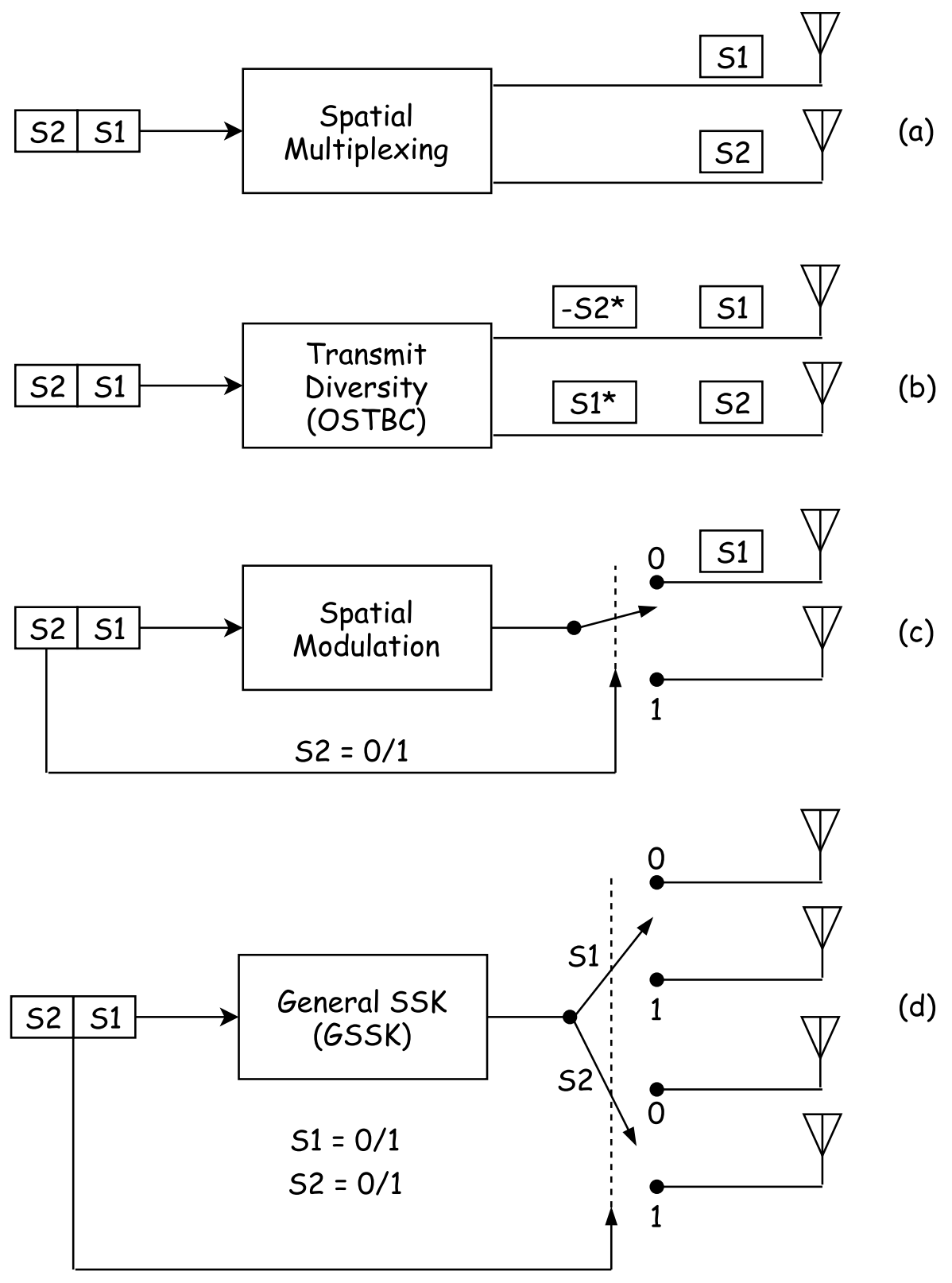

Figure 3.2: Working concepts of (a) SMX, (b) OSTBC, (c) SM and (d) GSSK 


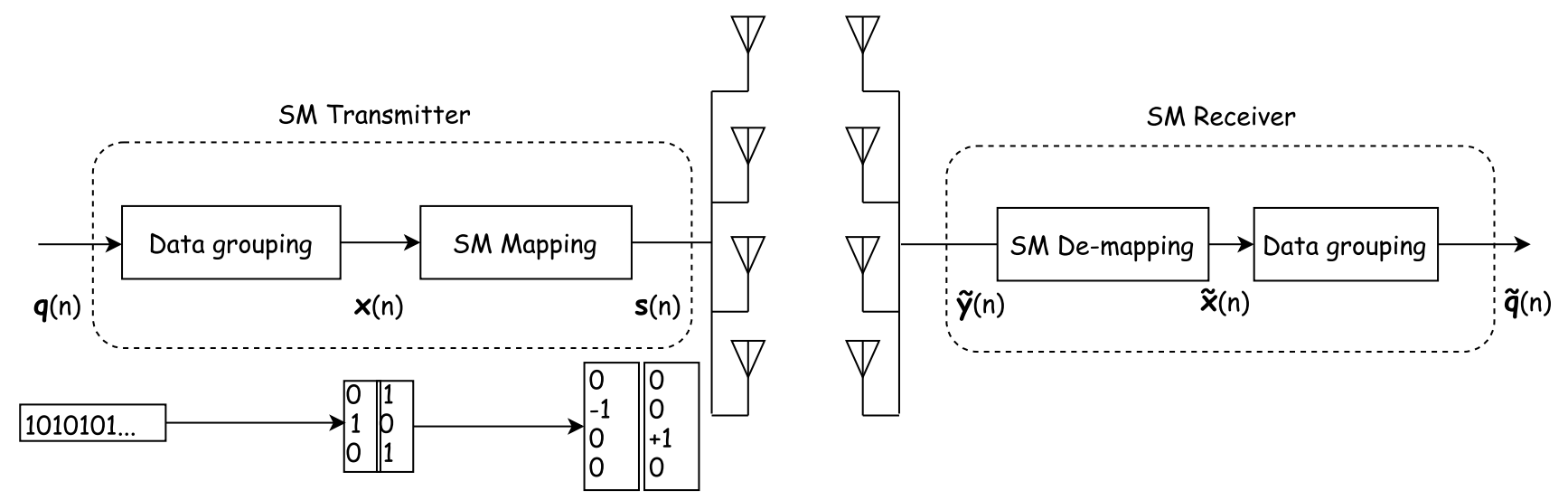

Figure 3.3: Spatial Modulation system model

where the vector $\boldsymbol{x}(n)$ is mapped to a new matrix $\boldsymbol{s}(n)$ using the mapping Table 3.1. As shown in Figure 3.3, the matrix $\boldsymbol{s}(n)$ contains one nonzero element in each column which is the symbol to transmit, at each time instance, using only one transmit antenna. An example of this process is shown in Figure 3.3 for two time instants. At the first signaling interval, the data to transmit is $\boldsymbol{x}(1)=\left[\begin{array}{lll}0 & 1 & 0\end{array}\right]^{T}$ that is mapped, using the mapping table, to $\boldsymbol{s}(1)=\left[\begin{array}{llll}0 & -1 & 0 & 0\end{array}\right]$. In this case, only the second antenna will be active and will be transmitting the symbol $s=-1$ and all other antennas will be silent.

\begin{tabular}{|c|c|c|}
\hline $\begin{array}{c}\text { Grouped } \\
\text { Bits }\end{array}$ & $\begin{array}{c}\text { Antenna } \\
\text { Index }\end{array}$ & Symbol \\
\hline 000 & 1 & -1 \\
001 & 1 & +1 \\
010 & 2 & -1 \\
011 & 2 & +1 \\
100 & 3 & -1 \\
101 & 3 & +1 \\
110 & 4 & -1 \\
111 & 4 & +1 \\
\hline
\end{tabular}

Table 3.1: SM mapping table using BPSK and Nt=4

Other modulation scheme can be used in order to increase the data rate. For instance, four transmit antennas with 4QAM modulation can be used to transmit 4 data bits as shown in the mapping Table 3.2 below. In this example, the first two bits are used to 
select the antenna to activate and the second two bits are mapped to a constellation symbol selected based on the 4QAM modulation.

\begin{tabular}{|c|c|c|c|c|c|}
\hline $\begin{array}{c}\text { Input } \\
\text { Bits }\end{array}$ & $\begin{array}{c}\text { Antenna } \\
\text { Index }\end{array}$ & $\begin{array}{c}\text { Transmit } \\
\text { Symbol }\end{array}$ & $\begin{array}{c}\text { Input } \\
\text { Bits }\end{array}$ & $\begin{array}{c}\text { Antenna } \\
\text { Index }\end{array}$ & $\begin{array}{c}\text { Transmit } \\
\text { Symbol }\end{array}$ \\
\hline 0000 & 1 & $+1+j$ & 1000 & 3 & $+1+j$ \\
0001 & 1 & $-1+j$ & 1001 & 3 & $-1+j$ \\
0010 & 1 & $-1-j$ & 1010 & 3 & $-1-j$ \\
0011 & 1 & $+1-j$ & 1011 & 3 & $+1-j$ \\
0100 & 2 & $+1+j$ & 1100 & 4 & $+1+j$ \\
0101 & 2 & $-1+j$ & 1101 & 4 & $-1+j$ \\
0110 & 2 & $-1-j$ & 1110 & 4 & $-1-j$ \\
0111 & 2 & $+1-j$ & 1111 & 4 & $+1-j$ \\
\hline
\end{tabular}

Table 3.2: SM mapping table using QPSK and $\mathrm{Nt}=4$

\subsubsection{Receiver model}

At the receiver, the index of the activated transmit antenna and the transmitted symbol are estimated using different algorithms such as Maximum Receiver Ratio Combining (MRCC) described in [30] and Maximum Likelihood (ML) described in [37]. This information is then used to retrieve the transmitted data bits using the same mapping table at the transmitter. The received signal is expressed as follow:

$$
\boldsymbol{Y}=\boldsymbol{H} \boldsymbol{S}+\boldsymbol{N}
$$

where $\boldsymbol{Y}$ is $N_{r}$ x 1 received matrix, $\boldsymbol{N}$ is the $N_{r}$ x 1 complex additive white noise (AWGN) matrix. $\boldsymbol{H}$ is the $N_{r} \times N_{t}$ channel gain vector and $\boldsymbol{S}$ is $N_{t}$ x 1 transmitted signal matrix.

One of the receiver algorithms used in [36] to estimate the transmitted data bit is the ML. As described in [36], the ML compares the received vector with all possible transmitted combinations and selects the option that provides the minimum Euclidian distance. Thus, the following formula applies in order to estimate the complex symbol $(\hat{s})$ and the antenna index $(\hat{p})$ : 


$$
(\hat{p}, \hat{s})=\operatorname{argmin}_{p, s} \sum_{i=1}^{N_{r}}\left\|y_{i}-H_{p, s} . s\right\|^{2}
$$

where $y_{i}$ is the received vector at the $i t h$ received antenna when transmitting symbol $s$ and using the $p t h$ antenna with $1 \leq p \leq N_{t}$ and $s \in\{M\}$.

Equation 3.2 requires high computational complexity $O\left(N_{t} M\left(4 N_{r}-1\right)\right)$, that increases linearly with the number of transmit antennas. This is due because the receiver needs to evaluate all possible combinations. To solve this issue a low-complexity detector, based on Sphere Decoding (SD) and tree search structure, is proposed in [36]. The SD aims to decrease the receiver computational complexity by applying ML detection over all points that are inside a sphere of a specific radius $\mathrm{C}$. The proposed algorithm combines the concept of the conventional Sphere Decoder, described in [36], and the tree search structure in order to avoid the main problem of having no point inside the sphere. Moreover, in this new method the computational complexity of the points inside the sphere is less then the conventional SD because only one transmit antenna is activated at each time instant[38].

The algorithm, first, sets an initial radius that depends on the noise level at the receiver as given in 3.3. It, then, searches for any point that has a Euclidean distance less or equal to the fixed radius C. Next, the algorithm updates the radius value by the Euclidean distance of the founded point. Finally, the point with the minimum Euclidean distance is considered to be the solution

$$
C^{2}=2 \alpha N_{r} \sigma_{n}^{2}
$$

Where $\sigma_{n}^{2}$ is the variance of the noise at the signaling interval $n$ and $\alpha$ is a constant used to maximize the possibility of having transmitted constellation point inside the used sphere.

Simulations in [36] showed that the proposed Sphere Decoder (SD) could achieve the same performance as the ML with a reduction of the computational complexity up to $85 \%$. 


\subsection{Generalized Spatial Modulation (GSM) system model}

Generalized Spatial Modulation (GSM) was first introduced in [33] and it is an extension of SM technique described in the pervious section. It was developed in order to overcome the limitation of the logarithmic increase of the number of transmit antennas in SM. As oppose to SM, in GSM more than one transmit antenna can be activated at each signaling interval and the same complex symbol is transmitted over the activated transmit antennas. Therefore, the antennas combinations are used as spatial constellation points to increase the spectral efficiency. In GSM, the number of transmit antennas combinations that can

be used should be a power of two and the total number of possible combinations is $\left(\begin{array}{c}N_{t} \\ N_{a}\end{array}\right)$, where $N_{a}$ is the number of antenna to activate at each time instant and $N_{t}$ is the number of available transmit antennas. As discussed before, SM has a spectral gain of $\log _{2}\left(N_{t}\right)$. Whereas in GSM the spectral gain is $\left\lfloor\log _{2}\left(\begin{array}{c}N_{t} \\ N_{a}\end{array}\right)\right\rfloor$, where $\lfloor$.$\rfloor is the floor operation. It$ can be noticed that GSM has greater spectral gain than SM for the same number of transmit antennas. Moreover, in Generalized Spatial Modulation the receiver receives multiple copies of the transmitted symbol, which provides additional diversity gain.

The Generalized Spatial Modulation system model is shown in Figure 3.4 [33]. In this example, 5 transmit antennas are used where only two can be activated at each signaling interval.

\subsubsection{Transmitter Model}

The incoming data bits $\boldsymbol{q}(\boldsymbol{n})$ are, first, divided into subgroups of length $m=m_{a}+m_{s}=$ $\left\lfloor\log _{2}\left(\begin{array}{c}N_{t} \\ N_{a}\end{array}\right)\right\rfloor+\log _{2}(M)$. The first $m_{a}$ bits, of each subgroups, are used to select the transmit antennas combination from Table 3.3. The next $m_{s}$ bits are modulated using any signal modulations schemes such as M-QAM.

For example, it can be seen from Figure 3.4 that the data bits to be transmitted at the

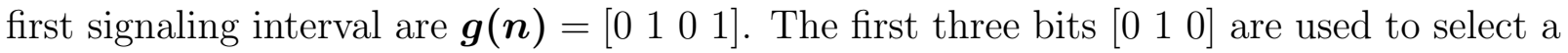




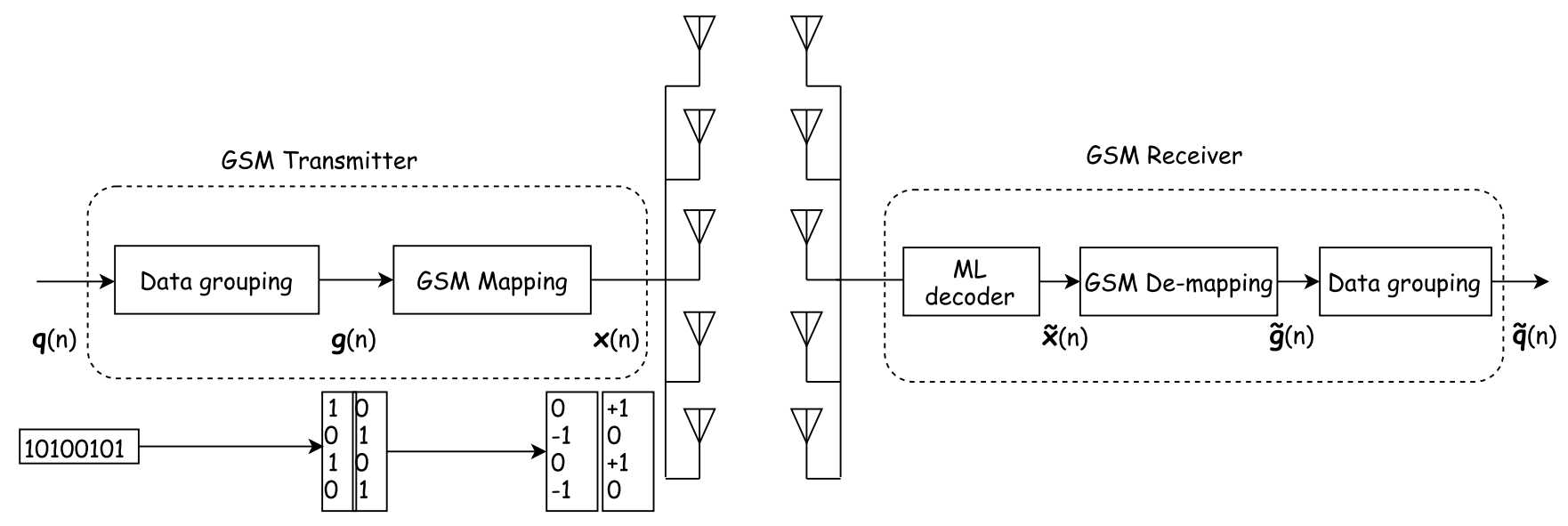

Figure 3.4: Generalized Spatial Modulation system model

combination of transmit antenna from Table 3.3, which are antennas 1 and 4 . Then, the last bit $\{1\}$ is modulated using BPSK, which is equivalent to a symbol $s=+1$. Therefore, the corresponding column of the mapped vector at this time instant is $x(n)=\left[\begin{array}{lllll}+1 & 0 & 0 & +1 & 0\end{array}\right]$. For the same example, if the SM is used, the required number of transmit antennas is equal to eight in order to keep the same spectral efficiency.

Other modulation scheme can be used in order to increase the number of transmitted bits. For instance, in the example shown in Table 3.3 the data bit can be increased to 5 bits by using QPSK modulation.

Motivated by the same concept of GSM, a modified version termed as Variable Generalized Spatial Modulation (VGSM) is proposed in [39] where the number of transmit antennas to activate, at each signaling interval, varies from one transmit antenna to more than one. The aim of this method is to increase the number of combinations that can be used. In VGSM, the total number of possible combinations is expressed as follows:

$$
\rho_{V G S M}=\sum_{i=1}^{N_{t}}\left(\begin{array}{c}
N_{t} \\
i
\end{array}\right)=2^{N_{t}}-1
$$

Same as GSM, the number of possible combinations to use should be a power of two. Therefore, the spectral efficiency is expressed as follows: 


\begin{tabular}{|c|c|c|}
\hline Group Bits & Antenna Combination $(l)$ & Symbol $(s)$ \\
\hline 0000 & $(1,2)$ & -1 \\
0001 & $(1,2)$ & +1 \\
0010 & $(1,3)$ & -1 \\
0011 & $(1,3)$ & +1 \\
0100 & $(1,4)$ & -1 \\
0101 & $(1,4)$ & +1 \\
0110 & $(1,5)$ & -1 \\
0111 & $(1,5)$ & +1 \\
1000 & $(2,3)$ & -1 \\
1001 & $(2,3)$ & +1 \\
1010 & $(2,4)$ & -1 \\
1011 & $(2,4)$ & +1 \\
1100 & $(3,5)$ & -1 \\
1101 & $(3,5)$ & -1 \\
1110 & $(4,5)$ & +1 \\
1111 & $(4,5)$ & \\
\hline
\end{tabular}

Table 3.3: GSM mapping table using BPSK, $\mathrm{Nt}=5$ and $\mathrm{Na}=2$

$$
\eta_{V G S M}=\left\lfloor\log _{2}\left(2^{N_{t}}-1\right)\right\rfloor+\log _{2}(M)
$$

To illustrate this concept, an example is shown in Table 3.4 where 4 transmit antennas are used. It can be seen that 3 bits can be mapped into different combinations using VGSM. However, if SM or GSM are used with the same number of transmit antennas, only 2 bits can be mapped to the transmit antennas combinations.

\subsubsection{Receiver Model}

The same decoding algorithms as SM can be used to estimate the transmitted complex symbol and the transmit antenna combination used at the transmitter. The received signal is given by:

$$
\boldsymbol{Y}=\boldsymbol{H} \boldsymbol{S}+\boldsymbol{N}
$$

where $\boldsymbol{Y}$ is $N_{r}$ x 1 received matrix, $\boldsymbol{N}$ is the $N_{r}$ x 1 complex additive white noise (AWGN) matrix. $\boldsymbol{H}$ is the $N_{r}$ x $N_{t}$ channel gain vector and $\boldsymbol{S}$ is $N_{t}$ x 1 transmitted signal 


\begin{tabular}{|c|c|}
\hline Group Bits & Antenna Combination $\left(l_{\text {VGSM }}\right)$ \\
\hline 000 & $(1)$ \\
001 & $(2)$ \\
010 & $(3)$ \\
011 & $(4)$ \\
100 & $(1,2)$ \\
101 & $(1,3)$ \\
110 & $(1,4)$ \\
111 & $(2,3)$ \\
\hline
\end{tabular}

Table 3.4: VGSM mapping table using $\mathrm{Nt}=4$

matrix.

ML is used to estimate the complex symbol $(\hat{s})$ and the spatial symbol $(\hat{p})$ as follows:

$$
(\hat{p}, \hat{s})=\operatorname{argmin}_{p, s} \sum_{i=1}^{N_{r}}\left\|y_{i}-H_{p, s} . s\right\|^{2}
$$

where $y_{i}$ is the received message at the $i t h$ received antenna when transmitting symbol $s$ and using the pth antenna with $1 \leq p \leq N_{t}$ and $s \in\{M\}$.

\subsection{Receiver complexity}

The receiver complexity can be computed based on the number of complex operations needed by the detection algorithm. As discussed before, the evaluation of the decision variable, for the spatial modulation using ML, in Equation 3.2 can be expressed as follows [36]:

$$
C_{S M_{M L}}=N_{t} M\left(4 N_{r}-1\right)
$$

On the other hand, the GSM receiver complexity using ML can be expressed as follow [33]:

$$
C_{G S M_{M L}}=N_{r} N_{t} M\left(N_{a}+2\right)
$$

The complexity ratio between these two techniques can be computed as follows: 


$$
R=\frac{C_{G S M_{M L}}}{C_{S M_{M L}}}=\frac{N_{r} N_{t} M\left(N_{a}+2\right)}{N_{t} M\left(4 N_{r}-1\right)}=\frac{N_{r}\left(N_{a}+2\right)}{\left(4 N_{r}-1\right)}
$$

Figure 3.5 illustrates the variation of this ratio in terms of the activated antennas $N_{a}$ for $N_{r}=4$. As shown in the Figure 3.5, the GSM receiver complexity increases as the number of transmit antennas to activate $N_{a}$ increases. For instance, to transmit four bits using BPSK the number of transmit antennas, using GSM, is $N_{t}=5$ with $N_{a}=2$. This corresponds to an increase of $6 \%$ of the complexity comparing to SM. However, this increase is compensated by the reduction of the number of total transmit antennas needed. For the same example, the number of total transmit antennas needed for SM is $N_{t}=8$.

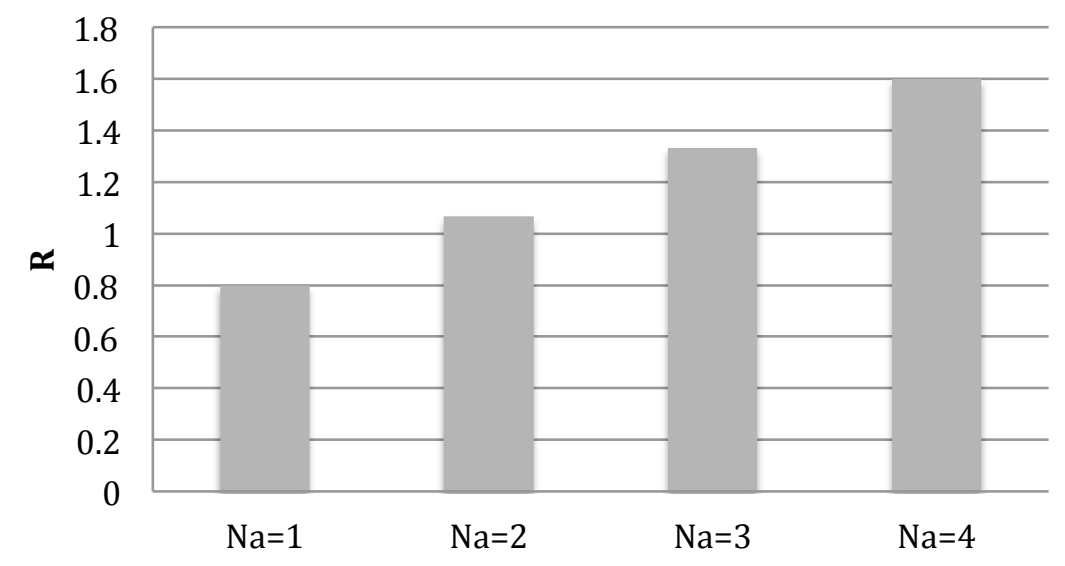

Figure 3.5: Comparison of receiver complexity between SM and GSM using ML detection

\subsection{Simulation results and discussion}

This section discusses the simulation results of different Spatial Modulation configurations. All simulations are performed using Matlab where the following assumption are considered:

- All simulations are performed over Rayleigh flat-fading channels.

- All channel gains are considered to be independent, frequency non-selective and slowly varying complex Gaussian random variables with zero means and unit variance. 
- Perfect channel state information (CSI) is considered to be available at the receiver with zero power penalty.

- BPSK modulation is used for all discussed methods and data bits are generated such a way to ensure at least 100 errors counted. All configurations have four receiver antennas.

Three scenarios are considered in order to achieve a spectral efficiency of $\eta=4 b i t s / s / H z$, $\eta=6 b i t s / s / H z$ and $\eta=8 b i t s / s / H z$. Figure 3.6 shows the BER performance using Spatial Modulation of the three scenarios. It can be seen that as the number of bits to transmit increases, the number of transmit antennas increases as well, which increase the complexity and thus degrades the SM performance. In Figure 3.7 a comparison of BER performance for $\eta=4 b i t s / s / H$ is shown, where $N_{t}=8$ for SM, $N_{t}=5$ and $N_{a}=2$ for GSM and $N_{t}=4$ for VGSM. The performances of the three techniques are close to each other with a slightly better BER of SM at high SNR. However, GSM and VGSM require about half the number of transmit antennas compared to SM. For $\eta=6 b i t s / s / H$ results are shown in Figure 3.8, where $N_{t}=32$ for SM, $N_{t}=7$ and $N_{a}=3$ for GSM and $N_{t}=6$ for VGSM. Figure 3.9 illustrates results for $\eta=8 b i t s / s / H$, where $N_{t}=128$ for SM, $N_{t}=12$ and $N_{a}=3$ for GSM and $N_{t}=8$ for VGSM. Same observation can be noticed in both figures where SM has slightly better performance at high SNR. Moreover, it can be seen that VGSM offers slightly better results than GSM with less required transmit antennas.

In summary, GSM and VGSM can provide acceptable performance, comparing to SM, with less number of transmit antennas and less complexity. 


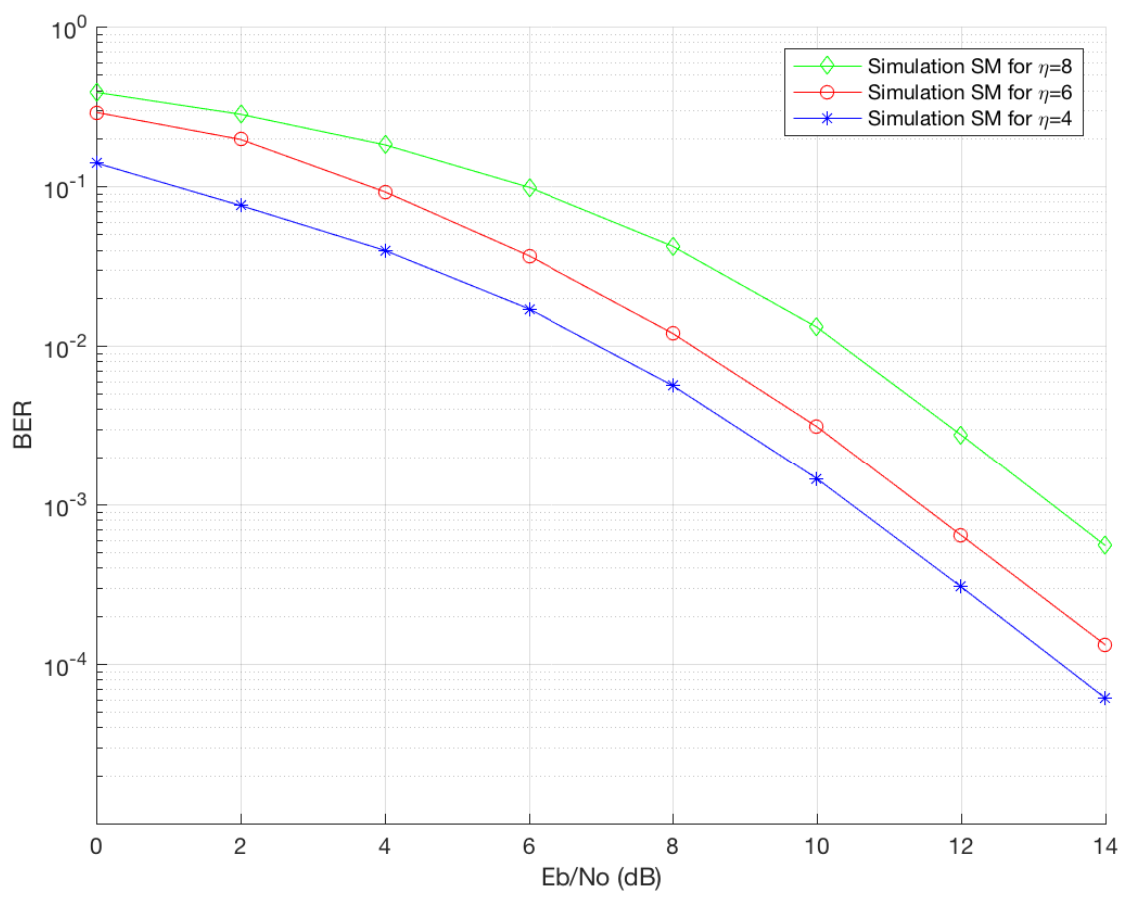

Figure 3.6: Comparison of SM performances, where $N_{t}=128$ for $\eta=8$ bits, $N_{t}=32$ for $\eta=6 b i t s, N_{t}-8$ for $n-1$ hits ucins RDCK mndulntinn and $N T-1$

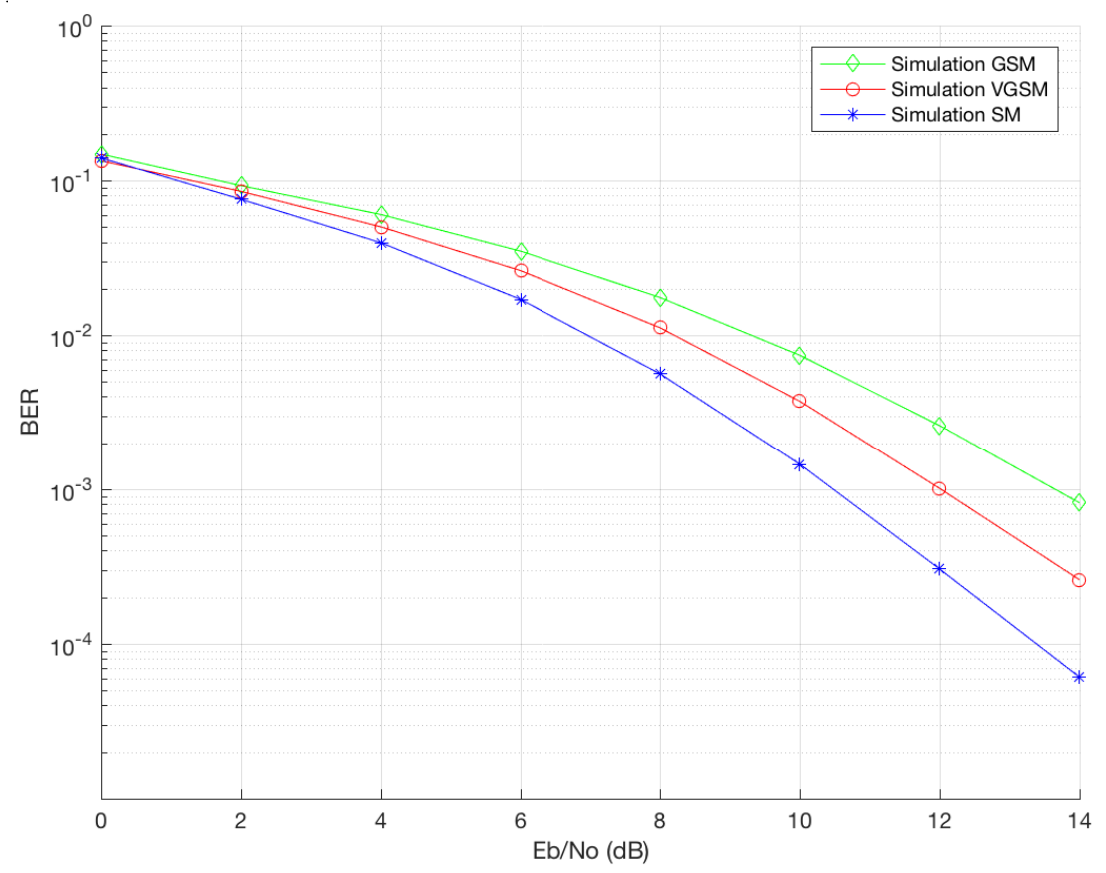

Figure 3.7: BER performance of SM, GSM and VGSM where $\eta=4 b i t s, N_{r}=4$ and using BPSK modulation. 


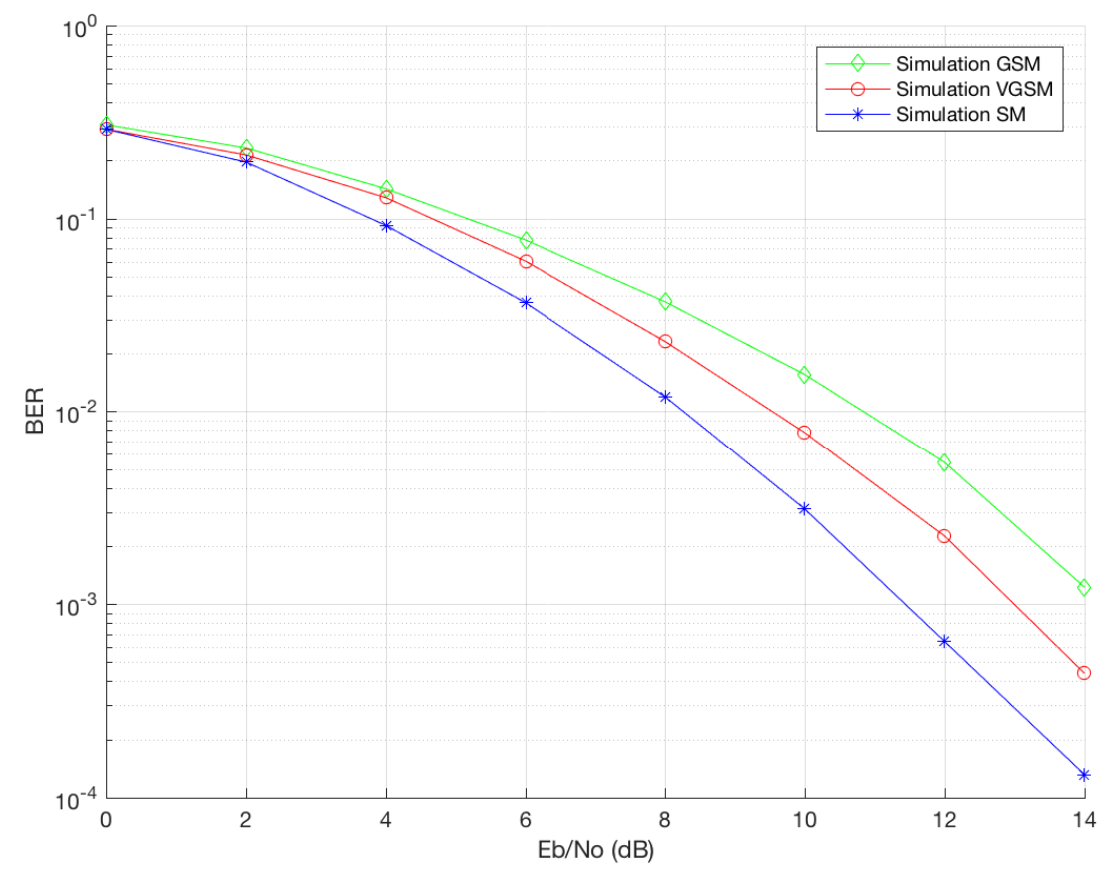

Figure 3.8: BER performance of SM, GSM and VGSM where $\eta=6 b i t s, N_{r}=4$ and using BPSK modulation.

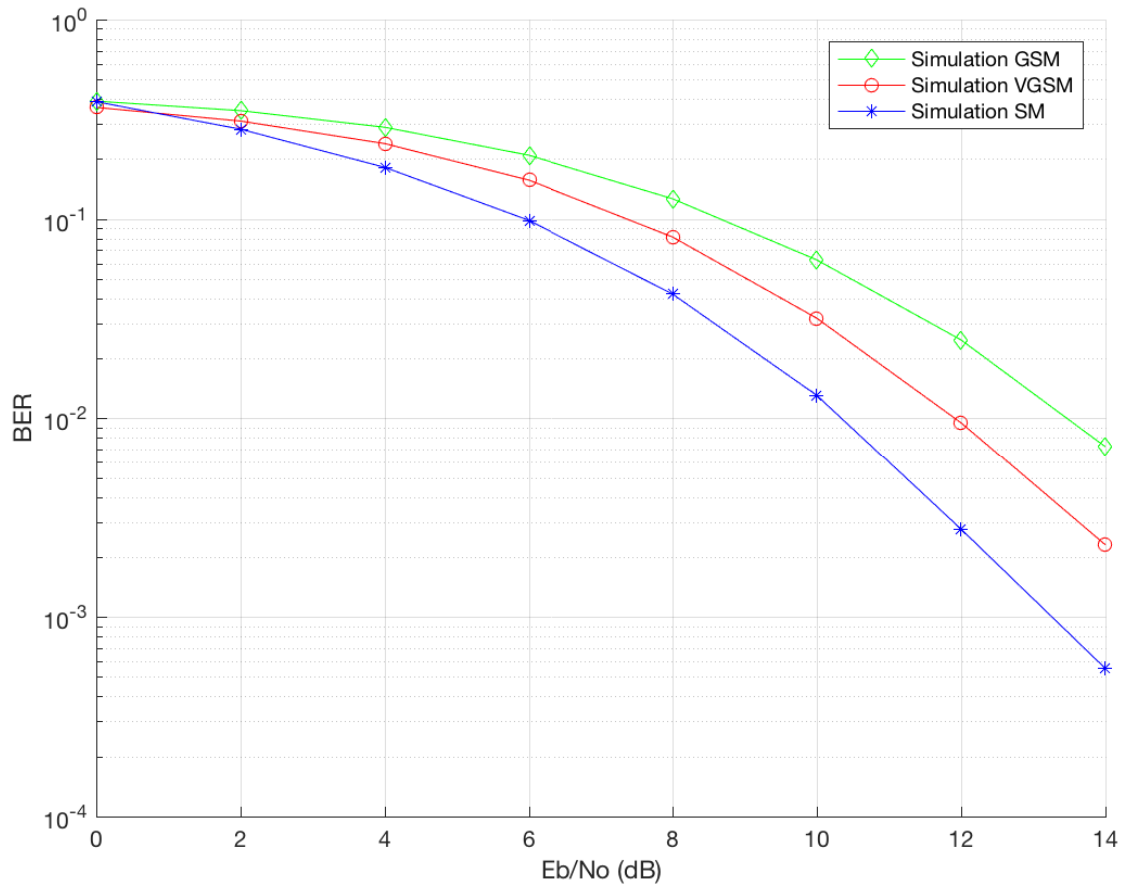

Figure 3.9: BER performance of SM, GSM and VGSM where $\eta=8 b i t s, N_{r}=4$ and using BPSK modulation. 


\subsubsection{Discussion}

This chapter introduced the concept of SM and how the spatial positions of transmit antennas can be used as additional information that can increase the spectral efficiency of MIMO systems. The chapter also discussed the generalized idea of Spatial Modulation (GSM) where more than one transmit antennas can be activated to send same data at each signaling interval. GSM aims to overcome the limitation of SM where a power of two transmit antennas is needed. It also offers additional spatial diversity gain. Then, the chapter introduced an extension method of GSM where the number of transmit antennas to activate, at each signaling interval, varies from one transmit antenna to more than one. This techniques provides higher number of transmit antennas combinations compared to GSM. Finally, Simulation results showed that the performance of these three techniques is close to each other with slightly better BER performance of SM. However, the VGSM and GSM can achieve acceptable performance with less transmit antennas and less complexity compared to SM. 


\section{Chapter 4}

\section{Single User Permutation Spreading Employing Spatial Modulation for MIMO-CDMA system}

\subsection{Introduction}

The application of SM has been discussed in different studies and it has been shown that it can increase the spectral efficiency while avoiding Inter-Channel Interference (ICI) [30]. Moreover, as explained in chapter 2, SM reduces the hardware system cost, complexity and energy consumption by using less Radio Frequency (RF) chains compared to conventional MIMO systems.

On other hand, the extension of parity bit selected spreading technique to MIMOCDMA systems which was introduced in [22] and discussed in chapter 2, shows that a performance gain can be obtained when the selected spreading sequences depends on the data that is to be transmitted. In this chapter we introduce, for the first time, a new technique that combines the benefits of SM and MIMO-CDMA systems that use permutation spreading. Rather than activating all $N_{t}$ transmit antennas at each signaling interval in a MIMO-CDMA system employing parity bit selected or permutation spreading, only $N_{a}$ transmit antennas are activated. Similar to SM concept, a combination of transmit 
antenna is selected from the $\left(\begin{array}{l}N_{t} \\ N_{a}\end{array}\right)$ available possibilities where the selected combination of transmit antennas depends on which coset the messages to transmit comes from. By doing so, a reduction of system complexity and Inter-Channel Interference effect can be achieved while keeping a certain diversity order compared to SM.

As it is shown later in this chapter, a significant improvement in the performance of MIMO-CDMA systems with permutation spreading can be achieved at low SNR values when SM is implemented.

\subsection{System Model}

The proposed system model is discussed in this section for MIMO-CDMA systems with $N_{t}$ transmit antennas and $N_{r}$ receiver antennas. Binary phase shift keying (BPSK) is considered for illustration purpose. Other constellation schemes and different number of antennas can be used in order to increase the data rate.

\subsubsection{Transmitter Model}

The transmitter block diagram of the proposed technique is shown in Figure 4.1 where a MIMO-CDMA system with $N_{t}$ transmit antennas and $N_{r}$ receiver antennas is considered. In this system, the binary information streams are first divided into blocks of $\mathrm{k}$ bits, where $k=\log _{2}(M) * N_{t}$ for an M-ary modulation scheme. Then, the message bits are converted into $N_{t}$ parallel streams and fed into the SM mapping block and the spreading code selector. Similar to the permutation spreading technique discussed in Chapter 2, the spreading code selector chooses, based on the input bits, a unique spreading sequences $\left(w_{1}(t), w_{2}(t), \ldots, w_{N_{t}}(t)\right)$ from $N$ set of mutually orthogonal sequences $\left(c_{1}(t), c_{2}(t), \ldots, c_{N}(t)\right)$, where $N \geqslant N_{t}$. Additionally, the SM-mapping block selects the pair of antenna to activate and the information bits to transmit. Then, the message bits are modulated using BPSK and multiplied with the chosen spreading sequence before transmission. 


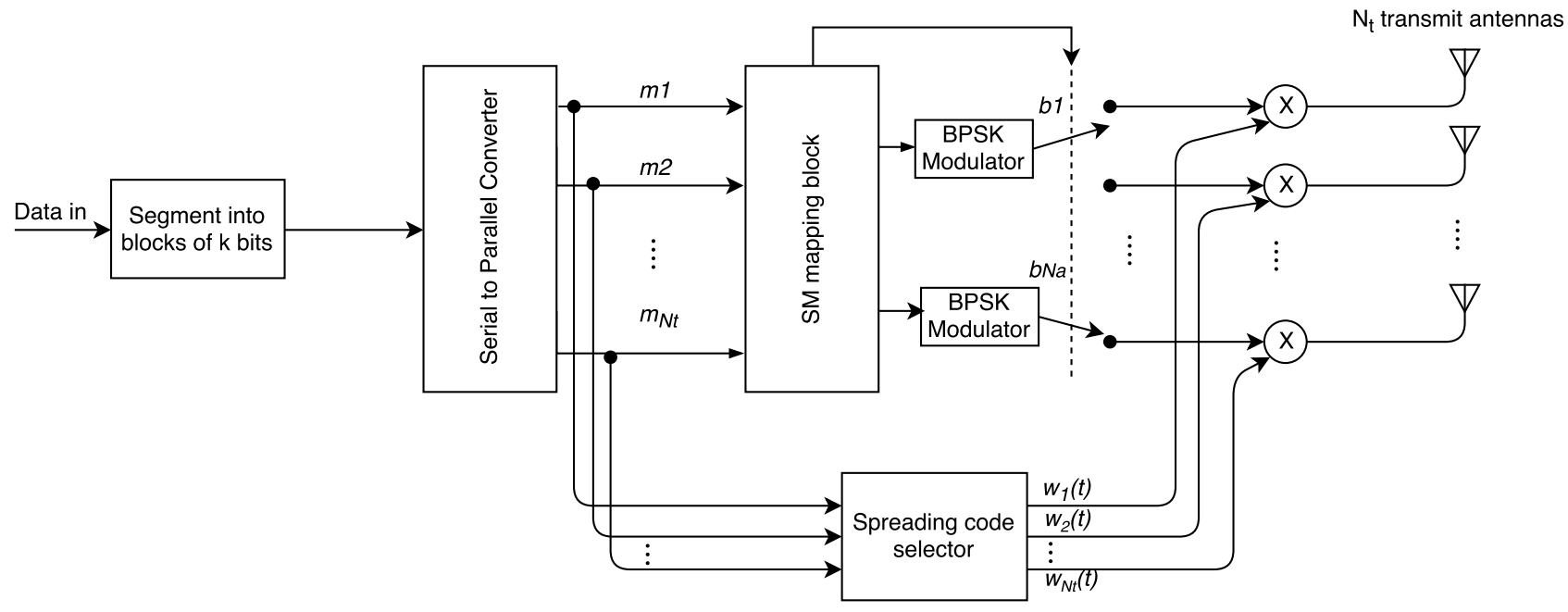

Figure 4.1: The Transmitter of MIMO-CDMA with permutation spreading using SM

The design strategy is similar to the permutation spreading method discussed in $[3,4]$ and introduced in chapter 2, but with some modifications. In the spreading code selector side, messages carried by the same spreading code are divided into cosets $\left(M_{1}, M_{2}, \ldots, M_{K}\right)$ so that the Euclidian distance between messages in each coset is maximized. The number of cosets $(K)$ depends on the number of antennas $\left(N_{t}\right)$ and the number of messages in each coset $(L): K=\left(2^{N_{t}}\right) / L$. Each coset will be assigned with a unique permutation spreading waveforms. If the parity bit selected spreading method is used, each coset will have the same spreading waveform that will be used by all transmit antennas.

The permutation design used is based on the Alamouti's orthogonal STBC matrix in order to preserve the orthogonality between message vectors and achieve a diversity order similar to STBC as shown in [25]. For a system with 4 transmit antennas, 8 orthogonal waveforms are needed, and thus the permutation table is generated based on the 8x8 STBC matrix shown in 4.1. One of possible spreading permutation table for such system is given as follows, where $w_{j}(t)$ refers to the spreading waveform assigned to antenna $j$ and $c_{i}(t)$ is the ith spreading code.

In the SM mapping block side, the message vector is divided into subgroups of length $m=m_{a}+m_{s}=\left\lfloor\log _{2}\left(\begin{array}{l}N_{t} \\ N_{a}\end{array}\right)\right\rfloor+\log _{2}(M) * N_{a}$, where $N_{a}$ is the number of transmit antenna to activate at each time instant and $\mathrm{Nt}$ is the number of available transmit antennas. The first 


\begin{tabular}{|c|c|c|c|c|c|}
\hline Coset & Message Vector & $\boldsymbol{w}_{\mathbf{1}}(\boldsymbol{t})$ & $\boldsymbol{w}_{\mathbf{2}}(\boldsymbol{t})$ & $\boldsymbol{w}_{\mathbf{3}}(\boldsymbol{t})$ & $\boldsymbol{w}_{\mathbf{4}}(\boldsymbol{t})$ \\
\hline$M_{1}$ & 0000 & $c_{1}(t)$ & $c_{2}(t)$ & $c_{3}(t)$ & $c_{4}(t)$ \\
\hline$M_{2}$ & $\begin{array}{l}0001 \\
1110\end{array}$ & $c_{2}(t)$ & $c_{1}(t)$ & $c_{4}(t)$ & $c_{3}(t)$ \\
\hline$M_{3}$ & 0010 & $c_{3}(t)$ & $c_{4}(t)$ & $c_{1}(t)$ & $c_{2}(t)$ \\
\hline$M_{4}$ & 1101 & $c_{4}(t)$ & $c_{3}(t)$ & $c_{2}(t)$ & $c_{1}(t)$ \\
\hline$M_{5}$ & 110011 & $c_{5}(t)$ & $c_{6}(t)$ & $c_{7}(t)$ & $c_{8}(t)$ \\
\hline$M_{6}$ & 0100 & $c_{6}(t)$ & $c_{5}(t)$ & $c_{8}(t)$ & $c_{7}(t)$ \\
\hline$M_{7}$ & 1011 & $c_{7}(t)$ & $c_{8}(t)$ & $c_{5}(t)$ & $c_{6}(t)$ \\
\hline$M_{8}$ & 10101 & $c_{8}(t)$ & $c_{7}(t)$ & $c_{6}(t)$ & $c_{5}(t)$ \\
\hline
\end{tabular}

Table 4.1: Permutation Spreading for MIMO-CDMA system with 4 transmit antennas Using STBC design

$m_{a}$ bits, of each subgroup, are divided into different cosets $\left(M_{1}^{\prime}, M_{2}^{\prime}, \ldots, M_{K^{\prime}}^{\prime}\right)$ that provide a maximum Euclidian distance between messages in each coset. The number of cosets $\left(K^{\prime}\right)$ depends on the number of antennas to activate $\left(N_{a}\right)$ and the number of messages in each coset $\left(L^{\prime}\right): K^{\prime}=\left(2^{N_{a}}\right) / L$. Each coset will be assigned with a unique pair of antenna to activate. The next $m_{s}$ bits are modulated using any signal modulations schemes such as M-QAM modulated and transmitted over the activated antennas.

One of possible SM mapping table for a MIMO system with four transmits antennas and four received antennas using BPSK modulation is shown in Table 4.2.

To illustrate this concept let us consider an example of MIMO-CDMA system with four transmit antennas and four receiver antennas as shown in Figure 4.2.

The first step in the design process consists of dividing the message vector into $K$ cosets with 2 messages per coset. In this case, 8 cosets are generated $M_{1}=\{0000 ; 1111\}$, $M_{2}=\{0001 ; 1110\}, M_{3}=\{0010 ; 1101\}, M_{4}=\{0011 ; 1100\}, M_{5}=\{0100 ; 1011\}, M_{6}=$ 


\begin{tabular}{|c|c|c|}
\hline Coset & Message Vector & Antenna Combination \\
\hline \multirow{2}{*}{$M_{1}^{\prime}$} & 00 & $(1,3)$ \\
\hline \multirow{2}{*}{$M_{2}^{\prime}$} & 11 & $(2,4)$ \\
\hline
\end{tabular}

Table 4.2: SM mapping table for MIMO system with four transmit antennas

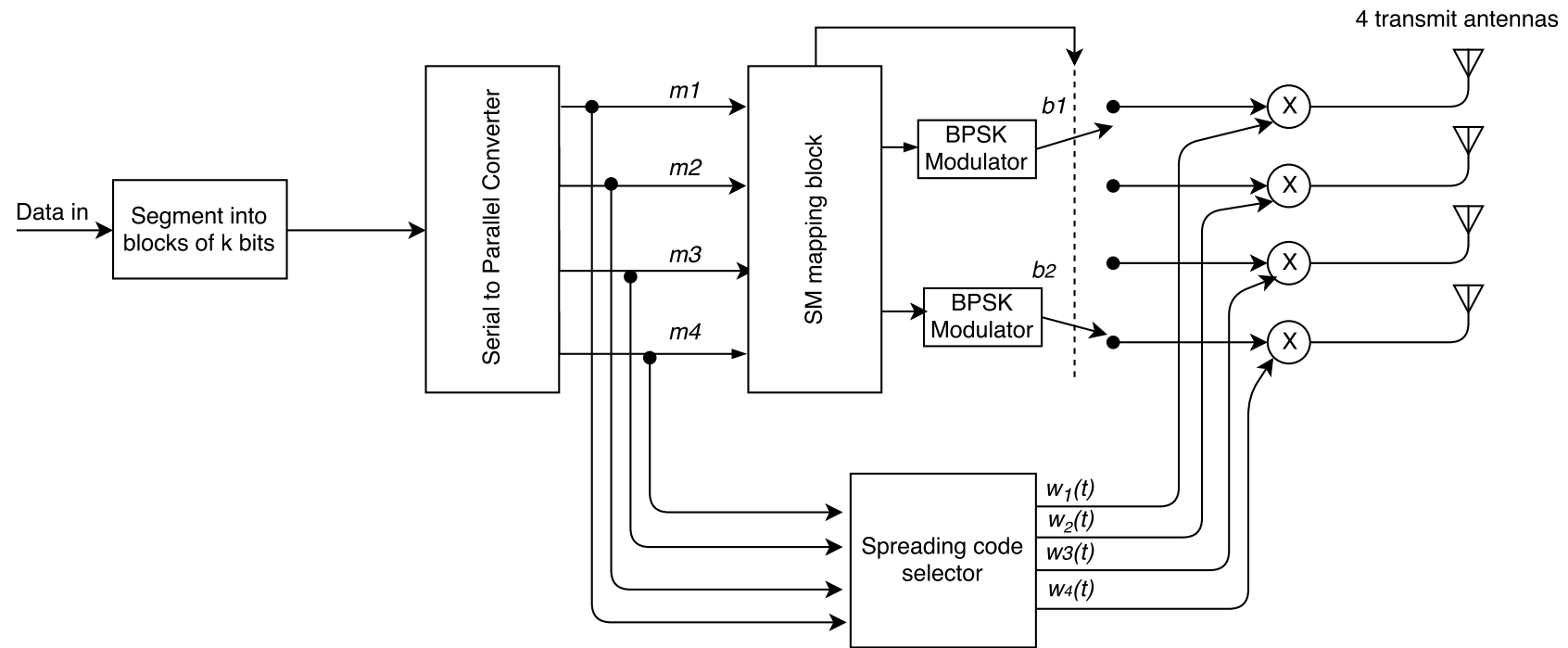

Figure 4.2: Transmitter for a 4x4 MIMO-CDMA with permutation spreading using SM

$\{0101 ; 1010\}, M_{7}=\{0110 ; 1001\}$ and $M_{8}=\{0111 ; 1000\}$. Each coset will have unique spreading sequences waveforms as shown in Table 4.1 .

In the next step, the message vector is divided into two sub-groups. The first sub-group is used to choose the combination of antennas to activate based on Table 4.2, and the second sub-group represent the final message bits to transmit after a BPSK modulation.

For example, if a message vector to be transmitted is $m=\left[\begin{array}{llll}0 & 1 & 1 & 1\end{array}\right]$ from coset $M_{8}$, then the transmit antennas to activate, based on table Table 4.2, are 2 and 4 and the final symbols to transmit are $\left[\begin{array}{ll}1 & 1\end{array}\right]$. Thus, the final transmit signals are given by: 


\begin{tabular}{|c|c|}
\hline Antenna1 & Deactivated \\
\hline Antenna 2 & $s_{2} * w_{2}(t)=c_{7}(t)$ \\
\hline Antenna3 & Deactivated \\
\hline Antenna 4 & $s_{4} * w_{4}(t)=c_{5}(t)$ \\
\hline
\end{tabular}

where $s_{j}$ is the symbol transmitted from the $j t h$ antenna; and the $w_{j}(t)$ is the spreading waveform assigned to antenna $j$.

Now, if the message vector to be transmitted is $m=\left[\begin{array}{llll}1 & 1 & 0 & 0\end{array}\right]$ from coset $M_{4}$, then the antennas to activate, based on Table 4.2, are 1 and 3 and the final symbols to transmit are $\left[\begin{array}{ll}-1 & -1\end{array}\right]$. Thus, the final transmit signals are given by:

\begin{tabular}{|c|c|}
\hline Antenna 1 & $s_{1} * w_{1}(t)=-c_{4}(t)$ \\
\hline Antenna 2 & Deactivated \\
\hline Antenna 3 & $s_{3} * w_{3}(t)=-c_{2}(t)$ \\
\hline Antenna 4 & Deactivated \\
\hline
\end{tabular}

The same idea can be applied to the parity bit selected spreading sequence where the only difference is that the transmit antennas use the same waveform at each signaling interval.

Table 4.3 shows the full transmit signal table for the system shown in Figure 4.2 using BPSK modulation.

\subsubsection{Receiver Model}

The proposed receiver model is shown in Figure 4.3. Similar to the permutation spreading sequences, each receiver antenna is equipped with a bank of matched filters that correspond to one of the $N$ spreading waveforms $\left(c_{1}(t), c_{2}(t), \ldots, c_{N}(t)\right)$. The receiver estimates the transmitted symbols and the index of the activated transit antennas using the ML and the output of the matched filters as decision variables. The ML compares the received vector with all possible transmitted combinations and selects the option that provides the 


\begin{tabular}{|c|c|c|c|c|c|}
\hline Coset & Message Vector & $w_{1}(t)$ & $w_{2}(t)$ & $w_{3}(t)$ & $w_{4}(t)$ \\
\hline \multirow{2}{*}{$M_{1}$} & 0000 & $-c_{1}(t)$ & $\ldots$ & $-c_{3}(t)$ & $\ldots$ \\
\hline & 1111 & $c_{1}(t)$ & $\ldots$ & $c_{3}(t)$ & $\ldots$ \\
\hline \multirow{2}{*}{$M_{2}$} & 0001 & $-c_{2}(t)$ & $\ldots$ & $c_{4}(t)$ & .... \\
\hline & 1110 & $c_{2}(t)$ & $\ldots$ & $-c_{4}(t)$ & $\ldots$ \\
\hline \multirow{2}{*}{$M_{3}$} & 0010 & $c_{3}(t)$ & $\ldots$ & $-c_{1}(t)$ & $\ldots$ \\
\hline & 1101 & $-c_{3}(t)$ & $\ldots$ & $c_{1}(t)$ & $\ldots$ \\
\hline \multirow{2}{*}{$M_{4}$} & 0011 & $c_{4}(t)$ & $\ldots$ & $c_{2}(t)$ & $\ldots$ \\
\hline & 1100 & $-c_{4}(t)$ & $\ldots$ & $-c_{2}(t)$ & $\ldots$ \\
\hline \multirow{2}{*}{$M_{5}$} & 0100 & $\ldots$. & $-c_{6}(t)$ & $\ldots$. & $-c_{8}(t)$ \\
\hline & 1011 & $\ldots$ & $c_{6}(t)$ & $\ldots$ & $c_{8}(t)$ \\
\hline \multirow{2}{*}{$M_{6}$} & 0101 & $\ldots$ & $-c_{5}(t)$ & $\ldots$ & $c_{7}(t)$ \\
\hline & 1010 & $\ldots$ & $c_{5}(t)$ & $\ldots$ & $-c_{7}(t)$ \\
\hline \multirow{2}{*}{$M_{7}$} & 0110 & $\ldots$ & $c_{8}(t)$ & $\ldots$ & $-c_{6}(t)$ \\
\hline & 1001 & $\ldots$ & $-c_{8}(t)$ & $\ldots$ & $c_{6}(t)$ \\
\hline \multirow{2}{*}{$M_{8}$} & 0111 & $\ldots$ & $c_{7}(t)$ & $\ldots$ & $c_{5}(t)$ \\
\hline & 1000 & $\ldots$ & $-c_{7}(t)$ & $\ldots$ & $-c_{5}(t)$ \\
\hline
\end{tabular}

Table 4.3: Permutation Spreading transmit table for 4x4 MIMO-CDMA system using SM

minimum Euclidian distance. This information is then used to retrieve the transmitted data bits using the same mapping table at the transmitter.

\section{Decision variable}

The decision variable for MIMO-CDMA system with permutation spreading sequences using SM is similar to the one discussed in chapter 2. However, in this case only pair of transmit antennas are used and therefore the system complexity is reduced.

Let us first consider MISO-CDMA system with $N_{t}$ transmit antennas and one receiver antenna where BPSK is used as modulation scheme. The received signal from a given message vector is the sum of the transmitted bits multiplied by the corresponding spreading waveforms and the channel gains between the activated transmit antennas and the receiver antenna. The receiver signal can be expressed as follow: 


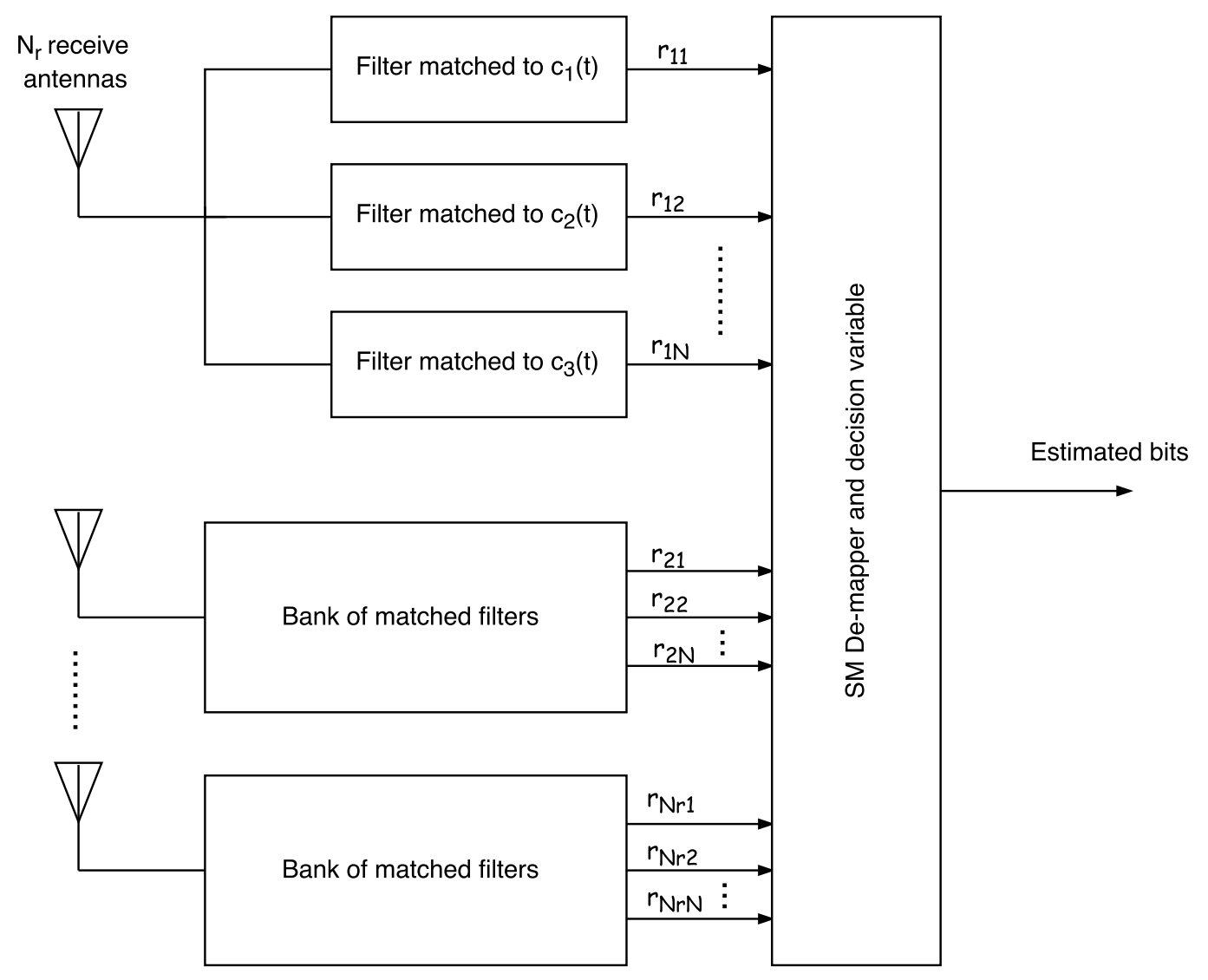

Figure 4.3: Receiver of MIMO/CDMA with permutation spreading using SM

$$
r_{x}^{(k)}=\sum_{i=1}^{N_{t}} \sqrt{E_{b}} b_{i}^{(k)} \alpha_{1 i}^{(k)} w_{i}^{(k)}+n^{(k)}
$$

with $E_{b}$ is the received energy per bit; $\alpha_{1 i}^{(k)}$ is the complex channel gain between the $i t h$ transmit antenna and the received antenna; $b_{i}^{(k)}$ is the message bits transmitted over the $i t h$ transmit antenna from the $k t h$ message vector; $w_{i}^{(k)}$ is the spreading sequence used by the $i t h$ transmit antenna and corresponding to $k$ th message vector. $n^{(k)}$ is the receiver noise when receiving the $k t h$ message.

As mentioned before in this section, only some of the transmit antennas will be active at each signaling interval. Thus $b_{i}^{(k)}$ in Formula 4.3 can either be equal to $\{0\}$ if the corresponding antenna is not used, or $\{-1\}$ or $\{1\}$, depending on the message bit to transmit, if the corresponding antenna is used. 
The output of the $l t h$ matched filter at the receive antenna when transmitting the $k t h$ message vector is:

$$
r_{l}^{(k)}= \begin{cases}\sum_{i=1}^{N_{t}} \sqrt{E_{b}} b_{i}^{(k)} \alpha_{1 i}^{(k)}+n_{l}^{(k)}, & \text { if } c_{l}^{(k)}(t) \text { is used } \\ n_{l}^{(k)}, & \text { otherwise }\end{cases}
$$

where $n_{l}^{(k)}$ is the sampled noise after the $l$ th matched filter when transmitting the $k t h$ message vector.

ML detection is then used in order to estimate transmit antenna index $(\hat{p})$ and data bits $(\hat{b})$ when the $k$ th message vector is transmitted; this is done by finding the spreading sequence that provides the minimum Euclidian distance between the received message vector and all possible message vectors. Therefore, the decision variable for MISO-CDMA system with permutation spreading sequences using SM is given as follows:

$$
(\hat{p}, \hat{b})=\min _{j=1}^{M}\left\|r_{x}^{(k)}-\sum_{i=1}^{N_{t}} \sqrt{E_{b}} b_{i}^{(k)} \alpha_{1 i}^{(k)} w_{i}^{(k)}\right\|^{2}
$$

where $\mathrm{M}$ is the total number of cosets.

Now let us consider a MIMO-CDMA system with permutation spreading sequences using SM with $N_{t}$ transmit antenna and $N_{r}$ receiver antenna. The received vector is expressed as follows:

$$
\boldsymbol{r}_{\boldsymbol{x}}=\left[r_{11}, r_{12}, \ldots, r_{1 N}, r_{21}, r_{22}, \ldots, r_{2 N}, \ldots, r_{N_{r} 1}, r_{N_{r} 2}, \ldots, r_{N_{r} N}\right]^{T}
$$

where $r_{j l}$ is the output from the $l t h$ matched filter of the $j t h$ receiver antenna and it is expressed as follows:

$$
r_{j l}^{(k)}= \begin{cases}\sum_{i=1}^{N_{t}} \sqrt{\frac{E_{b}}{N_{r}}} b_{i}^{(k)} \alpha_{i j}^{(k)}+n_{j l}^{(k)}, & \text { if } c_{l}^{(k)} \text { is used } \\ n_{j l}^{(k)}, & \text { otherwise }\end{cases}
$$


where $\alpha_{i j}^{(k)}$ is the complex channel gain between the $i t h$ transmit antenna and the $j t h$ receiver antenna when transmitting message $(k)$, and $n_{j l}^{(k)}$ is the sampled noise after the lth matched filter of the $j t h$ receiver antenna when message $(k)$ is transmitted.

After the output of the matched filter, the received vector would be given as:

$$
r_{x}=u_{b}+n
$$

where $\boldsymbol{n}$ is the $1 \mathrm{x} N \cdot N_{r}$ noise matrix: $\boldsymbol{n}=\left[n_{11}, \ldots, n_{1 N}, n_{21}, \ldots, n_{2 N}, \ldots, n_{N r 1}, . ., n_{N_{r} N}\right]^{T}$ and $\boldsymbol{u}_{b}$ is the $1 \mathrm{x} N . N_{r}$ received data matrix that depends on the transmitted data vector $\boldsymbol{b}=\left[b 1, b 2, \ldots, b_{N_{t}}\right]$.

For instance, if the message to be transmitted is $m=\left[\begin{array}{llll}1 & 0 & 1 & 1\end{array}\right]$, which is from coset $M_{5}$ according to Table 4.3. In this coset, antennas 2 and 4 are used and the last two bits of the message $m$ are to transmit. Then the data vector is $b=\left[\begin{array}{llll}0 & 1 & 0 & 1\end{array}\right]$ and $\boldsymbol{u}_{\boldsymbol{b}}=$ $\left[0,0,0,0,0, \alpha_{12}, 0, \alpha_{14}, 0,0,0,0,0, \alpha_{22}, 0, \alpha_{24}, \ldots, 0,0,0,0,0, \alpha_{N_{r} 2}, 0, \alpha_{N_{r} 4}\right]^{T}$.

Now, if the message to be transmitted is $m=\left[\begin{array}{llll}1 & 1 & 0 & 1\end{array}\right]$, which is from coset $M_{3}$ according to Table 4.3. In this coset, antennas 1 and 3 are used and the last two bits of the message $m$ are to transmit. Then the data vector is $b=\left[\begin{array}{llll}-1 & 0 & 1 & 0\end{array}\right]$ and $\boldsymbol{u}_{\boldsymbol{b}}=$ $\left[\alpha_{13}, 0,-\alpha_{11}, 0,0,0,0,0, \alpha_{23}, 0,-\alpha_{21}, 0,0,0,0,0, \ldots, \alpha_{N_{r} 3}, 0, \alpha_{N_{r} 1}, 0,0,0,0,0\right]^{T}$.

ML detection is then used in order to estimate transmit antenna index $(\hat{p})$ and data bits $(\hat{b})$ when the $k t h$ message vector is transmitted. Thus, the decision variable for MIMOCDMA system with permutation spreading sequences using SM is given by:

$$
(\hat{p}, \hat{b})=\stackrel{M}{\min _{j=1}}\left\|\boldsymbol{r}_{\boldsymbol{x}}-\hat{\boldsymbol{u}}_{\boldsymbol{b}}\right\|^{2}
$$

where $\hat{\boldsymbol{u}_{\boldsymbol{b}}}$ is the vector made up of all possible received vector with the absence of the noise. 


\subsection{Theoretical analysis of the BER performance}

In order to determine the analytical BER of the proposed system, the same method discussed in [28] and introduced in chapter 2 will be used under the same assumptions. Based on study shown in [28], the BER as well as the probability of symbol error, when using ML detection, can be computed by first finding the squared Euclidian distance between constellation points, then computing the union bound probability using formula $p(L, x)$ given by [29]:

$$
p(L, x)=\left(\frac{1-\mu}{2}\right)^{L} \quad \sum_{k=0}^{L-1}\left(\begin{array}{c}
L-1+k \\
k
\end{array}\right)\left(\frac{1+\mu}{2}\right)^{k}
$$

where

$$
\mu=\sqrt{\frac{x}{x+N_{r}}}
$$

$L$ is the diversity order, $x$ is average received energy per bit to noise spectral density ratio and $N_{r}$ is the number of receiver antenna.

\subsubsection{MISO-CDMA system}

Let us start with a MISO-CDMA system with permutation spreading sequences using SM with $N_{t}$ transmit antenna and one receiver antenna. The channels are assumed to be frequency-nonselective with independent gains. In this case, the received vector is given by:

$$
r_{\boldsymbol{x}}=\left[\boldsymbol{r}_{11}, \boldsymbol{r}_{12}, \ldots, \boldsymbol{r}_{1 N}\right]
$$

The first step of the analysis consists of finding the squared Euclidian distance between constellation points. For MISO system with four transmit antennas, the constellation points are shown in Table 4.4 where the noise is considered to be equal to zero. 


\begin{tabular}{|c|c|c|}
\hline Coset & Message $\mathbf{~ m}$ & Receiver vector $r_{x}$ \\
\hline \multirow{2}{*}{$M_{1}$} & 0000 & {$\left[\sqrt{E_{b}}\left(-\alpha_{11}, 0,-\alpha_{13}, 0,0,0,0,0\right)\right]$} \\
\cline { 2 - 3 } & 1111 & {$\left[\sqrt{E_{b}}\left(\alpha_{11}, 0, \alpha_{13}, 0,0,0,0,0\right)\right]$} \\
\hline \multirow{2}{*}{$M_{2}$} & 0001 & {$\left[\sqrt{E_{b}}\left(0,-\alpha_{11}, 0, \alpha_{13}, 0,0,0,0\right)\right]$} \\
\cline { 2 - 3 } & 1110 & {$\left[\sqrt{E_{b}}\left(0, \alpha_{11}, 0,-\alpha_{13}, 0,0,0,0\right)\right]$} \\
\hline \multirow{2}{*}{$M_{3}$} & 0010 & {$\left[\sqrt{E_{b}}\left(-\alpha_{13}, 0, \alpha_{11}, 0,0,0,0,0\right)\right]$} \\
\cline { 2 - 3 } & 1101 & {$\left[\sqrt{E_{b}}\left(\alpha_{13}, 0,-\alpha_{11}, 0,0,0,0,0\right)\right]$} \\
\hline \multirow{2}{*}{$M_{4}$} & 0011 & {$\left[\sqrt{E_{b}}\left(0, \alpha_{13}, 0, \alpha_{11}, 0,0,0,0\right)\right]$} \\
\cline { 2 - 3 } & 1100 & {$\left[\sqrt{E_{b}}\left(0,-\alpha_{13}, 0,-\alpha_{11}, 0,0,0,0\right)\right]$} \\
\hline \multirow{3}{*}{$M_{5}$} & 0100 & {$\left[\sqrt{E_{b}}\left(0,0,0,0,0,-\alpha_{12}, 0,-\alpha_{14}\right)\right]$} \\
\cline { 2 - 3 } & 1011 & {$\left[\sqrt{E_{b}}\left(0,0,0,0,0, \alpha_{12}, 0, \alpha_{14}\right)\right]$} \\
\hline \multirow{2}{*}{$M_{6}$} & 0101 & {$\left[\sqrt{E_{b}}\left(0,0,0,0,-\alpha_{12}, 0, \alpha_{14}, 0\right)\right]$} \\
\cline { 2 - 3 } & 1010 & {$\left[\sqrt{E_{b}}\left(0,0,0,0, \alpha_{12}, 0,-\alpha_{14}, 0\right)\right]$} \\
\hline \multirow{2}{*}{$M_{7}$} & 0110 & {$\left[\sqrt{E_{b}}\left(0,0,0,0,0,-\alpha_{14}, 0, \alpha_{12}\right)\right]$} \\
\cline { 2 - 3 } & 1001 & {$\left[\sqrt{E_{b}}\left(0,0,0,0,0, \alpha_{14}, 0,-\alpha_{12}\right)\right]$} \\
\hline \multirow{2}{*}{$M_{8}$} & 0111 & {$\left[\sqrt{E_{b}}\left(0,0,0,0, \alpha_{14}, 0, \alpha_{12}, 0\right)\right]$} \\
\cline { 2 - 3 } & 1000 & {$\left[\sqrt{E_{b}}\left(0,0,0,0,-\alpha_{14}, 0,-\alpha_{12}, 0\right)\right]$} \\
\hline
\end{tabular}

Table 4.4: Received vector for MISO system with four transmit antennas and one receiver antenna

\section{Probability of detecting message from the same coset}

Let us assume that the transmitted message is $m=\left[\begin{array}{llll}0 & 0 & 0 & 0\end{array}\right]$. To find the probability of detecting message $m=\left[\begin{array}{llll}1 & 1 & 1 & 1\end{array}\right]$, given that message $m=\left[\begin{array}{llll}0 & 0 & 0 & 0\end{array}\right]$ is transmitted, we should compute the squared Euclidean distance between their constellation points:

$$
\begin{aligned}
d_{15,0}^{2} & =\left\|r_{1111}-r_{0000}\right\|^{2} \\
& =\left|\sqrt{E_{b}}\left(\alpha_{11}-\left(-\alpha_{11}\right)\right)\right|^{2}+\left|\sqrt{E_{b}}\left(\alpha_{13}-\left(-\alpha_{13}\right)\right)\right|^{2} \\
& =4 E_{b}\left[\left|\alpha_{11}\right|^{2}+\left|\alpha_{13}\right|^{2}\right]
\end{aligned}
$$


Similar to study shown in [28], it can be shown that $d_{(15,0)}^{2}$ has a chi-square distribution with 4 degree of freedom and diversity order of 2 . Therefore, the probability that the message $m=\left[\begin{array}{llll}1 & 1 & 1 & 1\end{array}\right]$ is detected given that message $m=\left[\begin{array}{llll}0 & 0 & 0 & 0\end{array}\right]$ is transmitted is given by $[29]$ :

$$
p_{15}=p\left(2,4 \hat{\gamma}_{b}\right)
$$

where $\hat{\gamma_{b}}$ is the average received SNR per bit.

\section{Probability of detecting message from different coset but having the same antenna index}

In this section we investigate the probability that the receiver detects a different message that uses the same set of transmit antennas. From Table 4.4, it can be seen that three cosets $\left(M_{2}, M_{3}\right.$ and $\left.M_{4}\right)$ use the same transmit antenna combination. It can be shown that all message vectors from these cosets have the same Euclidean distance and therefore the same probability of error. We refer to this probability as $p_{\text {Same index }}$.

Let us use message $m=\left[\begin{array}{llll}1 & 1 & 0 & 0\end{array}\right]$ from coset $M_{4}$ as an example. The distance between the constellation points of this message and message $m=\left[\begin{array}{llll}0 & 0 & 0 & 0\end{array}\right]$ is shown as follows:

$$
\begin{aligned}
d_{12,0}^{2} & =\left\|r_{1100}-r_{0000}\right\|^{2} \\
& =\left|\sqrt{E_{b}} \alpha_{11}\right|^{2}+\left|\sqrt{E_{b}} \alpha_{11}\right|^{2}+\left|\sqrt{E_{b}} \alpha_{13}\right|^{2}+\left|\sqrt{E_{b}} \alpha_{13}\right|^{2} \\
& =2 E_{b}\left[\left|\alpha_{11}\right|^{2}+\left|\alpha_{13}\right|^{2}\right]
\end{aligned}
$$

It can be shown that $d_{12,0}^{2}$ has a chi-square distribution with 4 degree of freedom and

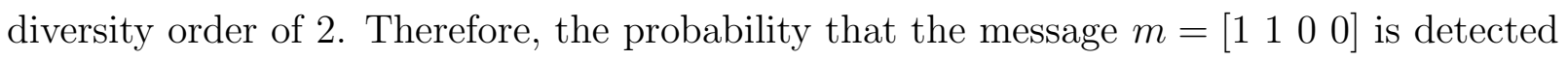
given that message $m=\left[\begin{array}{llll}0 & 0 & 0 & 0\end{array}\right]$ is transmitted is given as follows:

$$
p_{\text {Same index }}=p\left(2,2 \hat{\gamma_{b}}\right)
$$




\section{Probability of detecting message from different coset with different antenna index}

The last case of this analysis is when the receiver incorrectly detects a message from a different coset that uses a different transmit antenna combination than the transmitted message. From Table 4.4, it can be seen that the messages of four cosets $\left(M_{5}, M_{6}, M_{7}\right.$ and $\left.M_{8}\right)$ use a different transmit antenna combination than message $m=\left[\begin{array}{llll}0 & 0 & 0 & 0\end{array}\right]$. It can be shown that all message vectors from these cosets have the same Euclidean distance and therefore the same probability of error. We refer to this probability as $p_{\text {Diff }}$ index .

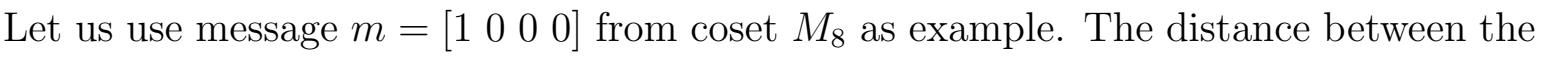
constellation points of this message and message $m=\left[\begin{array}{llll}0 & 0 & 0 & 0\end{array}\right]$ is shown as follows:

$$
\begin{aligned}
d_{8,0}^{2} & =|| r_{1000}-r_{0000} \|^{2} \\
& =\left|\sqrt{E_{b}} \alpha_{11}\right|^{2}+\left|\sqrt{E_{b}} \alpha_{13}\right|^{2}+\left|\sqrt{E_{b}} \alpha_{12}\right|^{2}+\left|\sqrt{E_{b}} \alpha_{14}\right|^{2} \\
& =E_{b}\left[\left|\alpha_{11}\right|^{2}+\left|\alpha_{12}\right|^{2}+\left|\alpha_{13}\right|^{2}++\left|\alpha_{14}\right|^{2}\right]
\end{aligned}
$$

It can be shown that $d_{12,0}^{2}$ has a chi-square distribution with 8 degree of freedom and

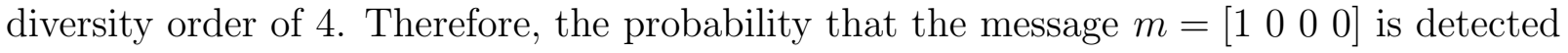
given that message $m=\left[\begin{array}{llll}0 & 0 & 0 & 0\end{array}\right]$ is transmitted is given as follows:

$$
p_{\text {Diff index }}=p\left(4, \hat{\gamma}_{b}\right)
$$

To estimate the probability of error detection when message $m=\left[\begin{array}{llll}0 & 0 & 0 & 0\end{array}\right]$ is transmitted, the union bound can be used [29]:

$$
\begin{aligned}
& P\left(E \mid\left[\begin{array}{llll}
0 & 0 & 0 & 0
\end{array}\right]<\sum_{i=1}^{15} p_{i \mid 0}\right. \\
& <p_{15}+6 * p_{\text {Same index }}+8 * p_{\text {Diff index }} \\
& <p\left(2,4 \hat{\gamma_{b}}\right)+6 * p\left(2,2 \hat{\gamma_{b}}\right)+8 * p\left(4, \hat{\gamma_{b}}\right)
\end{aligned}
$$


As the probability of error detection is independent of the transmitted message [28], we can write that $P(E)=P(E \mid[0000])$. Likewise, the probability of bit error is independent of the message that has been sent; the union bound probability can be expressed as follows:

$$
p_{b}<p\left(2,4 \hat{\gamma_{b}}\right)+3 * p\left(2,2 \hat{\gamma_{b}}\right)+4 * p\left(4, \hat{\gamma_{b}}\right)
$$

with $p(L, x)$ is computed using Equation 4.10.

\subsubsection{MIMO-CDMA system}

Now let us consider MIMO-CDMA system with permutation spreading sequences using SM with $N_{t}$ transmit antennas and $N_{r}$ receiver antennas. Under the same assumptions as section 4.3.1, the received vector is given by:

$$
\boldsymbol{r}_{\boldsymbol{x}}=\left[\boldsymbol{r}_{11}, \boldsymbol{r}_{12}, \ldots, \boldsymbol{r}_{1 N}, \boldsymbol{r}_{21}, \boldsymbol{r}_{22}, \ldots, \boldsymbol{r}_{2 N}, \ldots, \boldsymbol{r}_{N_{r} 1}, \boldsymbol{r}_{N_{r} 2}, \ldots, \boldsymbol{r}_{N_{r} N}\right]
$$

For instance, if the transmitted message is $m=\left[\begin{array}{llll}0 & 0 & 0 & 0\end{array}\right]$, the received vector is shown as:

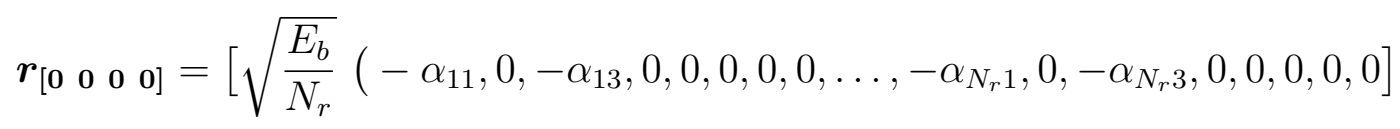

Following the same steps as the previous section, the probability of detecting message

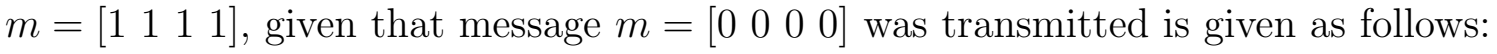

$$
p_{15}=p\left(2 N_{r}, 4 \hat{\gamma_{b}}\right)
$$

Similarly, the probability of detecting messages coming from different cosets but having the same transmit antenna index is:

$$
p_{\text {Same index }}=p\left(2 N_{r}, 2 \hat{\gamma}_{b}\right)
$$


Finally, the probability of detecting messages coming from different cosets and having different transmit antenna combination is:

$$
p_{\text {Diff index }}=p\left(4 N_{r}, \hat{\gamma}_{b}\right)
$$

Therefor, the union bound probability of a single user MIMO-CDMA system with permutation spreading sequences using SM is given by:

$$
p_{b}<p\left(2 N_{r}, 4 \hat{\gamma_{b}}\right)+3 * p\left(2 N_{r}, 2 \hat{\gamma_{b}}\right)+4 * p\left(4 N_{r}, \hat{\gamma_{b}}\right)
$$

with $p(L, x)$ is computed using Equation 4.10.

\subsection{Simulation results and discussion}

The BER performance of the proposed technique is discussed in this section where MIMOCDMA systems with two or four transmit antennas and one, two or four receiver antennas are considered. Simulations are performed under the same assumptions as chapter 2 and 3. All channel gains are considered to be independent, frequency non-selective and perfect channel state information (CSI) is considered to be available at the receiver with zero power penalties. The orthogonal spreading codes are generated using Hadamard codes with length of 8 bits per chip sequence.

\subsubsection{Theoretical and Simulation results of MIMO/CDMA with SM design}

Figure 4.4 and 4.5 show a comparison between the theoretical union bound of MISO and MIMO-CDMA systems with permutation spreading using conventional STBC design vs SM design. In Figure 4.4, MISO-CDMA system with four transmit antennas and one receiver antenna is considered. Equation 4.20 and 2.20 with $N_{r}=1$ are used. It can be seen that the new technique shows a significant improvement of the BER performance of 
about $2 \mathrm{~dB}$ for an SNR between 0 and $4 \mathrm{~dB}$. This is due mainly to the fact of using less transmit antennas and therefore reducing the inter-channel interferences. However, using fewer antennas means less diversity gain. This can be noticed from Figure 4.4 where the BER performance starts to drop for an SNR higher than 6dB.

In Figure 4.5 a MIMO-CDMA system with four transmit antennas and four receiver antennas is considered. Formulas 4.26 and 2.20 with $N_{r}=4$ are used. Same remarks as Figure 4.5 can be observed, the performance is significantly improved for an SNR between 0 and $10 \mathrm{~dB}$. Since this system uses more transmit antennas, we can notice the additional diversity gain effect where the BER performance starts to drop after an SNR of $12 \mathrm{~dB}$ compared to $8 \mathrm{~dB}$ for the MISO system shown in Figure 4.5.

Figure 4.6 presents the simulation vs, theoretical results of the BER performance of the proposed technique in MIMO-CDMA systems with 4 and 2 transmit antenna and 1, 2 and 4 receiver antennas. As expected, we can notice that the union bound estimation derived in the previous section is close to the simulation results and it is tight as the SNR increases. This is due because the union bound estimation doesn't include the probability of intersection events which tends towards zero as the SNR increases. We also notice additional diversity gain as the number of antennas increases which improves the BER performances.

\subsubsection{Comparison of simulation results}

In this section we compare the simulated results of the proposed technique and the conventional STBC design and the conventional SM design.

Figure 4.7, 4.8 and 4.9 show, respectively, the simulated BER performances of the different techniques for 2 and 4 transmit antennas with 1, 2 and 4 receive antennas. It can be seen that the new SM design provides a significant improvement of the BER performance, of about $2 \mathrm{~dB}$ for $4 \mathrm{x} 4 \mathrm{MIMO}$ system, compared to the conventional STBC technique. Also, we can notice that the BER performance start to drop as the SNR increases. However, an important increase of the BER performance can be noticed as the number of antennas increases. 


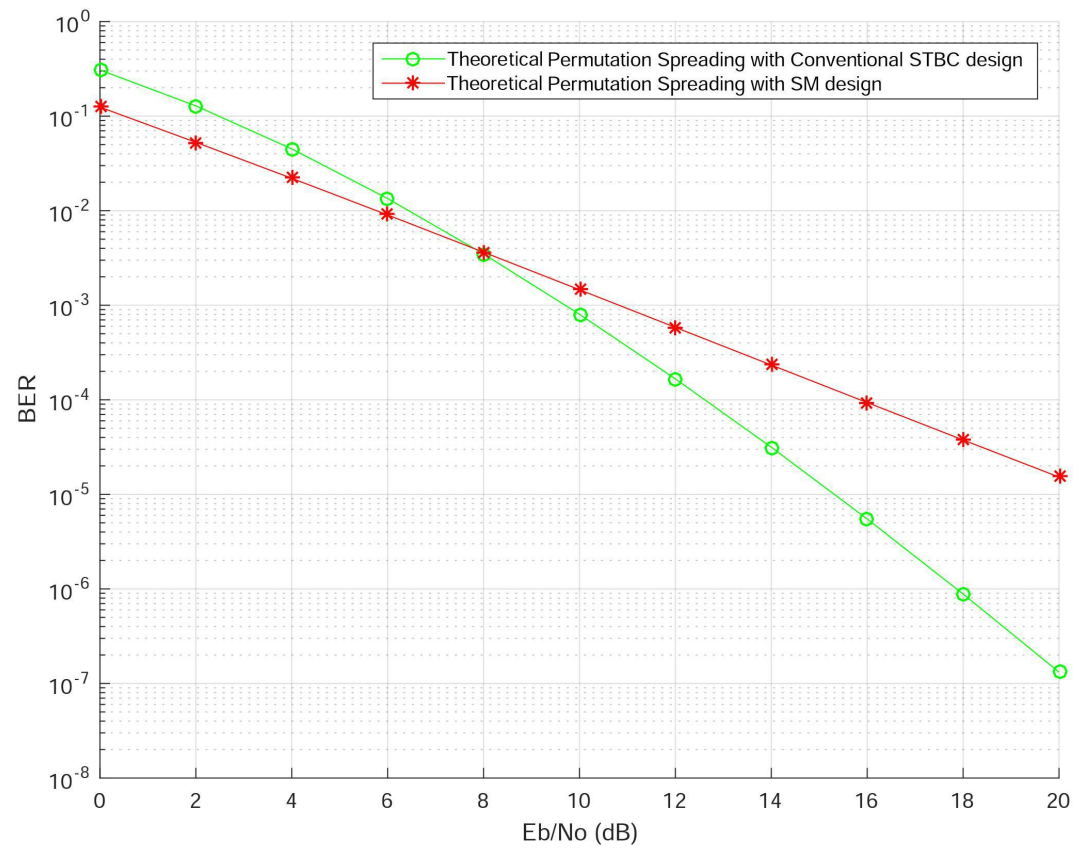

Figure 4.4: Theoretical BER performance of 4x1 MISO-CDMA System using Permutation Spreading with conventional STBC design vs SM design

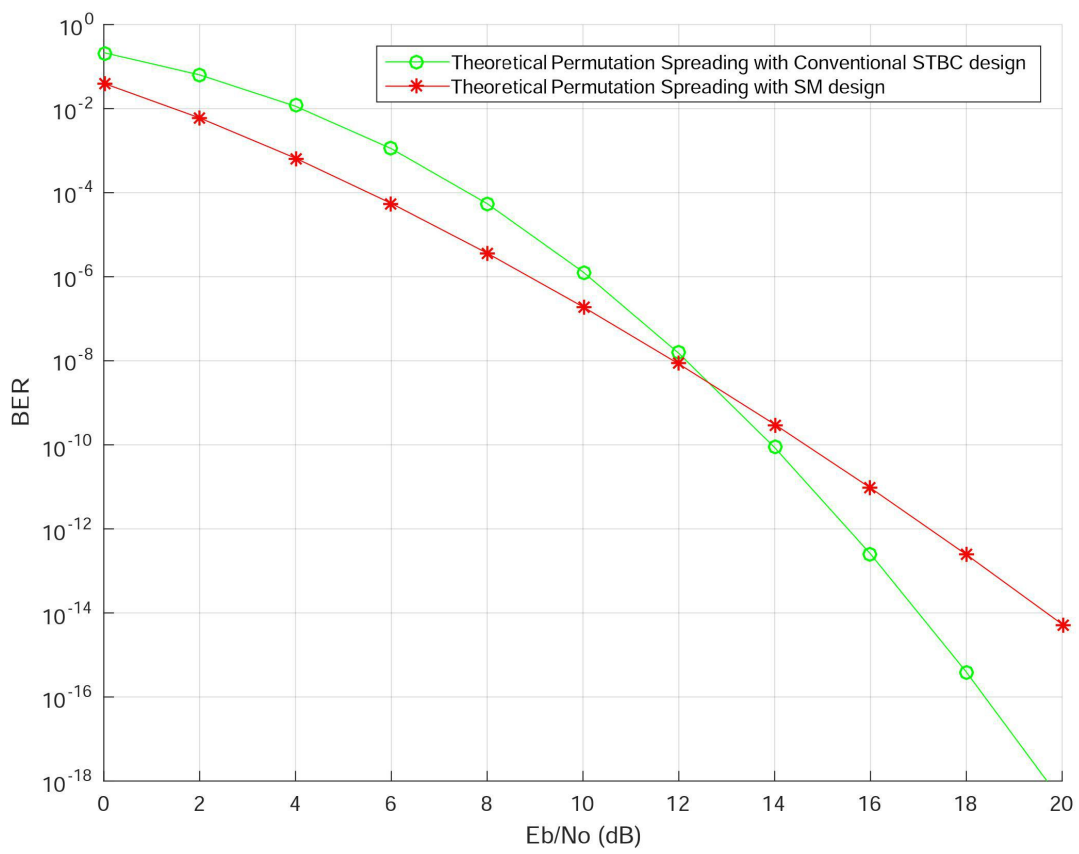

Figure 4.5: Theoretical BER performance of 4x4 MIMO-CDMA System using Permutation Spreading with conventional STBC design vs SM design 


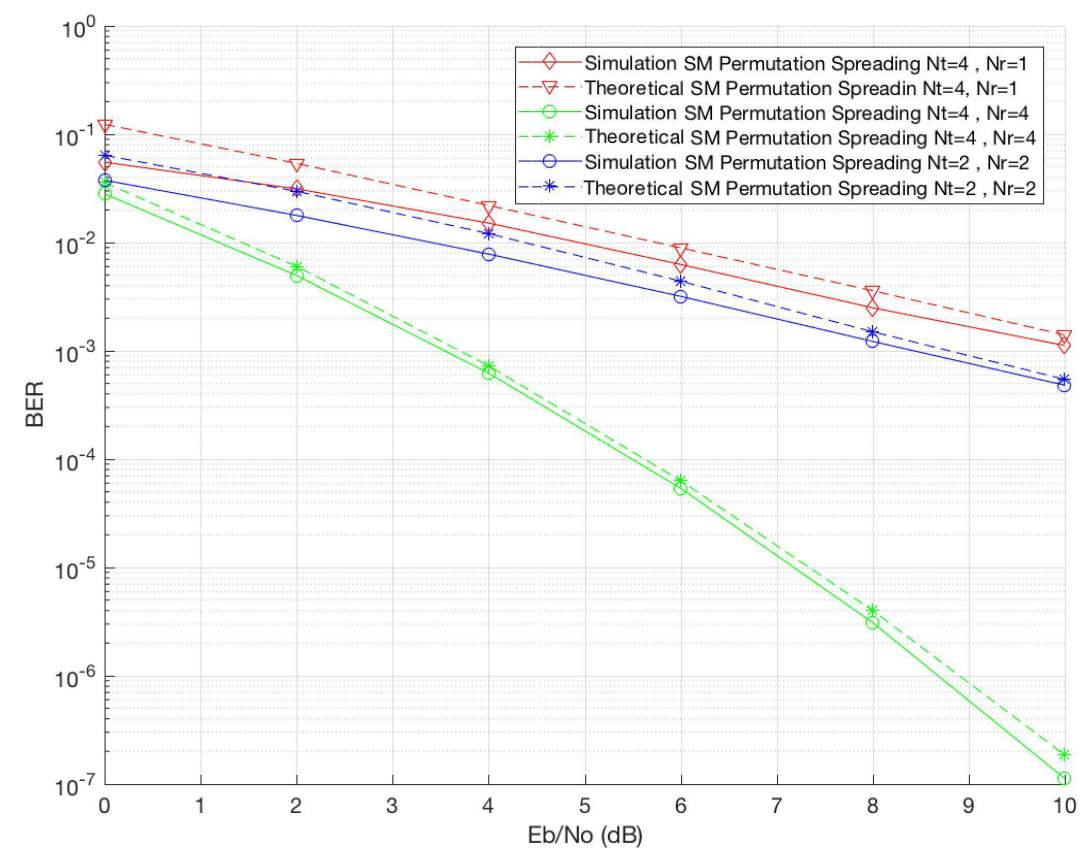

Figure 4.6: BER performance of MIMO-CDMA System with Permutation Spreading using SM design

Figure 4.9 shows simulation performance of MIMO systems with spectral efficiency of $\eta=4 b i t s / s / H z$ using the conventional SM techniques discussed in Chapter 2 and the new SM permutation spreading proposed in this chapter. The number of transmit antennas are set in order to achieve this spectral efficiency. Thus, For the conventional SM techniques $N_{r}=4$ and $N_{t}=8$ for SM, $N_{t}=5$ and $N_{a}=2$ for GSM and $N_{t}=4$ for VGSM; where for the proposed technique $N_{t}=4$ and $N_{r}=4$. We have to note that the new design uses 8 bits, for the spreading code, to transmit one bit at each time instant, whereas in SM only one bit is transmitted at each signaling. However, as it will be shown in Chapter 5, the simulation results show that when CDMA with SM permutation spreading is used a slightly significant BER performance gain is achieved. This improvement is achieved because the used design creates dependency between parallel bits and spreading waveforms in addition to the dependency between bits and antenna combinations. 


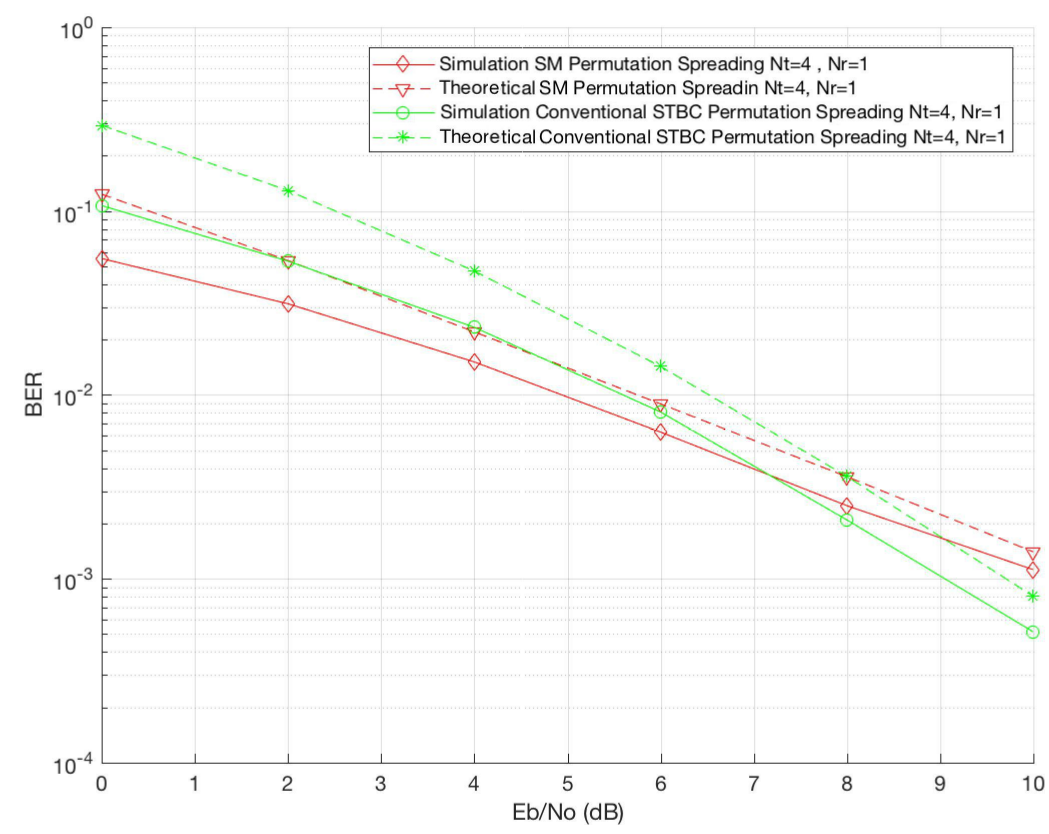

Figure 4.7: BER performance of 4x1 MISO-CDMA System using Permutation Spreading with conventional STBC design vs SM design

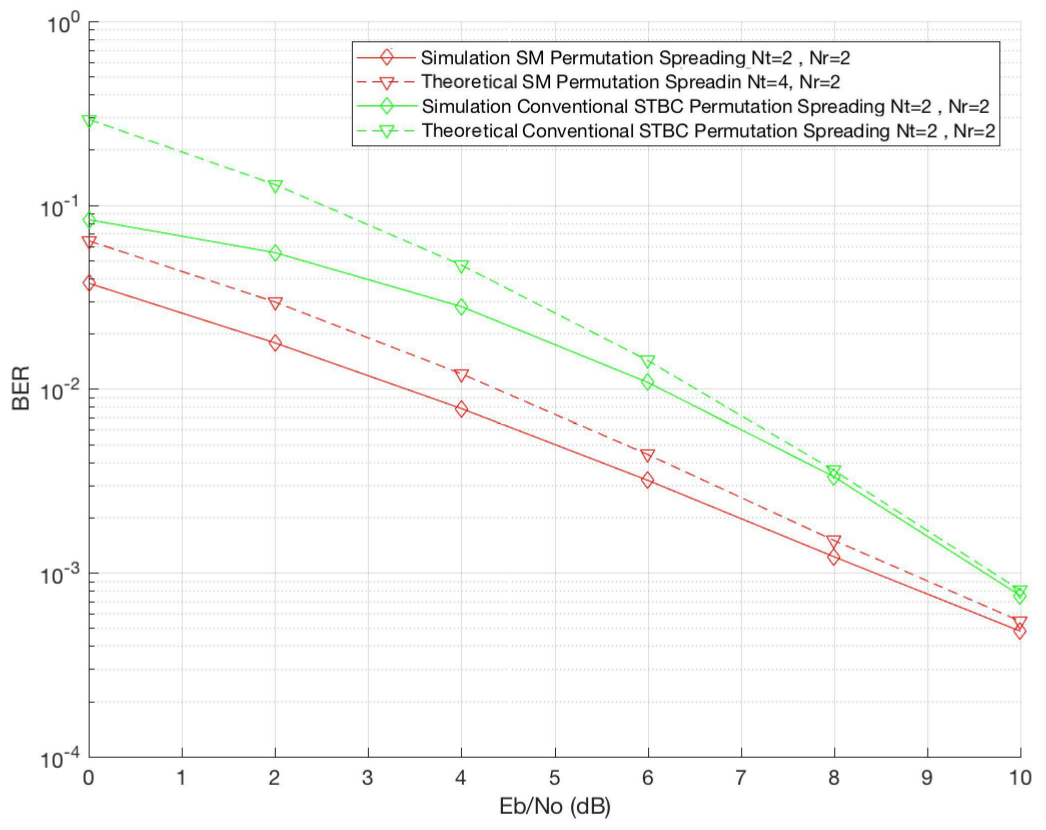

Figure 4.8: BER performance of 2x2 MIMO-CDMA System using Permutation Spreading with conventional STBC design vs SM design 


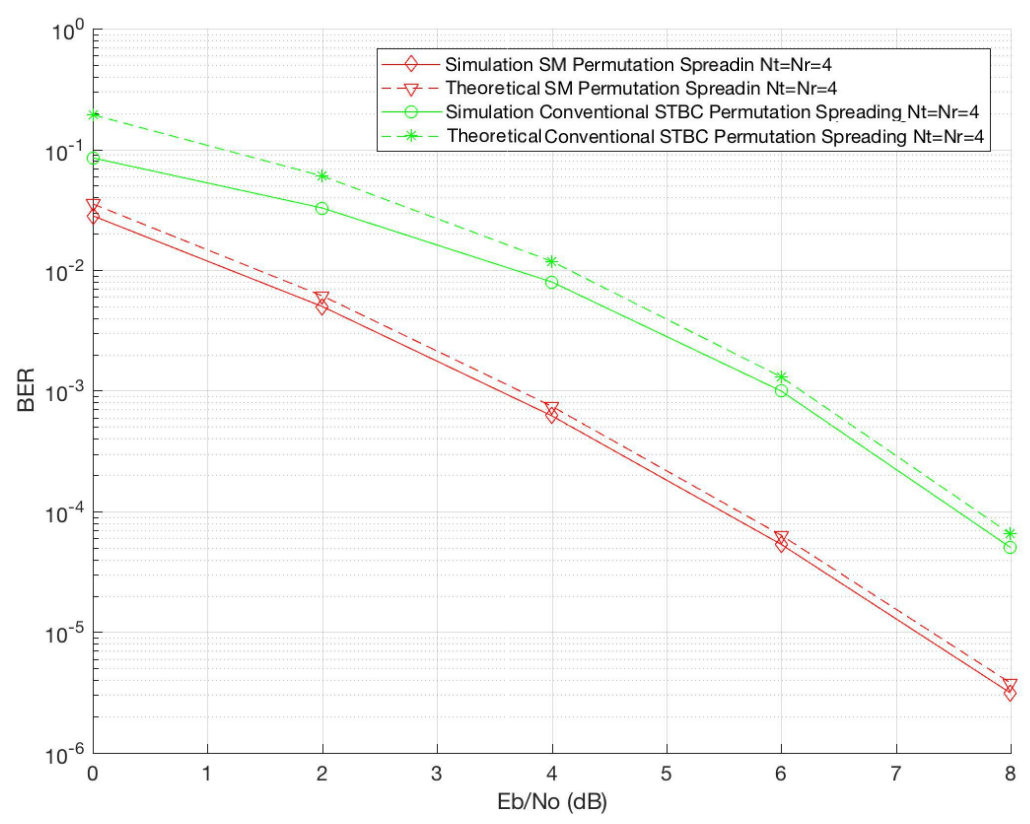

Figure 4.9: BER performance of 4x4 MIMO-CDMA System using Permutation Spreading with conventional STBC design vs SM design

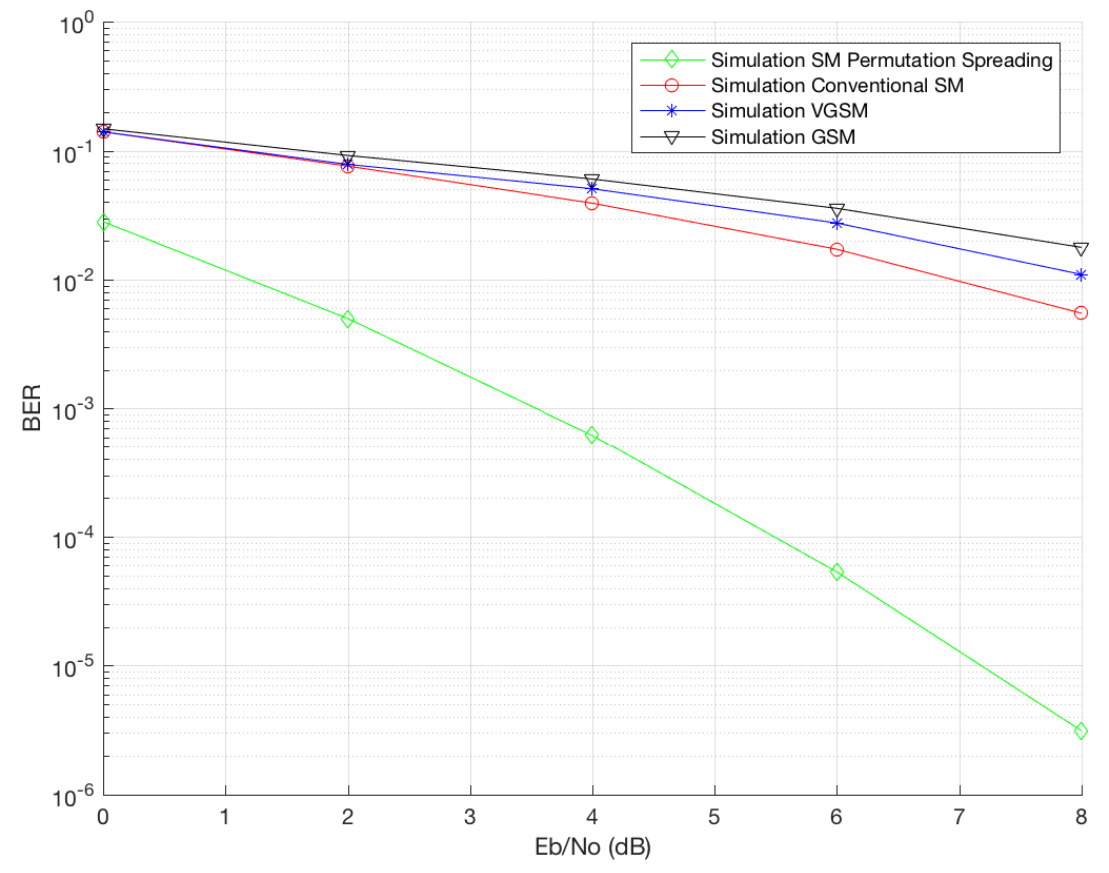

Figure 4.10: BER performance of MIMO-CDMA System using Permutation Spreading with SM design vs Conventional SM techniques 


\subsubsection{Discussion}

In this chapter, a novel technique for MIMO-CDMA system with permutation spreading using SM is introduced. It is shown how the spatial positions of transmit antennas can be combined with permutation spreading technique in order to create an additional dependency between the data bits and the antennas combinations. This leads to an enhance of the system performance of MIMO systems. An analytical expression of the BER was derived for the proposed technique under a frequency non-selective Rayleigh fading channel with independent gains and perfect knowledge of the channel state information (CSI) at the receiver. The obtained results were compared to the conventional permutation spreading technique using STBC design. Results show that the proposed technique provides significant improvement of the BER performance of about 2dB for an SNR less than $10 \mathrm{~dB}$ in the case of a $4 \times 4$ MIMO system compared to STBC design. Moreover, a comparison between the analytical expression and the simulated performance was shown. The comparison shows that the theoretical expression is close to the simulation results and it is tight as the SNR increases. Finally, a comparison between the proposed technique and the conventional STBC and SM design was presented. Simulation results show that the new SM designs outperform the performance of the conventional techniques. 


\section{Chapter 5}

\section{Multiuser Permutation Spreading Employing Spatial Modulation For Synchronous MIMO-CDMA systems}

\subsection{Introduction}

In the MIMO-CDMA systems that were discussed in the previous chapter, only the single user scenarios were treated for the proposed permutation spreading technique. In this chapter, the focus shifts to multiple user scenario in synchronous transmission. In such a model, each user generates exactly one message vector during the signalling interval and all users signals are received by the receiver with no relative delay. Therefore, at the receiver, all users' messages begin and end at the same time. This is unlike the asynchronous case, where the interference caused to one user's message has contributions from two of each of the other users' messages [29]. This multiple access interference (MAI) hugely affects the BER performance of all users who transmit simultaneously on the channel, and thus, an investigation of the effect of MAI on BER of our proposed system is needed..

In CDMA systems, all users share the same spectrum by using a set of mutually orthogonal spreading sequences that are unique for each user [40]. However, this requires increasing the length of the spreading sequences (chip rate) and thus reducing the spectral 
efficiency. Another solution can be envisaged in order to improve the spectral efficiency is by creating spreading codes with low cross-correlation properties, which leads to interference between different users. These codes are called pseudo-noise (PN) sequences. Each is assigned a unique PN that is multiplied by the spreading sequences before transmission. In this chapter, the receiver is assumed to have perfect knowledge of the used signatures per user.

In CDMA multiusers systems, the received signal of users close to the base station (BS) will have higher SNR than those far from the BS, which in turn cause higher interference to other users than the far users do. This is known as the near-far effect [41]. In order to avoid this issue, power control techniques are used to mitigate the large scale fading by adjusting the transmit power of users so that their received powers are nearly the same. We assume that power control perfectly matches each users' average signal strength but is not fast enough to mitigate the small scale fading experienced by each user's transmission $[41]$.

Simulation results presented in this chapter show that the performances of the proposed technique overcome the traditional designs.

\subsection{System Model}

\subsubsection{Transmitter Model}

The transmitter block diagram of a multiuser MIMO-CDMA system using the permutation spreading technique with SM design is shown in Figure 5.1. We consider an uplink channel of a MIMO-CDMA system with $Q$ users in which each user is equipped with $N_{t}$ transmit antennas and the receiver (BS) is equipped with $\mathrm{Nr}$ receiver antennas. As previously explained, all users have access to the same spectrum and are separated by assigning a unique signature waveform to each user.

Similar to the single user system discussed in the chapter before, the data bit streams $\left\{d_{q}[i]\right\}$ for user $q=1,2, \ldots, Q$, are first divided into blocks of $k$ bits, where $k=\log _{2}(M) *$ 
$N_{t}$ ) for an M-ary modulation scheme. The message bits are then converted into $N_{t}$ parallel streams and fed into the mapping blocks. Based on the input bits, the spreading code selector and the SM-mapping block choose the pair of antenna to activate and a unique spreading sequences $\left(w_{1}^{q}(t), w_{2}^{q}(t), \ldots, w_{N_{t}}^{q}(t)\right)$ from $N$ set of mutually orthogonal sequences $\left(c_{1}(t), c_{2}(t), \ldots, c_{N}(t)\right)$. The spreading sequences are used to spread the BPSK modulated bits to transmit. The last step of the transmitting process is the scrambling operation, which is done by multiplying the final symbols to the pseudo-noise $(\mathrm{PN})$ sequence.

The scrambling that can be used should have low cross-correlation values in order to reduce the multiple-access interference and increase the number of users that can share the same spectrum. Two codes are well known to meet this condition, Kasami codes and Gold codes [42]. In this thesis, Gold codes are used to generate the pseudo-noise (PN) sequences of a length of 8 bits per chips. The codes are chosen such a way to have a cross-correlation near to zero.

\subsubsection{Receiver Model}

The receiver block diagram of a multiuser MIMO-CDMA system using permutation spreading technique with SM design is depicted in Figure 5.2. As all users use the same spreading sequences, the receiver will be equipped with a bank of matched filters that matches one of the $N$ spreading waveforms $\left(c_{1}(t), c_{2}(t), \ldots, c_{N}(t)\right)$. The receiver first multiplies the received signal by the pseudo-noise $(\mathrm{PN})$ code of the desired user and then treats all the other users' signals as additional noise. The receiver uses ML to estimate the transmit antennas index and the transmitted symbol of the desired user by comparing the received vector with all possible transmitted combinations and selects the option that provides the minimum Euclidian distance.

\section{Decision variable}

Lets consider an uplink MIMO-CDMA system employing SM permutation spreading sequences with $Q$ users. Each user is equipped with $N_{t}$ transmit antennas and the base station (BS) is equipped with $N_{r}$ receiver antennas. For such a system, the decision 

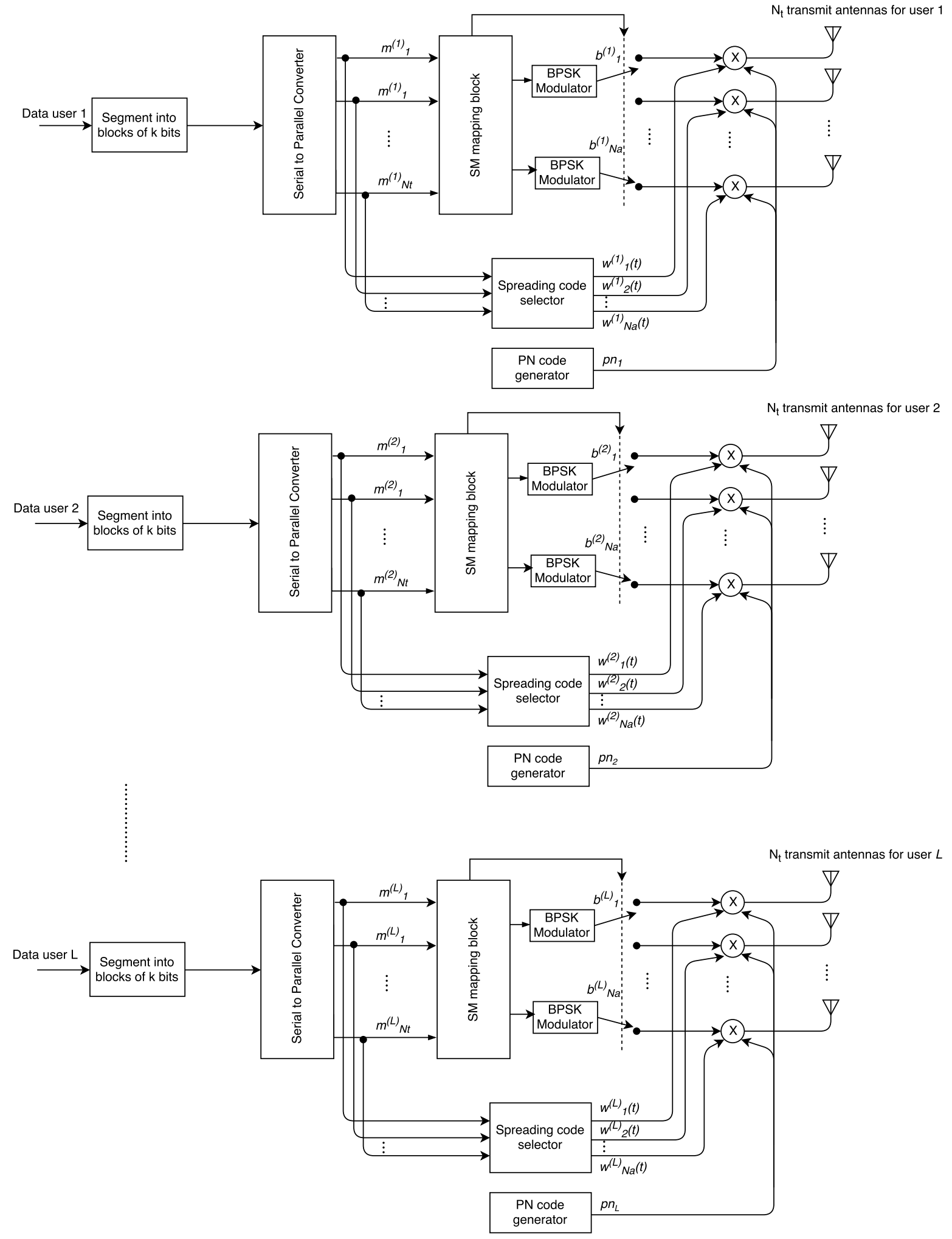

Figure 5.1: Multiuser transmitter of MIMO-CDMA with permutation spreading using SM 


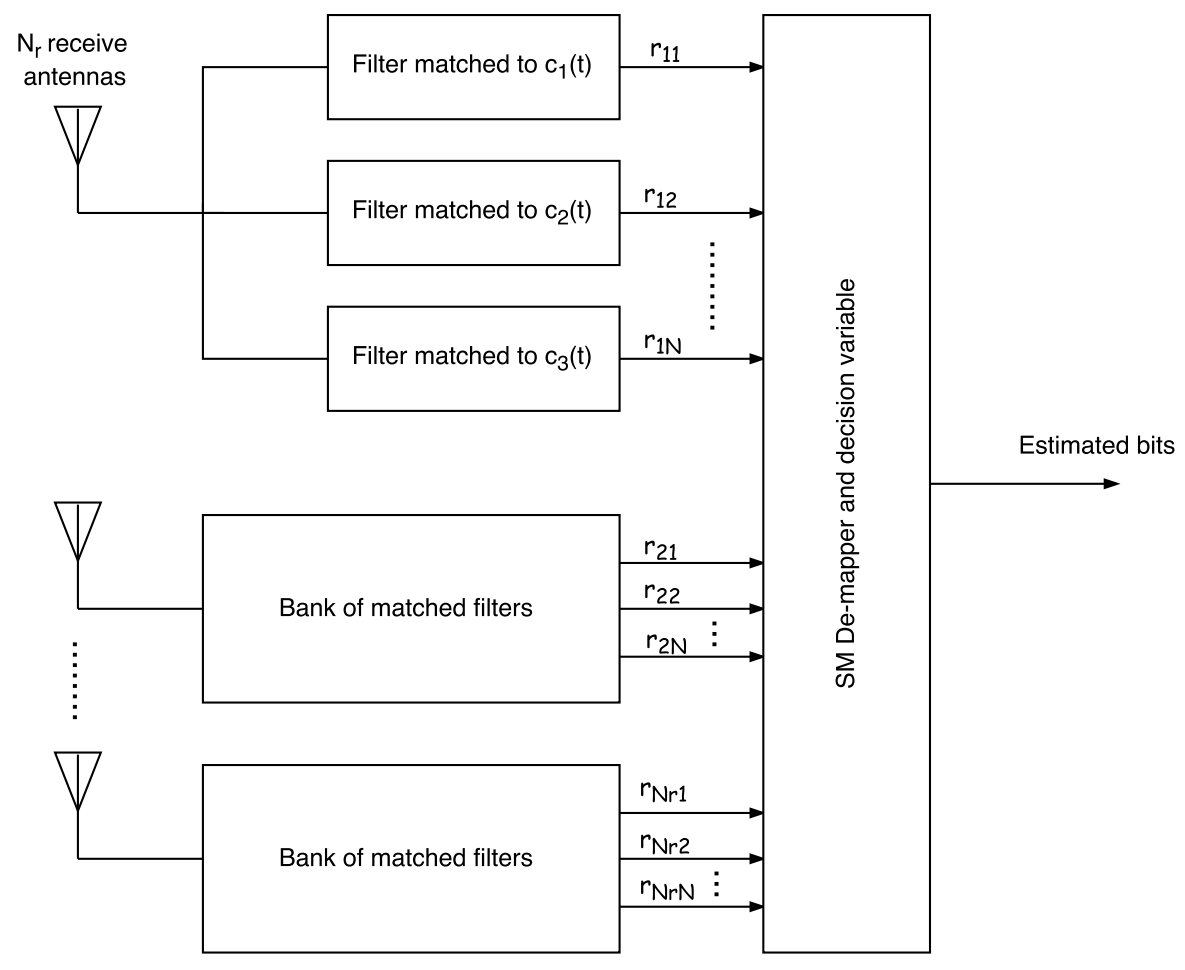

Figure 5.2: Multiuser receiver of MIMO/CDMA with permutation spreading using SM variable could be derived the same way in the single user scenario discussed in Chapter 4. The received vector is given by:

$$
\boldsymbol{r}_{\boldsymbol{x}}=\left[r_{11}, r_{12}, \ldots, r_{1 N}, r_{21}, r_{22}, \ldots, r_{2 N}, \ldots, r_{N_{r} 1}, r_{N_{r} 2}, \ldots, r_{N_{r} N}\right]^{T}
$$

where $r_{j l}$ is the output from the $l t h$ matched filter of the $j t h$ receiver antenna and it is expressed as follows:

$$
r_{j l}=\sum_{q=1}^{Q} \sum_{i=1}^{N_{t}} \sqrt{\frac{E_{b}}{N_{r}}} b_{i}^{q} \alpha_{i j}^{q} w_{i}^{q} p_{n}^{q}+n_{j l}
$$

The notation used in 5.2 is given as follows:

- $\alpha_{i j}^{q}$ is the complex channel gain between the $i t h$ transmit antenna and the $j t h$ receiver antenna for the qth user. 
- $n_{j l}$ is the sampled noise after the $l t h$ matched filter of the $j t h$ receiver antenna.

- $b_{i}^{q}$ is bit symbol transmitted by the qth user using antenna $i$.

- $p_{n}^{q}$ is the pseudo-noise code generated for the $q$ th user.

For a given user $q$, the receiver treats the other users' signals as noise and uses ML to estimate the transmitted data of the qth user. In fact, if we consider that the desired signal to process is from user 1 , the received signal could be rewritten as follows:

$$
r_{x}=u_{b}^{1}+n^{\prime}
$$

where:

- $\boldsymbol{n}^{\prime}$ is the noise matrix and it's given by: $\boldsymbol{n}^{\prime}=\left[n_{11}^{\prime}, \ldots, n_{1 N}^{\prime}, n_{21}^{\prime}, \ldots, n_{2 N}^{\prime}, n_{N_{r} 1}^{\prime}, \ldots, n_{N r N}^{\prime}\right]$ where $n_{j l}^{\prime}=\sum_{q=2}^{Q} \sum_{i=1}^{N_{t}} \sqrt{\frac{E_{b}}{N_{r}}} b_{i}^{q} \alpha_{i j}^{q} w_{i}^{q}(t) p_{n}^{q}+n_{j l}$

- $u_{b}=\sum_{i=1}^{N_{t}} \sqrt{\frac{E_{b}}{N_{r}}} b_{i}^{1} \alpha_{i j}^{1} w_{i}^{1}(t) p_{n}^{1}$ is the received data matrix from the 1st receiver.

The decision variable, of the $q$ th user, for a multiuser MIMO-CDMA system with permutation spreading sequences using spatial modulation is given by:

$$
(\tilde{p}, \tilde{b})=\min _{j=1}\left\|\boldsymbol{r}_{x}-\tilde{\boldsymbol{u}}_{\boldsymbol{b}}^{q}\right\|^{2}
$$

where $\tilde{\boldsymbol{u}}_{\boldsymbol{b}}^{q}$ is the vector made up of all possible received vector, of the desired user $q$ with the absence of the noise.

\subsection{Simulation results and discussion}

\subsubsection{Simulation results comparison}

The simulation results of the BER performance are shown in this section. We consider a synchronous MIMO-CDMA system with 4 transmit antennas and 4 receiver antennas. 
The simulations are performed under the same assumptions as chapter 4 where all channel gains are considered to be independent, frequency non-selective and perfect channel state information (CSI) is considered to be available at the receiver with zero power penalties. We assume that all users are assigned with the same orthogonal spreading codes. These sequences are generated using Hadamard codes with length of 8 bits per chip sequence. The selection of the spreading sequences and the transmit antennas to activate are based on Table 4.3. Pseudo-noise (PN) sequences are used to separate each user and they are generated using Gold codes with a length of 8 bits per chip. The codes are chosen such a way to minimize the cross-correlation value [43]. Finally, we assume that all users have the same averaging SNR.

The BER performance of the multiuser MIMO-CDMA system employing permutation spreading sequences with SM design for 2, 3 and 4 users scenario are shown in Figure 5.3. A comparison between all these scenarios and the STBC permutation spreading sequences for a multiuser MIMO-CDMA system with 4 transmit antennas and 4 receiver antennas is shown in Figure 5.4.

From these figures, it can be seen that the BER performance decreases as more as we add users to the system. Moreover, we can observe that at high SNR values an error floor for a system with 3 users start to appear for a BER around $3 * 10^{-5}$ and around $10^{-4}$ for 4 users system. In the single user system discussed in chapter 4, only a pair of transmit antenna is activated at each signalling interval, which decreases the ICI and therefore enhance the system performance. However, in the multiuser system more transmit antennas are involved which increase the ICI effect and reduce the BER performance as it can be observed from the simulation results.

In order to keep the same spectral efficiency, all users use the same orthogonal spreading sequences and scrambling codes are used to separate users from each other. However, these codes are not perfectly orthogonal and their cross-correlations are not always near to zero. Thus, for high SNR we can observe that more we increase the number of users more the probability of having sequences with high cross-correlation values increases. This effect leads to situations where codes sequences with low minimum distance are used, which 
results the error floor.

The comparison between the SM design and the STBC design presented in Figure 5.4. shows that the new design outperform the conventional method. It can be seen that the 4 users system with SM design provides better performance than the single user system with STBC design for a BER greater than $6 * 10^{-4}$.

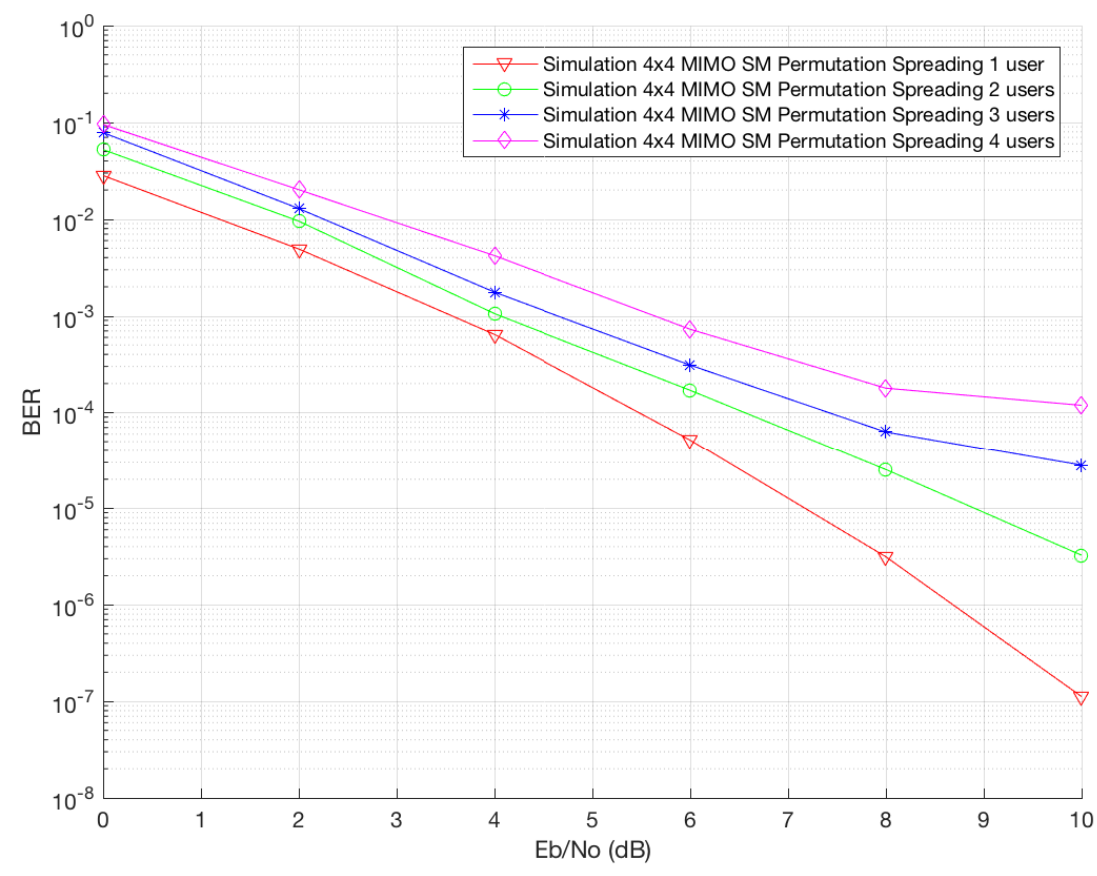

Figure 5.3: Comparison of BER Performance of 4x4 MISO-CDMA System using SM Permutation Spreading design

\subsubsection{Discussion}

In this chapter, the multiusers scenario for the proposed MIMO-CDMA system with permutation spreading sequences using SM is discussed. In such a system, all users are considered to share the same spreading sequences and use scrambling codes to separate user's information from each other. The receiver estimates the desired user information by considering the other users' signal as additional noise. The simulation results show that the new design still outperforms the STBC design in synchronous multiuser transmission. However, for high SNR values the SM design has higher error floor compared to the STBC 


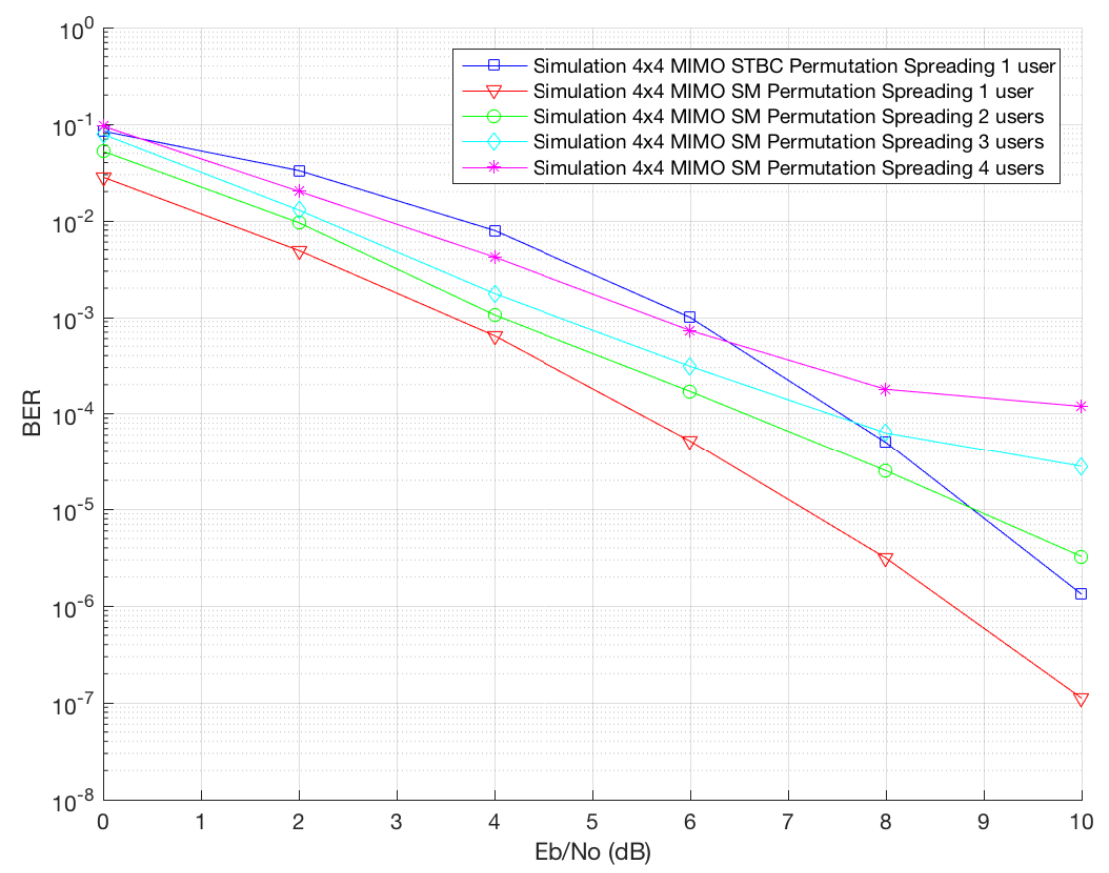

Figure 5.4: Comparison of BER performance of 4x4 MISO-CDMA System using SM Permutation Spreading design vs STBC design

design. This is because the cross-correlation characteristics of the scrambling codes start to drop as the number of users increases. Furthermore, the low diversity order of the SM design could affect the performance of the system at high SNR values. In order to decrease the effects of the MAI, several methods could be used such as increasing the length of the signature codes in order to enhance the cross-correlation characteristics. Also, multiuser detection (MUD) methods are a good candidate to increase the system performance by estimating the MAI of all users and then subtracted out from the received signal. 


\section{Chapter 6}

\section{Conclusion and suggestion for future wOrk}

\subsection{Conclusion}

In this thesis, we have proposed a new modulation technique for MIMO-CDMA systems that takes the advantage of both SM-design and permutation spreading design. This study discussed the benefits of combining the conventional permutation spreading techniques for MIMO-CDMA with the new SM design and how could this strategy increase the system performance in terms of spectral and power efficiency for single and multiuser scenarios.

In chapter 2, the concept of the parity bit selected sequences and permutation spreading sequences for MIMO-CDMA systems was presented. The chapter discussed the difference between the existing design strategies for the permutation spreading technique, such as T-design and STBC design, and the parity bit selected spreading technique. Simulation results showed that the permutation spreading methods provide better BER performance compared to parity bit selected spreading technique. Moreover, results indicated that the permutation spreading using STBC design provides slightly improvement in the BER performance comparing to T-design without any increase of the system complexity.

Chapter 3 presented an overview of the SM methods. It explained how the position of 
the transmit antennas could be used in order to increase the data rate and decrease the number of RF-chains. The chapter also discussed the generalized idea of Spatial Modulation (GSM) in which more than one transmit antennas can be activated to send same data at each signaling interval. Furthermore, this chapter introduced an extension method of GSM where the number of transmit antennas to activate, at each signaling interval, varies from one transmit antenna to more than one. Simulation results showed that the performance of these three techniques is close to each other with slightly better BER performance of SM.

Chapter 4 introduced the proposed MIMO-CDMA system with permutation spreading using SM. It showed how the spatial positions of transmit antennas can be combined with permutation spreading technique in order to create an additional dependency between the data bits and the antennas combinations and thus increase the spectral efficiency of the system. The analytical expression of the BER was derived using the union bound method. Simulation and theoretical results showed that that the proposed technique provides significant improvement of the BER performance compared to the conventional STBC design and the conventional SM.

In chapter 5 , the multiusers scenario of the proposed technique was discussed. Inspired from the NOMA technique, all users were considered to use the same spreading sequences and scrambling codes that are quasi-orthogonal, were used to separate the users' signals at the receiver. Verified by simulation results, the proposed technique showed better performance compared to conventional methods. However, for high SNR values the new design had higher error floor.

\subsection{Potential future work}

The following are some suggestions for future work of this thesis:

- Investigate the BER performance of the multiuser scenario when MUD methods, such as successive interferences cancelation (SIC), are used. 
- Evaluate the performance of the proposed method under different frequency selective fading channels

- Evaluate the performance of the proposed technique in partial CSI instead of perfect CSI.

- The application of the proposed concept in OFDM systems. Similar to the SMMIMO-CDMA technique, the SM idea can be applied and parity bits can be used to select the sub-carries used to transmit the signal. 


\section{References}

[1] Ericsson. More than 50 billion connected devices. White Paper, February 2011.

[2] Andreas Molisch. Wireless communications. Wiley IEEE, Chichester, West Sussex, U.K, 2011.

[3] 3GPP TR36.913, V10.0.0. 3GPP specification: Requirements for further advancements for E-UTRA (LTE Advanced) (Release 10), Apr. 2011.

[4] Stefania Sesia. LTE - the UMTS long term evolution : from theory to practice. Wiley, Chichester, 2011.

[5] Cheng-Xiang Wang, Fourat Haider, Xiqi Gao, Xiaohu You, Yang Yang, Dongfeng Yuan, Hadi M. Aggoune, Harald Haas, S. Fletcher, and Erol Hepsaydir. Cellular architecture and key technologies for $5 \mathrm{~g}$ wireless communication networks. IEEE Communications Magazine, 52(2):122-130, 2014.

[6] Jeffrey G. Andrews, Stefano Buzzi, Wan Choi, Stephen V. Hanly, Angel E. Lozano, Anthony C. K. Soong, and Jianzhong (Charlie) Zhang. What will $5 \mathrm{~g}$ be? CoRR, abs/1405.2957, 2014.

[7] S. Wu, H. Wang, and C. H. Youn. Visible light communications for $5 \mathrm{~g}$ wireless networking systems: from fixed to mobile communications. IEEE Network, 28(6):4145, Nov 2014.

[8] F. Boccardi, R. W. Heath, A. Lozano, T. L. Marzetta, and P. Popovski. Five disruptive technology directions for 5g. IEEE Communications Magazine, 52(2):74-80, February 2014. 
[9] M. Di Renzo, H. Haas, A. Ghrayeb, S. Sugiura, and L. Hanzo. Spatial modulation for generalized mimo: Challenges, opportunities, and implementation. Proceedings of the IEEE, 102(1):56-103, Jan 2014.

[10] M. D. Renzo, H. Haas, and P. M. Grant. Spatial modulation for multiple-antenna wireless systems: a survey. IEEE Communications Magazine, 49(12):182-191, December 2011.

[11] A. Stavridis, S. Sinanovic, M. Di Renzo, H. Haas, and P. Grant. An energy saving base station employing spatial modulation. In 2012 IEEE 17th International Workshop on Computer Aided Modeling and Design of Communication Links and Networks (CAMAD), pages 231-235, Sept 2012.

[12] M. Di Renzo and H. Haas. On transmit diversity for spatial modulation mimo: Impact of spatial constellation diagram and shaping filters at the transmitter. IEEE Transactions on Vehicular Technology, 62(6):2507-2531, July 2013.

[13] M. Taherzadeh, H. Nikopour, A. Bayesteh, and H. Baligh. Scma codebook design. In 2014 IEEE 80th Vehicular Technology Conference (VTC2014-Fall), pages 1-5, Sept 2014 .

[14] M. AL-Imari, M. A. Imran, R. Tafazolli, and D. Chen. Performance evaluation of low density spreading multiple access. In 2012 8th International Wireless Communications and Mobile Computing Conference (IWCMC), pages 383-388, Aug 2012.

[15] Shanzhi Chen, Bin Ren, Qiubin Gao, Shaoli Kang, Shaohui Sun, and Kai Niu. Pattern division multiple access - a novel nonorthogonal multiple access for fifth-generation radio networks. IEEE Trans. Vehicular Technology, 66(4):3185-3196, 2017.

[16] D. Fang, Y. C. Huang, Z. Ding, G. Geraci, S. L. Shieh, and H. Claussen. Lattice partition multiple access: A new method of downlink non-orthogonal multiuser transmissions. In 2016 IEEE Global Communications Conference (GLOBECOM), pages $1-6$, Dec 2016. 
[17] Z. Ding, Y. Liu, J. Choi, Q. Sun, M. Elkashlan, C. L. I, and H. V. Poor. Application of non-orthogonal multiple access in lte and $5 \mathrm{~g}$ networks. IEEE Communications Magazine, 55(2):185-191, February 2017.

[18] 3rd Generation Partnership Project (3GPP). Study on downlink multiuser superposition transmission for LTE, Mar. 2015.

[19] Ping Yang, Yue Xiao, Bin Zhou, and Shaoqian Li. Initial performance evaluation of spatial modulation ofdm in lte-based systems. In CHINACOM, pages 102-107. IEEE Computer Society, 2011.

[20] D. Sinanović, G. Šišul, and B. Modlic. Low-papr spatial modulation for sc-fdma. IEEE Transactions on Vehicular Technology, 66(1):443-454, Jan 2017.

[21] M. Fukuma and K. Ishii. Space-time code division multiple access based on spatial modulation. In 2015 IEEE 82nd Vehicular Technology Conference (VTC2015-Fall), pages $1-5$, Sept 2015.

[22] C. D'Amours and J. Y. Chouinard. Parity bit selected and permutation spreading for cdma/mimo systems in frequency-nonselective rayleigh fading channels. In 2007 IEEE 65th Vehicular Technology Conference - VTC2007-Spring, pages 1475-1479, April 2007.

[23] BG Gopal and PG Kuppusamy. A comparative study on $4 \mathrm{~g}$ and $5 \mathrm{~g}$ technology for wireless applications. Journal of Electronics and Communication Engineering (IOSRJECE), 10(6):67-72, 2015.

[24] C. D'Amours. Parity bit selected spreading sequences: a block coding approach to spread spectrum. IEEE Communications Letters, 9(1):16-18, Jan 2005.

[25] M. Shi, C. D'Amours, and A. Yongacoglu. Design of spreading permutations for mimocdma based on space-time block codes. IEEE Communications Letters, 14(1):36-38, January 2010. 
[26] F.J. MacWilliams and N.J.A. Sloane. The Theory of Error-Correcting Codes. Northholland Publishing Company, 2nd edition, 1978.

[27] V. Tarokh, H. Jafarkhani, and A. R. Calderbank. Space-time block codes from orthogonal designs. IEEE Transactions on Information Theory, 45(5):1456-1467, Jul 1999.

[28] C. D'Amours and J. Y. Chouinard. Parity bit selected and permutation spreading for cdma/mimo systems in frequency-nonselective rayleigh fading channels. In 2007 IEEE 65th Vehicular Technology Conference - VTC2007-Spring, pages 1475-1479, April 2007.

[29] Proakis. Digital Communications 5th Edition. McGraw Hill, 2007.

[30] Raed Yousef Mesleh. Spatial modulation: a spatial multiplexing technique for efficient wireless data transmission. PhD thesis, Jacobs Uni Bremen, 2007.

[31] R. Mesleh, M. D. Renzo, H. Haas, and P. M. Grant. Trellis coded spatial modulation. IEEE Transactions on Wireless Communications, 9(7):2349-2361, July 2010.

[32] N. Serafimovski, A. Younis, R. Mesleh, P. Chambers, M. Di Renzo, C. X. Wang, P. M. Grant, M. A. Beach, and H. Haas. Practical implementation of spatial modulation. IEEE Transactions on Vehicular Technology, 62(9):4511-4523, Nov 2013.

[33] A. Younis, N. Serafimovski, R. Mesleh, and H. Haas. Generalised spatial modulation. In 2010 Conference Record of the Forty Fourth Asilomar Conference on Signals, Systems and Computers, pages 1498-1502, Nov 2010.

[34] J. Jeganathan, A. Ghrayeb, and L. Szczecinski. Generalized space shift keying modulation for mimo channels. In 2008 IEEE 19th International Symposium on Personal, Indoor and Mobile Radio Communications, pages 1-5, Sept 2008.

[35] N. Serafimovski, M. D. Renzo, S. Sinanovic, R. Y. Mesleh, and H. Haas. Fractional bit encoded spatial modulation (fbe-sm). IEEE Communications Letters, 14(5):429-431, May 2010. 
[36] A. Younis, R. Mesleh, H. Haas, and P. M. Grant. Reduced complexity sphere decoder for spatial modulation detection receivers. In 2010 IEEE Global Telecommunications Conference GLOBECOM 2010, pages 1-5, Dec 2010.

[37] J. Jeganathan, A. Ghrayeb, and L. Szczecinski. Spatial modulation: optimal detection and performance analysis. IEEE Communications Letters, 12(8):545-547, Aug 2008.

[38] Emanuele Viterbo and Joseph Boutros. A universal lattice code decoder for fading channels. IEEE Trans. Information Theory, 45(5):1639-1642, 1999.

[39] Abdelhamid Younis. Spatial modulation: theory to practice. Ph.D. dissertation, 2014.

[40] Sergio Verdú. Multiuser detection. Cambridge University Press, CambridgeK New York, 1998.

[41] Abdurazak Mudesir. Power control algorithm in cdma systems. International University Bremen, 11, 2004.

[42] S. Sriram and V. Sundararajan. Efficient pseudo-noise sequence generation for spreadspectrum applications. In IEEE Workshop on Signal Processing Systems, pages 80-86, Oct 2002 .

[43] H. Vincent Poor and Sergio Verdú. Single-user detectors for multiuser channels. IEEE Trans. Communications, 36(1):50-60, 1988. 